Prepared for the U.S. Department of Energy under Contract DE-AC05-76RL01830

\title{
Hybrid Enrichment Assay Methods for a UF 6 Cylinder Verification Station: FY10 Progress Report
}

\author{
LE Smith \\ DV Jordan \\ CR Orton \\ AC Misner \\ EK Mace
}

August 2010

Pacific Northwest

NATIONAL LABORATORY

Proudly Operated by Battelle Since 1965 


\title{
DISCLAIMER
}

This report was prepared as an account of work sponsored by an agency of the United States Government. Neither the United States Government nor any agency thereof, nor Battelle Memorial Institute, nor any of their employees, makes any warranty, express or implied, or assumes any legal liability or responsibility for the accuracy, completeness, or usefulness of any information, apparatus, product, or process disclosed, or represents that its use would not infringe privately owned rights. Reference herein to any specific commercial product, process, or service by trade name, trademark, manufacturer, or otherwise does not necessarily constitute or imply its endorsement, recommendation, or favoring by the United States Government or any agency thereof, or Battelle Memorial Institute. The views and opinions of authors expressed herein do not necessarily state or reflect those of the United States Government or any agency thereof.

\author{
PACIFIC NORTHWEST NATIONAL LABORATORY \\ operated by \\ BATTELLE \\ for the \\ UNITED STATES DEPARTMENT OF ENERGY \\ under Contract DE-AC05-76RL01830
}

Printed in the United States of America
Available to DOE and DOE contractors from the Office of Scientific and Technical Information,
P.O. Box 62, Oak Ridge, TN 37831-0062;
ph: (865) 576-8401
fax: (865) 576-5728
email: reports@adonis.osti.gov

Available to the public from the National Technical Information Service 5301 Shawnee Rd., Alexandria, VA 22312

ph: (800) 553-NTIS (6847)

email: orders@ntis.gov <http://www.ntis.gov/about/form.aspx>

Online ordering: http://www.ntis.gov 


\title{
Hybrid Enrichment Assay Methods for a UF $_{6}$ Cylinder Verification Station: FY10 Progress Report
}

\author{
LE Smith \\ DV Jordan \\ CR Orton \\ AC Misner \\ EK Mace
}

August 2010

Prepared for

the U.S. Department of Energy

under Contract DE-AC05-76RL01830

Pacific Northwest National Laboratory

Richland, Washington 99352 



\section{Summary}

Pacific Northwest National Laboratory (PNNL) is developing the concept of an automated $\mathrm{UF}_{6}$ cylinder verification station that would be located at key measurement points to positively identify each cylinder, measure its mass and enrichment, store the collected data in a secure database, and maintain continuity of knowledge on measured cylinders until the arrival of International Atomic Energy Agency (IAEA) inspectors. At the center of this unattended system is a hybrid enrichment assay technique that combines the traditional enrichment-meter method (based on the $186-\mathrm{keV}$ peak from ${ }^{235} \mathrm{U}$ ) with nontraditional neutron-induced high-energy gamma-ray signatures (spawned primarily by ${ }^{234} \mathrm{U}$ alpha emissions and ${ }^{19} \mathrm{~F}(\alpha, \mathrm{n})$ reactions). Previous work by PNNL provided proof-of-principle for the nontraditional signatures to support accurate, full-volume interrogation of the cylinder enrichment, thereby reducing the systematic uncertainties in enrichment assay due to $\mathrm{UF}_{6}$ heterogeneity and providing greater sensitivity to material substitution scenarios [Smith 2009; Smith In press].

The work described here builds on that preliminary evaluation of the non-traditional signatures, but focuses on a prototype field system utilizing $\mathrm{NaI}(\mathrm{Tl})$ and $\mathrm{LaBr}_{3}(\mathrm{Ce})$ spectrometers, and enrichment analysis algorithms that integrate the traditional and non-traditional signatures. Results for the assay of Type-30B cylinders ranging from 0.2 to $4.95 \mathrm{wt} \%{ }^{235} \mathrm{U}$, at an AREVA fuel fabrication plant in Richland, WA, are described for the following enrichment analysis methods: 1) traditional enrichment meter signature (186-keV peak) as calculated using a square-wave convolute (SWC) algorithm; 2) nontraditional high-energy gamma-ray signature that provides neutron detection without neutron detectors and 3) hybrid algorithm that merges the traditional and non-traditional signatures. Uncertainties for each method, relative to the declared enrichment for each cylinder, are calculated and compared to the uncertainties from an attended HPGe verification station at AREVA, and the IAEA's uncertainty target values for feed, tail and product cylinders. Table 1 provides a summary of those results below.

Table 1. Summary of enrichment assay results for a population of 26 Type 30B cylinders: 23 product (greater than $2.0 \mathrm{wt} \%{ }^{235} \mathrm{U}$ ), 2 natural, and 1 depleted. Results are given in terms of relative standard deviation from the declared enrichment values.

\begin{tabular}{|c|c|c|c|}
\hline Method & Product & Natural & Depleted \\
\hline Approximate Target Uncertainty [Aigner 2001] $^{1}$ & 5 & 10 & 15 \\
\hline Traditional HPGe (from AREVA) & 3.3 & 5.2 & 12 \\
\hline Traditional LaBr (SWC method) & 3.1 & 9.4 & 11 \\
\hline Traditional NaI (SWC method) & 3.5 & 7.9 & 13 \\
\hline Non-Traditional NaI & 3.7 & 4.9 & 32 \\
\hline Hybrid NaI (simple average) & 2.5 & 4.6 & 9.7 \\
\hline
\end{tabular}

${ }^{1}$ The target uncertainties are estimated from the combination of systematic and random errors given in Kuhn and should be used only to set the scale of the target uncertainties. 
A summary of the major findings from the field measurements and subsequent analysis follows:

- Traditional enrichment-meter assay using specially collimated NaI spectrometers and a SquareWave-Convolute algorithm can achieve uncertainties comparable to HPGe and $\mathrm{LaBr}$ for product, natural and depleted cylinders.

- Non-traditional signatures measured using $\mathrm{NaI}$ spectrometers enable interrogation of the entire cylinder volume and accurate measurement of absolute ${ }^{235} \mathrm{U}$ mass in product, natural and depleted cylinders.

- A hybrid enrichment assay method can achieve lower uncertainties than either the traditional or non-traditional methods acting independently because there is a low degree of correlation in the systematic errors of the two individual methods (wall thickness variation and ${ }^{234} \mathrm{U} /{ }^{235} \mathrm{U}$ variation, respectively).

This work has indicated that the hybrid NDA method has the potential to serve as the foundation for an unattended cylinder verification station. When compared to today's handheld cylinder-verification approach, such a station would have the following advantages: 1) improved enrichment assay accuracy for product, tail and feed cylinders; 2) full-volume assay of absolute ${ }^{235} \mathrm{U}$ mass; 3) assay of minor isotopes $\left({ }^{234} \mathrm{U}\right.$ and $\left.{ }^{232} \mathrm{U}\right)$ important to verification of feedstock origin; single instrumentation design for both Type 30B and Type 48 cylinders; and 4) substantial reduction in the inspector manpower associated with cylinder verification.

The proof-of-principle results presented in this report encourage further study of the strengths and limitations of the hybrid method, and comparison to other NDA methods being proposed for automated cylinder verification stations. The major tasks proposed for FY11 and FY12 investigation are:

Refine and Validate Modeling Framework: High-fidelity modeling of the $\mathrm{UF}_{6}$ cylinder scenario is a time- and resource-efficient complement to other development tasks, and aids the exploration of systematic errors that are difficult to assess with field data (e.g., the effect of $\mathrm{UF}_{6}$ spatial distribution variations). PNNL developed a functional and preliminarily validated modeling framework in FY10, tools that will be further expanded, refined and validated.

Perform System Design Parameter Study: There are a number of system design choices to be considered in a hybrid assay method instrument, including detector size, orientation and collimator/converter designs. Modeling will be used to explore different system designs that strike a balance between the performance of the traditional assay method (which benefits from a high degree of collimation and small detector area) and the non-traditional method (which benefits from less collimation and larger detectors).

Refine and Evaluate Traditional Method using Square-Wave Convolute Algorithm: The Square Wave Convolute (SWC) algorithm developed and implemented in the FY10 study, though first-generation, was effective. We will develop and test extension of the SWC to accurately remove the nonlinear continuum contributions, then compare it to other codes to assess the relative strengths and weaknesses in terms of assay accuracy and practical operational issues such as robustness against gain and resolution instabilities.

Perform Additional Field Measurements and Compare to Other NDA Approaches: The FY10 study indicated that a hybrid assay method can meet target uncertainties for depleted and natural enrichments, but that analysis was based on very few Type 30B cylinders, rather than the Type 48 cylinders that are the 
industry standard for feed and tail material. To definitively assess the viability of the hybrid NDA methods for product, tail and feed cylinders will require an extended measurement campaign at an operating enrichment plant. PNNL will pursue collaboration with Los Alamos National Laboratory (LANL) for such a campaign, with the goal of side-by-side operation with LANL's Passive Neutron Enrichment Meter (PNEM) that utilizes totals and coincidence neutron counting to achieve NDA objectives similar to those achieved by PNNL's hybrid methods. A comparative evaluation of the two methods, against the same cylinder population, will provide insights into their strengths and limitations in terms of accuracy and operational practicality, and help inform further development of both methods as appropriate. 



\section{Acknowledgments}

Funding for this work was provided by the U.S. National Nuclear Security Administration's (NNSA) Office of Nonproliferation and International Security (NA-24) and the Next Generation Safeguards Initiative. The authors are appreciative to NA-24's Kevin Veal and Steve Lamontagne for their support of this work and helpful insights, both programmatic and technical. The authors would also like to express their appreciation to AREVA NP, Inc., Richland, WA, USA, and Dan Noss and Herbert Ford for their support of the cylinder measurements. Finally, we thank Kevin Anderson, Mark Shaver and Michael Curtis of Pacific Northwest National Laboratory for their assistance in the statistical analysis of the field data, MCNP modeling, and guidance on enrichment-plant safeguards practices, respectively. 



\section{Acronyms and Abbreviations}

\begin{tabular}{|c|c|}
\hline ES & Environmental sampling \\
\hline FWHM & Full width at half maximum \\
\hline GCEP & Gaseous centrifuge enrichment plant \\
\hline HEU & Highly enriched uranium \\
\hline HPGe & High-purity germanium \\
\hline IAEA & International Atomic Energy Agency \\
\hline ICVS & Integrated cylinder verification station \\
\hline INMM & Institute of Nuclear Materials Management \\
\hline LANL & Los Alamos National Laboratory \\
\hline MCNP & Monte Carlo N- Particle \\
\hline NA-24 & Office of Nonproliferation and International Security \\
\hline NDA & Non-destructive assay \\
\hline NNSA & National Nuclear Security Administration \\
\hline ORNL & Oak Ridge National Laboratory \\
\hline PNEM & Passive neutron enrichment meter \\
\hline PNNL & Pacific Northwest National Laboratory \\
\hline SWC & Square wave convolute \\
\hline USEC & United States Enrichment Corporation \\
\hline
\end{tabular}





\section{Contents}

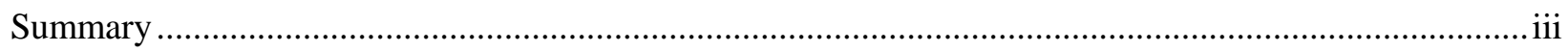

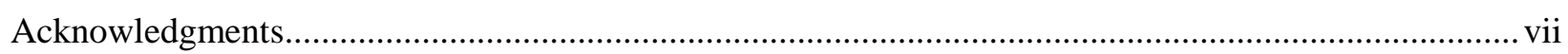

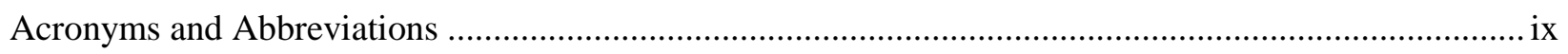

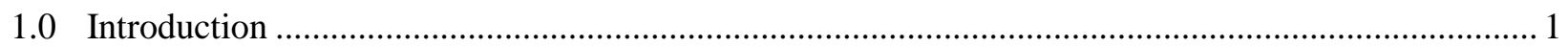

2.0 Signatures and Algorithms for Enrichment Assay ….................................................................... 3

2.1 Traditional Enrichment Meter Method using 186-keV Peak as Direct Measure of ${ }^{235} \mathrm{U}$............. 3

2.1.1 Peak Area Extraction: SWC Algorithm Implementation .......................................4

2.1.2 Peak Area Extraction: Initial Testing of SWC Algorithm.......................................8

2.1.3 Application of the SWC to the Enrichment Meter Algorithm ..............................10

2.2 Non-Traditional High-Energy Gamma Rays as Indirect Measure of ${ }^{235} \mathrm{U}$............................... 11

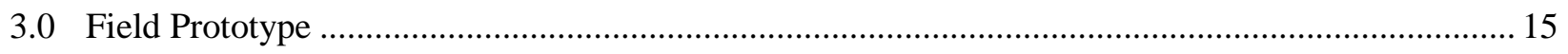

3.1 Calibration and Characterization of Gamma-Ray Spectrometers .......................................... 15

3.2 Collimation and Neutron-to-Gamma Converters .................................................................... 19

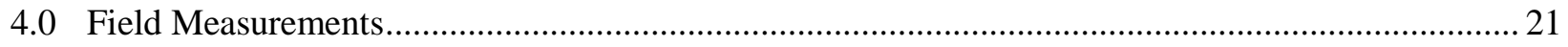

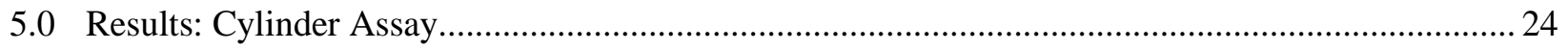

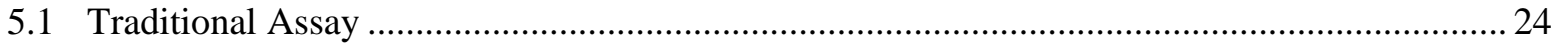

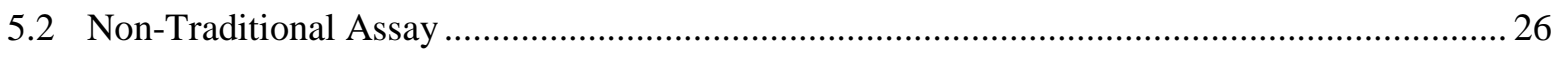

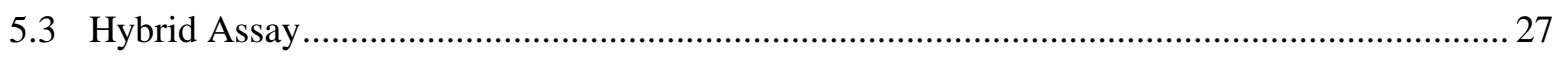

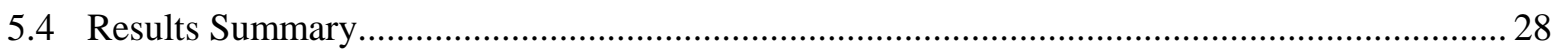

6.0 Interim Conclusions: Viability of Hybrid Assay Methods ............................................................ 31

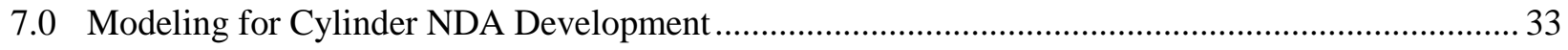

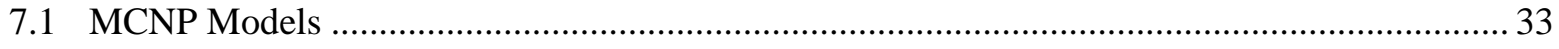

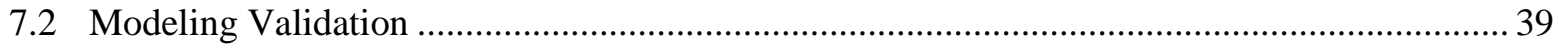

8.0 Case Study: Non-Traditional Signature for Reactor-Recycle Feedstock ....................................... 42

8.1 ORIGEN-ARP for Modeling of Reactor-Recycle Uranium Isotopics .................................... 42

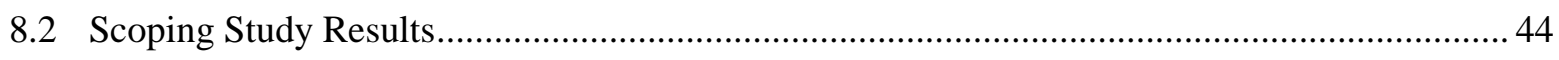

8.2.1 234/235 Variation in Reactor-Recycle Uranium...............................................44

8.2.2 Uranium-232 as Quantitative Indicator of 234/235 ...........................................47

8.3 Preliminary Findings: Non-Traditional Signature for Reactor-Recycle Uranium .................... 52

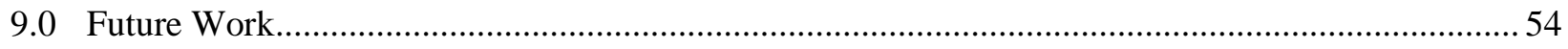

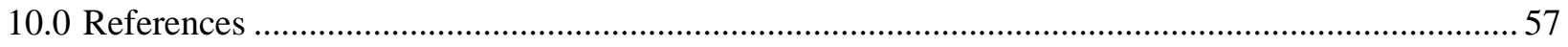




\section{Figures}

Figure 1. Conceptual design of an integrated $U_{6}$ cylinder verification station (ICVS) that includes a cylinder trolley, mass measurement using a floor scale, cylinder identification, and hybrid uranium enrichment methods using medium-resolution gamma-ray spectrometers (in blue panels)

Figure 2. Illustration of the square-wave convolute method on two $\mathrm{UF}_{6}$ spectra recorded from 30B cylinders with a $\mathrm{NaI}(\mathrm{Tl})$ spectrometer. The SWC effectively removes the constant and linear components of the underlying continuum.

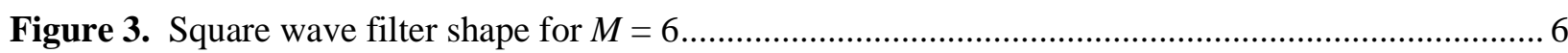

Figure 4. Illustration of effect of square-wave filter on a simple "toy" spectrum consisting of a Gaussian peak superimposed on a linear background .....

Figure 5. Calculated neutron production rate in a Type $30 \mathrm{~B}$ cylinder. The ${ }^{234} \mathrm{U}$ is the dominant source term above approximately $0.5 \mathrm{wt} \%{ }^{235} \mathrm{U}$. The ${ }^{238} \mathrm{U}(\alpha, \mathrm{n})$ and spontaneous fission source terms are approximately equal over all enrichments and are summed in this figure. We ignored uranium-235 contributions here since they are comparatively insignificant.

Figure 6. Spectra recorded by two large $\mathrm{NaI}(\mathrm{Tl})$ spectrometers and two HPGe spectrometers from a 30B cylinder enriched to $4.95 \%$. The higher interaction efficiency of the $\mathrm{NaI}(\mathrm{Tl})$ enables the collection of neutron-induced high-energy signatures. In this work, that signature is defined with a window between 3.0 and $8.0 \mathrm{MeV}$ (yellow box).

Figure 7. Energy resolution for the $\mathrm{LaBr}$ and $\mathrm{NaI}(7.6 \times 7.6 \mathrm{~cm})$ spectrometers as a function of bipolar shaping time and high-voltage (top) and incident energy (bottom) at appropriate shaping and high-voltage settings.....

Figure 8. Top: Observed count rate as a function of the incident count rate. Bottom: Energy resolution at $662-\mathrm{keV}$ as a function of incident count rate.

Figure 9. Left: Field demonstration prototype that includes collimated three $8 \times 3.8 \mathrm{~cm} \mathrm{LaBr}$ and seven $6 \times 7.6 \mathrm{~cm} \mathrm{NaI}$ spectrometers, and a $5.1 \times 10.2 \times 20.3 \mathrm{~cm} \mathrm{NaI}$ (middle detector) wrapped in layers of iron and polyethylene. Right: Lead (top) and Fe/poly (bottom) collimator designs used for the $\mathrm{LaBr}$ and $7.6 \times 7.6 \mathrm{~cm} \mathrm{NaI}$ spectrometers. The Fe/poly cylindrical collimator (with lead endcap aperture) is intended to enhance the high-energy gamma-ray signature.

Figure 10. Photographs of experimental setup at AREVA NP fuel fabrication facility ...

Figure 11. Example spectra recorded from a 30B cylinder enriched to $4.4 \mathrm{wt} \%{ }^{235} \mathrm{U}$. Left: $3.8 \times$ $3.8 \mathrm{~cm} \mathrm{LaBr}$ and $7.6 \times 7.6 \mathrm{~cm} \mathrm{NaI}$ for energies to $1.2 \mathrm{MeV}$. Right: $5 \times 10 \times 20 \mathrm{~cm} \mathrm{NaI}$ for energies to approximately $8 \mathrm{MeV}$.

Figure 12. Assay enrichment versus declared enrichment using the traditional enrichment-meter method and a square-wave convolute algorithm for the calculation of net count rate in the 186-keV peak: $\mathrm{LaBr}$ (Top) and $\mathrm{NaI}$ (Bottom). The analysis data set spans 26 full cylinders ( 0.2 to $5 \mathrm{wt} \%{ }^{235} \mathrm{U}$ ) but does not include the HEU down-blend cylinders (red data points). The one-sigma error bars reflect only the statistical counting uncertainty in the assay enrichment. 
Figure 13. Assay values for ${ }^{235} \mathrm{U}$ mass versus declared ${ }^{235} \mathrm{U}$ mass using the non-traditional highenergy gamma-ray signature. HEU downblend cylinders are plotted but are not included in the uncertainty estimates: $3.7 \%$ for product cylinders and $4.9 \%$ for natural enrichment. The non-traditional signature interrogates the entire cylinder volume and assays absolute ${ }^{235} \mathrm{U}$ mass, rather than a localized, relative enrichment level.

Figure 14. Comparison of non-traditional (high-energy gamma) and traditional (186-keV) assay of 26 cylinders over a range of ${ }^{235} \mathrm{U}$ enrichment. The standard deviations of the nontraditional and traditional assay of product cylinders are 3.7\% and 3.5\%, respectively.

Figure 15. Photograph of AREVA's attended cylinder verification station, consisting of a wellcollimated HPGe spectrometer in a fixed gantry geometry

Figure 16. Cross-section of MCNP5 geometry used in the simulation of field measurements at the AREVA fuel fabrication plant in April and May of 2010.

Figure 17. Measured sensor energy resolution data and fits to the data for $\mathrm{UF}_{6}$ cylinder assay portal deployed at the AREVA fuel fabrication plant in April and May 2010.

Figure 18. Schematic of the MCNP5 modeling approach used to estimate an infinite thickness for gamma-energies of 186, 1001 and $2614 \mathrm{keV}$

Figure 19. Example of data from infinite-thickness simulations for the $1001 \mathrm{keV}$ emitted by the ${ }^{238} \mathrm{U}$ daughter ${ }^{234 \mathrm{~m}} \mathrm{~Pa}$. Based on this data, one can approximate the gamma-ray flux at the surface of a cylinder filled to capacity with $\mathrm{UF}_{6}$ using a $10-\mathrm{cm}$ cylindrical shell

Figure 20. Schematic of the shell geometry used to reduce the variance in MCNP modeling of the $\mathrm{UF}_{6}$ cylinder assay scenario. The MCNP tally results were normalized using a volumetric mass correction utilizing the parameters $\mathrm{x}, \mathrm{r}$ and $\mathrm{R}$

Figure 21. Examples of basis vectors simulated using MCNP5, and their summation to produce a prediction for the spectrum measured by the prototype field system. Top: 7.6 $\times$ 7.6-cm NaI. Bottom: LaBr. Black trace: Sum, 4.4\% ${ }^{235} \mathrm{U}, 200$ ppt ${ }^{232} \mathrm{U}$. Red: Uranium-235 (4.4\%). Green: Uranium-238 (95.6\%). Blue: Uranium-232 (200 ppt). Magenta: Uranium-234 ( $\alpha, n)$. Brown: Uranium-238 spontaneous fission.

Figure 22. Examples of basis vectors for the $5 \times 10 \times 20 \mathrm{~cm} \mathrm{NaI}$ spectrometer simulated using MCNP5, and their summation to produce a prediction for the spectrum measured by the prototype field system.

Figure 23. Comparison of measured (black curves) and simulated (red curves) spectra for the assay of cylinders of various enrichments with the $\mathrm{LaBr}$ (left) and 7.6 × 7.6-cm NaI (right) spectrometers. Top: Natural (0.711 wt $\left.\%{ }^{235} \mathrm{U}\right)$. Middle: $2.0 \mathrm{wt} \%{ }^{235} \mathrm{U}$. Bottom: $4.4 \mathrm{wt} \%$ ${ }^{235} \mathrm{U}$

Figure 24. Comparison of measurements and simulations of the non-traditional high-energy gamma-ray signature (3.0 to $8.0 \mathrm{MeV}$ ) for a range of cylinder enrichments.

Figure 25. Select production and loss mechanisms for ${ }^{234} \mathrm{U}$ within a light-water reactor.

Figure 26. Uranium-234/Uranium-235 ratio for PWR fuel with an initial enrichment of 2 wt\%

${ }^{235} \mathrm{U}$, as a function of burnup and cooling time

Figure 27. Uranium-234/Uranium-235 ratio for PWR fuel with an initial enrichment of $5 \mathrm{wt} \%$ ${ }^{235} \mathrm{U}$, as a function of burnup and cooling time 45 
Figure 28. Uranium-234/Uranium-235 ratio for PWR fuel with a cooling time of 3 years, as a function of initial enrichment and burnup

Figure 29. Uranium-234/Uranium-235 variation for PWR fuel over the range of initial enrichment, burnup and cooling time considered in this study. The maximum 234/235 value is approximately 30 times higher than the naturally occurring ratio.

Figure 30. Production and loss pathways of ${ }^{232} \mathrm{U}$ in light-water reactor fuel [Cochran 1990].

Figure 31. Gamma-ray spectra recorded from three $\mathrm{UF}_{6}$ cylinders, two of which are from orebased feed (red and green) and the third from HEU downblend activities in Russia (blue). The presence of ${ }^{232} \mathrm{U}$ in the reactor-recycle uranium is evident from the 2614 -keV gammaray emitted by ${ }^{208} \mathrm{Tl}$, a daughter of ${ }^{232} \mathrm{U}$.

Figure 32. Uranium-232/( $\left.{ }^{234} U /{ }^{235} U\right)$ for a cooling time of 3 years, as a function of initial enrichment and burnup

Figure 33. Uranium-232/( $\left.{ }^{234} \mathrm{U} /{ }^{235} \mathrm{U}\right)$ for a burnup of $45 \mathrm{GWd} / \mathrm{MTU}$, as a function of initial enrichment and burnup

Figure 34. Uranium-232/( $\left.{ }^{234} U /{ }^{235} U\right)$ for an initial enrichment of $4 \mathrm{wt} \%{ }^{235} \mathrm{U}$, as a function of initial enrichment and burnup 51

Figure 35. Plot of ${ }^{232} U /\left({ }^{234} U /{ }^{235} U\right)$ ratio for all burnup levels, cooling times and ${ }^{235} \mathrm{U}$ enrichments......... 51

Figure 36. ORIGEN-ARP data for the behavior of ${ }^{232} \mathrm{U}$ and ${ }^{236} \mathrm{Pu}$ in spent fuel based on 1 metric ton of $4 \%{ }^{235} \mathrm{U}$ enriched uranium and 45 GWD/MTU burnup

\section{Tables}

Table 1. Summary of enrichment assay results for a population of 26 Type 30B cylinders: 23 product (greater than $2.0 \mathrm{wt} \%{ }^{235} \mathrm{U}$ ), 2 natural, and 1 depleted. Results are given in terms of relative standard deviation from the declared enrichment values

Table 2. Summary of enrichment assay results for a population of 26 Type 30B cylinders: 23 product (greater than $2.0 \mathrm{wt} \%{ }^{235} \mathrm{U}$ ), 2 natural, and 1 depleted. Results are given in terms of relative standard deviation from the declared enrichment values.

Table 3. Parameters for the MCNP GEB resolution function used in modeling the energy response of the three spectrometers deployed at AREVA in April and May 2010. We determined the parameters by fitting measured energy resolution data.

Table 4. Input parameters for ORIGEN_ARP Express modeling of PWR fuel assemblies

Table 5. Matrix of PWR fuel parameters considered in the ORIGEN study of 234/235 variation... 44

Table 6. Uranium isotopic masses (grams) in the ORIGEN-ARP fuel definitions, as a function of initial uranium enrichment. 
PNNL-19854

\subsection{Introduction}

International Atomic Energy Agency (IAEA) inspectors currently perform periodic inspections at uranium enrichment plants to verify $\mathrm{UF}_{6}$ cylinder enrichment declarations. Measurements are typically performed with handheld high-resolution sensors on a sampling of cylinders taken to be representative of the facility's entire cylinder inventory. There are recognized shortcomings with the current cylinder verification approach. The first is that the $186-\mathrm{keV}$ gamma ray used in the traditional enrichment meter approach is weakly penetrating and therefore, assays only the outer 1 to $2 \mathrm{~cm}$ of the $\mathrm{UF}_{6}$ volume; when combined with a small field of view for the collimated instrument, the result is a highly localized assayjust a few $\mathrm{cm}^{3}(<0.1 \%)$ of the entire cylinder. For cylinders that are not well-homogenized, either from legitimate process variation (particularly common for tails cylinders), or through diversion scenarios (e.g., substitution of highly enriched material), this localized assay can be problematic. Another shortcoming of the current method is that local variations in the wall thickness can lead to large systematic uncertainties in the 186-keV peak intensity. This means that cylinder thickness measurements are required to make attenuation corrections, another manpower-intensive activity for inspectors.

Pacific Northwest National Laboratory (PNNL) is developing an Integrated Cylinder Verification Station (ICVS) approach that is intended to support $100 \%$ cylinder verification (as opposed to samplingbased verification during periodic inspections of an enrichment facility) and to provide volume-averaged cylinder enrichment assay, and do both while reducing inspector manpower needs. The ICVS concept (Figure 1) includes the use of a cylinder trolley that traverses a verification station consisting of mass measurement (using a floor scale), cylinder identification (e.g., the IAEA has developed and is testing a laser-based system), and preservation of continuity of knowledge (e.g., using the IAEA's digital-camerabased technologies). A full description of the ancillary systems and approaches being proposed for the ICVS is presented in Supplemental Systems for Unattended $U_{6}$ Cylinder Monitoring [Curtis In Press]. 


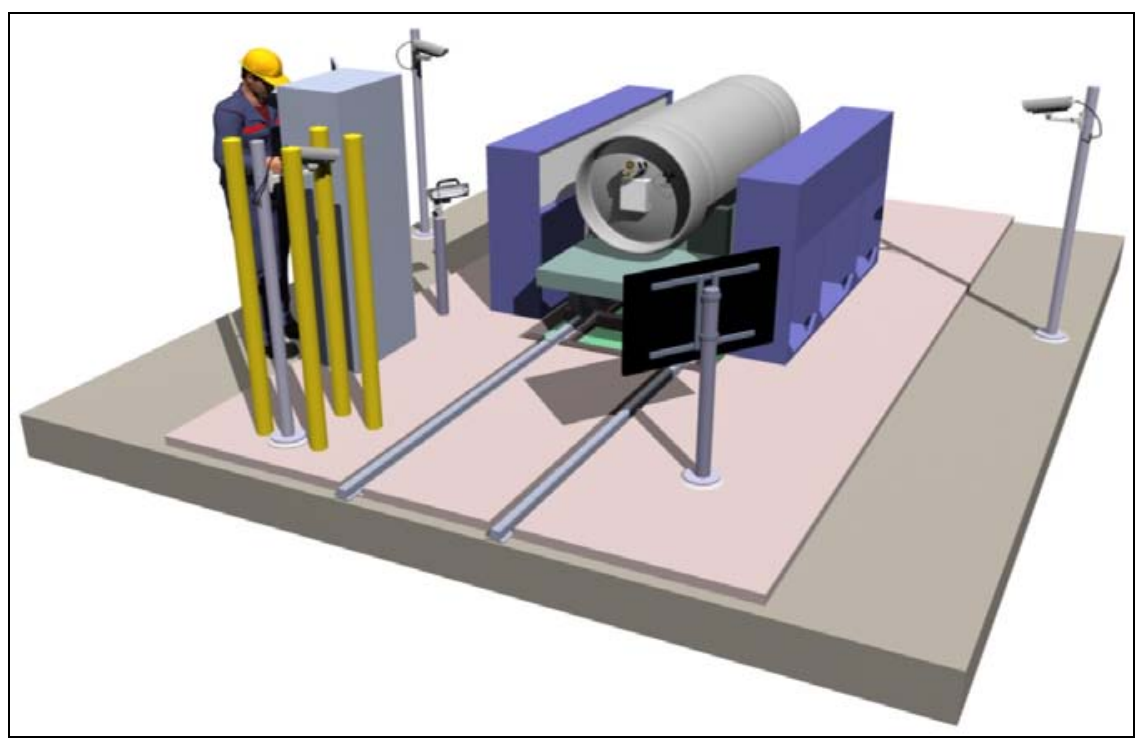

Figure 1. Conceptual design of an integrated $\mathrm{UF}_{6}$ cylinder verification station (ICVS) that includes a cylinder trolley, mass measurement using a floor scale, cylinder identification, and hybrid uranium enrichment methods using medium-resolution gamma-ray spectrometers (in blue panels)

In order to maximize the effectiveness of the ICVS's full volume analysis, advanced NDA assay methods are necessary such as the new hybrid NDA methods presented below. The primary technical question addressed in this report is whether a cylinder NDA method that merges the traditional 186-keV signature with more-penetrating non-traditional signatures can meet the target uncertainties specified by the IAEA [Aigner 2001]. The research presented here compares and contrasts the strengths and limitations of this new hybrid NDA method to the handheld-based verification methods used today; we will discuss this in the context of modern centrifuge enrichment plants that process feed, tail and product cylinders. This report suggests next steps in the exploration of hybrid NDA methods. 


\subsection{Signatures and Algorithms for Enrichment Assay}

We are currently considering two signatures in the development of NDA methods for the ICVS: the traditional 186-keV gamma ray emitted in ${ }^{235} \mathrm{U}$ decay, and non-traditional high-energy gamma rays induced by neutron interactions. We describe each below in a section that draws heavily from Eric Smith's IEEE conference paper Signatures and Methods for the Automated Nondestructive Assay of UF 6 [In press].

\subsection{Traditional Enrichment Meter Method using 186-keV Peak as Direct Measure of ${ }^{235} \mathrm{U}$}

The traditional 186-keV analysis provides a direct measure of ${ }^{235} \mathrm{U}$. While the resolution of mediumresolution scintillators (e.g., $\mathrm{NaI}(\mathrm{Tl})$ and $\mathrm{LaBr}_{3}(\mathrm{Ce})$ ) is inferior to that of high-purity germanium (HPGe), it is still adequate to accurately quantify the net number of counts in the $186-\mathrm{keV}$ peak-if we can realize sufficiently robust methods for subtracting a widely varying continuum. For example, previous work by other groups has shown that NaI-based 186-keV peak analysis, using a collimated handheld device and measurements at a single location on the cylinder, can achieve approximately 5\% uncertainty for product cylinders [Walton 1974; Dias 2008]. An underlying assumption of this work is that a fixed-geometry instrument that utilizes an array of medium-resolution scintillator spectrometers can reduce systematic uncertainties due to background variations and $\mathrm{UF}_{6}$ heterogeneity in the cylinder. If this assumption is proven valid, it may be possible to realize assay uncertainty of less than the $5 \%$ reported in previous product-cylinder measurements as well as improve assay accuracy for natural and depleted cylinders.

A number of possible algorithms exist for determining the net area of the $186-\mathrm{keV}$ peak. In this work, a zero-area square-wave digital filter [Janssens 1991] is applied to the raw energy-deposition spectrum of counts versus channel. The convolution of the original spectrum with the filter is referred to as the "square wave convolute" (SWC) spectrum. A key advantage of a symmetric zero-area digital filter is that by construction, it removes background components varying linearly with energy. Application of the filter to a symmetric peak superimposed on a linear background yields a characteristic shape in the SWC spectrum. The central portion of this shape is a quasi-Gaussian peak with (a) essentially the same position as the original peak, and (b) amplitude proportional to the area of the original peak. For a Gaussian peak in the original spectrum with standard deviation $\sigma$, the intensity enhancement in the SWC peak amplitude relative to the original peak amplitude is $\approx \sqrt{2 \pi} \sigma$. Non-zero curvature in the shape of the underlying continuum will distort this simple proportionality between SWC amplitude and original peak area, but the relationship is still linear in the presence of a quadratic background component [Janssens 1991]. Although a zero-area digital filter is typically used for peak recognition and interference detection, Opdebeeck documents efforts to extract peak area information from the amplitude of the SWC peak [1975]. In the present application, the SWC technique offers a potentially attractive alternative to nonlinear fitting (i.e., Gaussian + polynomial background) of the original spectrum: The location of the 186-keV peak in the SWC spectrum is easily determined by pattern recognition (see for example Figure 2), so that the method can be robust against spectrometer gain drift. 


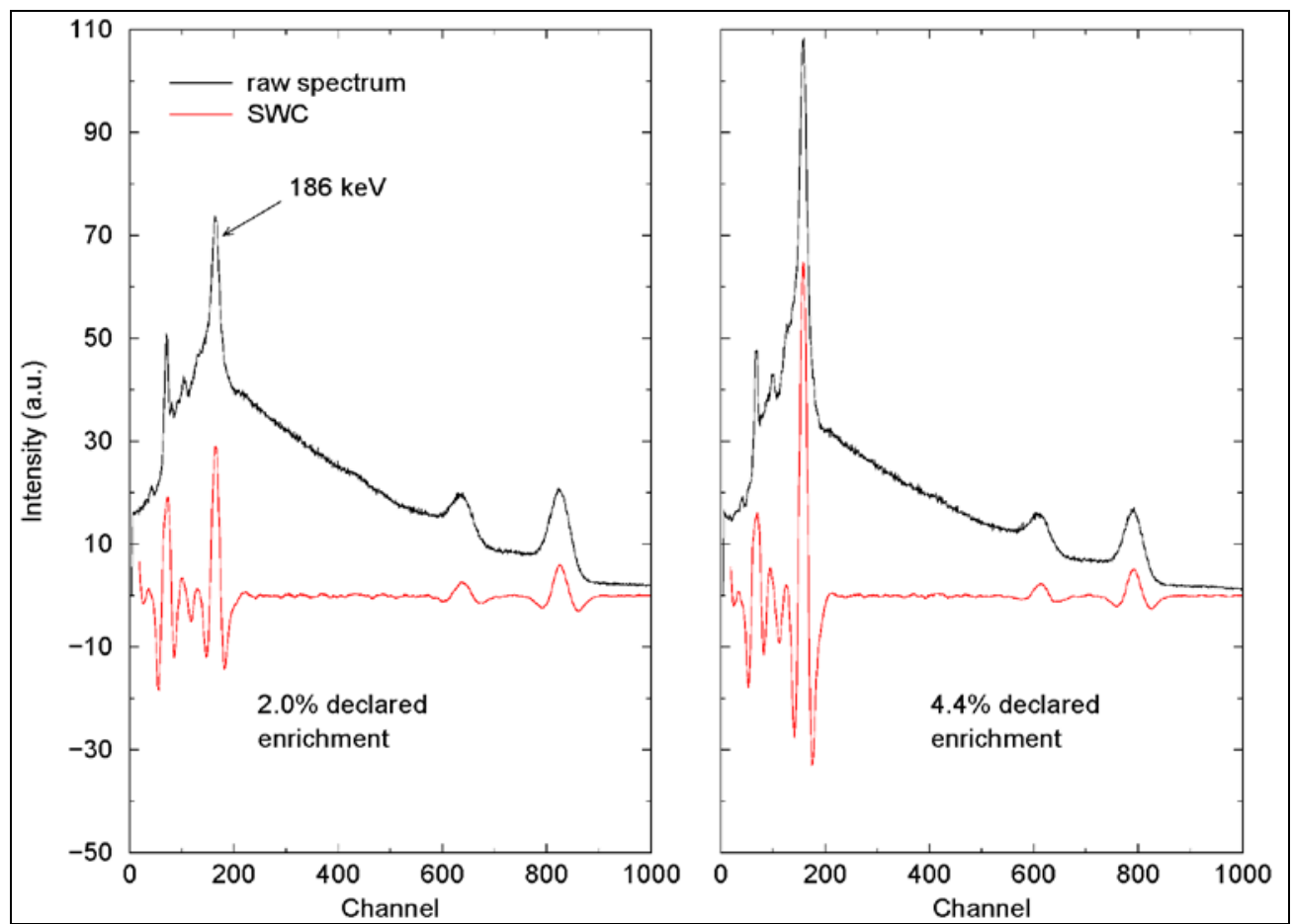

Figure 2. Illustration of the square-wave convolute method on two $\mathrm{UF}_{6}$ spectra recorded from $30 \mathrm{~B}$ cylinders with a $\mathrm{NaI}(\mathrm{Tl})$ spectrometer. The SWC effectively removes the constant and linear components of the underlying continuum.

\subsubsection{Peak Area Extraction: SWC Algorithm Implementation}

The peak area extraction algorithm adopted for the current work involves application of a discrete form of the so-called square-wave filter [Opdebeeck 1975; Janssens 1991] to the pulse-height spectra collected with the $\mathrm{NaI}(\mathrm{Tl})$ or $\mathrm{LaBr}_{3}(\mathrm{Ce})$ spectrometers. Figure 3 illustrates the shape of the digital filter, $g(t)$, which is fully described by a single width parameter, $M$. The variable $t$ (and, similarly, $\tau$ in the convolute spectrum discussed below) labels channel number in the pulse-height spectrum. The filter is a symmetric function of $t$ and has zero total area. In its continuous form, the filter can be described via

$$
\begin{aligned}
g(t) & =-1, \quad-M \leq t<-M / 2 \\
& =1, \quad-M / 2 \leq t \leq M / 2 \\
& =-1, \quad M / 2<t \leq M
\end{aligned}
$$

and vanishes otherwise. Application of the filter to the pulse-height spectrum, $f(t)$, then takes the form

$$
\begin{aligned}
R(\tau) & =\int_{\tau-M}^{\tau+M} f(t) g(\tau-t) d t \\
& =-\int_{\tau-M}^{\tau-M / 2} f(t) d t+\int_{\tau-M / 2}^{\tau+M / 2} f(t) d t-\int_{\tau+M / 2}^{\tau+M} f(t) d t
\end{aligned}
$$


PNNL-19854

where $R(\tau)$ is the convolute spectrum.

In an actual pulse-height spectrum, the channel index, $t$, is a discrete variable. It is convenient to generalize slightly the convolute definition in order to account for sampling of a finite number of discrete channels. (In essence, we must take into account the fact that discontinuities in the discrete filter shape occur over a finite distance in terms of the continuous spectrum variable, $t$, in contrast to the infinitely sharp discontinuities defined in Eq. (1).) Starting with the continuous-variable filter function $g(t)$ and restricting $M$ to integer values, we define

$$
A=\frac{\sum_{t=-M}^{M} g(t)}{2 M+1}
$$

for $M$ either even or odd, and the discrete-variable filter function is then defined via

$$
\tilde{g}(t) \equiv g(t)-A
$$

Note that by construction the modified filter satisfies

$$
\sum_{t=-M}^{M} \tilde{g}(t)=0
$$

i.e., it is a "zero-area" (or, more precisely, a zero-sum) filter for the discrete channel set $\{-M,-M+1, \ldots$, $M-1, M$. The convolute $R(\tau)$ in the discrete case is then defined in a manner analogous to (2) for the continuous case,

$$
R(\tau)=\sum_{t=\tau-M}^{\tau+M} f(t) \tilde{g}(\tau-t)
$$

We note in passing that (6) amounts to a linear combination of the contents of $2 M+1$ original pulseheight spectrum channels. The standard deviation of the convolute in bin $\tau$ is thus readily computed in terms of the standard deviations of the original pulse-height spectrum's channel contents via

$$
\sigma_{R}(\tau)=\sqrt{\sum_{t=\tau-M}^{\tau+M} \sigma_{f}^{2}(t) \tilde{g}^{2}(\tau-t)} .
$$


PNNL-19854

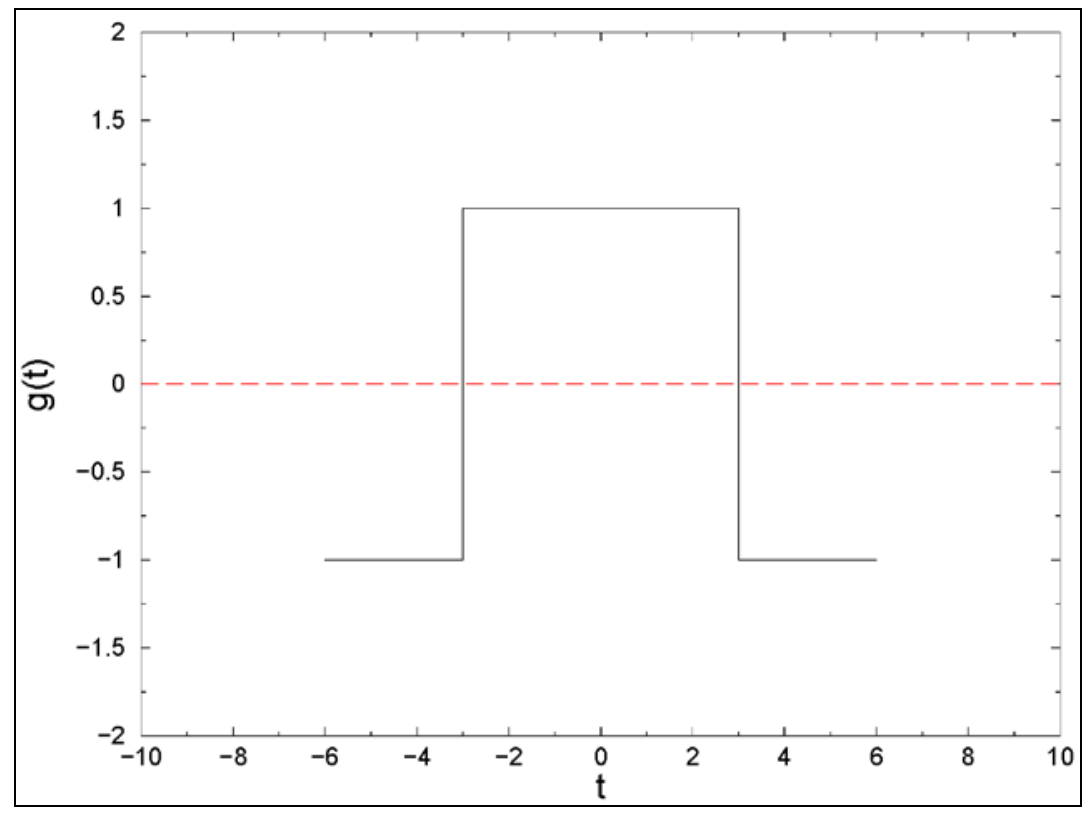

Figure 3. Square wave filter shape for $M=6$

Figure 4 illustrates the effect of applying the discrete square-wave filter defined in Eq. (4) to a simple "toy" spectrum consisting of a pure Gaussian peak superimposed on a linear background. This example clearly expresses key qualitative features of the convolute spectrum :

- The linearly-varying background portion of the raw spectrum was effectively filtered out, yielding a flat baseline.

- A characteristic positive-going central peak plus negative-going side "troughs" has replaced the Gaussian peak. The position of the central peak of the convolute spectrum essentially coincides with the position of the Gaussian in the original spectrum. The positions of the two side-troughs roughly demarcate the points in the original spectrum at which the Gaussian peak returns to the level of the linear background component. 
PNNL-19854

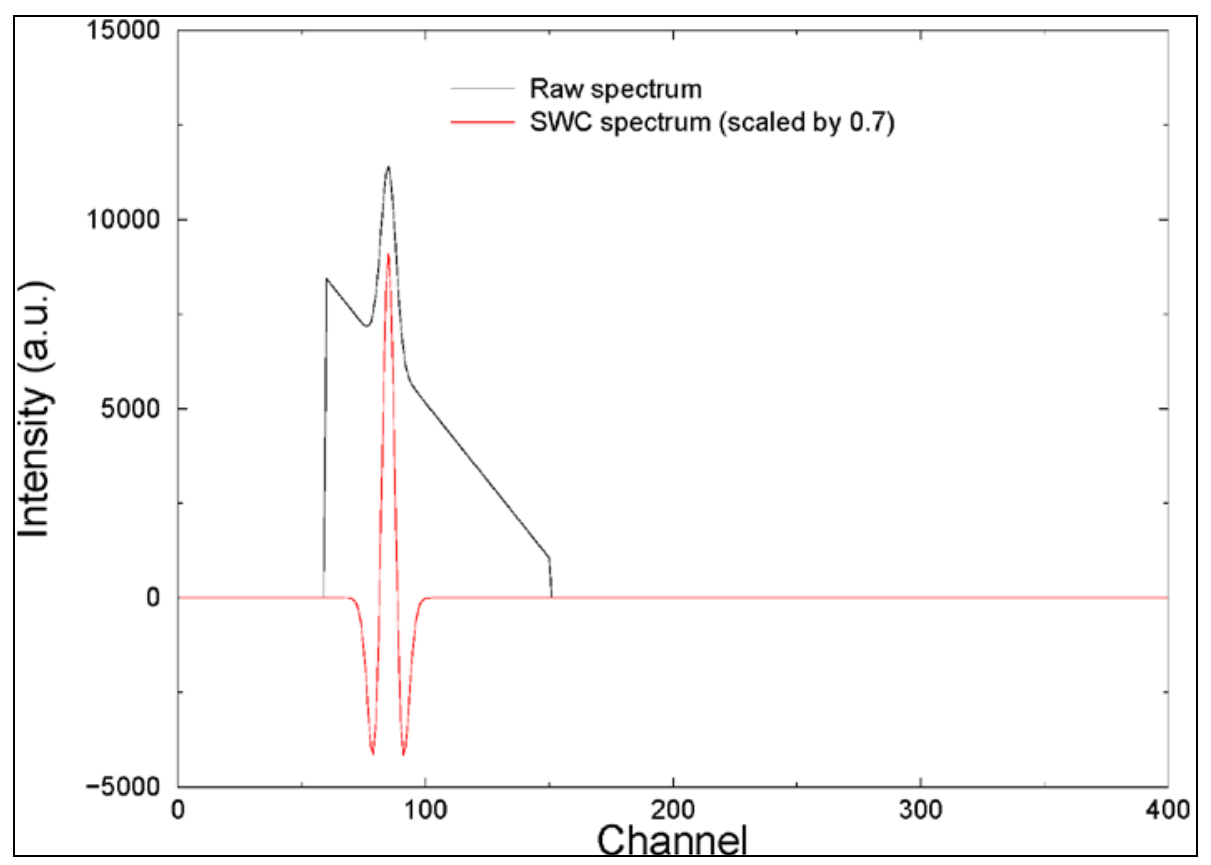

Figure 4. Illustration of effect of square-wave filter on a simple "toy" spectrum consisting of a Gaussian peak superimposed on a linear background

An important property of the square-wave filter for the purposes of the current work is the correlation between the amplitude of the convolute peak and the area of the (Gaussian) peak in the original spectrum. Adopting a slightly modified form of the notation in Janssens [1991], we denote the Gaussian "signal" peak by $S(t)$, the background continuum by $B(t)$, and the total pulse-height spectrum by $D(t)$ :

$$
\begin{aligned}
& D(t)=S(t)+B(t) \\
& =I_{0} \exp \left[-\frac{\left(t-t_{0}\right)^{2}}{2 \sigma^{2}}\right]+b_{0}+b_{1} t+b_{2} t^{2}
\end{aligned}
$$

where $I_{0}, t_{0}$, and $\sigma$ refer to the amplitude, position, and standard deviation of the Gaussian peak, respectively, and the background (assumed to have at most a quadratic dependence on channel number) is described by a polynomial with coefficients $b_{i}, I=0,1,2$. Janssens [1991] shows that for the continuous version of the square-wave filter (as defined in Eq. (1) and (2)), the convolute spectrum of $D(t)$ takes the form

$$
R(\tau)=\sqrt{2 \pi} I_{0} \sigma F\left(\tau ; t_{0}, \sigma, M\right)-\frac{1}{2} b_{2} M^{3}
$$

where the function $F$ can be expressed analytically in terms of a sum of error functions. $F$ is independent of the amplitude, $I_{0}$, of the Gaussian peak and reaches its peak value at $\tau=t_{0}$, showing that (in the continuous case, at least) the position of the convolute peak coincides with the position of the original Gaussian peak. Comparing (9) to the area (i.e., total counts) of the Gaussian peak,

$$
C_{\text {tot }}=\sqrt{2 \pi} I_{0} \sigma
$$

we find that the amplitude of the convolute peak, $R\left(t_{0}\right)$, furnishes a metric of the counts in the Gaussian peak via 


$$
R\left(t_{0}\right)=C_{t o t} \tilde{F}(\sigma, M)-\frac{1}{2} b_{2} M^{3}
$$

where

$$
\begin{aligned}
\tilde{F}(\sigma, M) & \equiv F\left(\tau=t_{0} ; t_{0}, \sigma, M\right) \\
& =2 \operatorname{erf}(Y)-\operatorname{erf}(2 Y) \\
Y & \equiv \frac{M}{2 \sqrt{2} \sigma}
\end{aligned}
$$

and

$$
\operatorname{erf}(x) \equiv \frac{2}{\sqrt{\pi}} \int_{0}^{x} e^{-t^{2}} d t .
$$

In the absence of a quadratic background term (i.e., $\mathrm{b}_{2}=0$ ), the amplitude of the convolute peak is simply proportional to the area of the Gaussian peak, with constant of proportionality $\tilde{F}$. This factor approaches unity asymptotically as the filter width $M \rightarrow \infty$ at fixed Gaussian width, $\sigma$. The effect of a quadratic background component (i.e., non-zero curvature in the background as a function of channel or energy) according to Eq. (11) is to reduce the amplitude of the convolute peak relative to the linearbackground case.

\subsubsection{Peak Area Extraction: Initial Testing of SWC Algorithm}

In preparation for application of the SWC algorithm to actual spectra recorded from $\mathrm{UF}_{6}$ cylinders, we performed initial computational evaluations of several candidate peak-area algorithms as applied to simple synthetic spectra. The primary goals of these early tests were (1) to verify reasonable behavior of the discrete form of the square-wave filter in an easily-interpretable spectroscopic analysis context, and (2) to provide an initial assessment of accuracy and precision of the SWC method in comparison to other candidate peak-area extraction techniques.

The method we adopted for initial testing consisted of synthesizing a set of $N$ (with $N>>1$ ) Poissonblurred, simple single-peak spectra and applying the peak-area algorithms to each spectrum in the set. We recorded the mean and standard deviations of the peak areas thus determined over the pseudospectrum population for each candidate peak-area method. The pseudo-spectra consisted of a single Gaussian peak superimposed on a smooth, quadratic polynomial background. We determined the background parameters by fitting the high-energy edge immediately above the 186-keV region of a typical Areva "background” measurement, recorded from a gravel-filled weight standard cylinder. The ratios of the linear and quadratic coefficients to the constant term in the background polynomial were $b_{1} / b_{0}=-0.012$ and $b_{2} / b_{0}=3.9 \times 10^{-5}$, respectively. This crude background shape was intended to roughly represent relevant background intensities and curvatures in the vicinity of this important energy region without necessarily reproducing in detail the background curvature directly underneath the $186-\mathrm{keV}$ peak.

Evaluation of peak-area algorithm accuracy and precision will, in general, depend upon the model parameters that are allowed to vary freely in describing a sample spectrum. In these initial investigations, we assumed that the position and standard deviation of the Gaussian peak were known, while the amplitude of the Gaussian (and, if relevant to the algorithm, the polynomial background parameters) was 
determined in the spectrum analysis. These are probably the least demanding analysis criteria in terms of algorithm performance and robustness that are still capable of yielding a meaningful test. We evaluated three peak-area algorithms:

1. Compute the convolute of the pseudo-spectrum using the discrete-filter SWC method, then determine the best-fit amplitude of the Gaussian peak required to match the trial convolute shape by varying the amplitude, processing the resulting (digitized) Gaussian shape with the squarewave filter, and matching this "theoretical” convolute shape against the trial spectrum's convolute (in a bin-wise $\chi^{2}$ sense). With the optimum Gaussian amplitude thus determined, and given the known Gaussian $\sigma$, we can compute the area of the peak.

2. Fit the pseudo-spectrum with a Gaussian peak + polynomial background fitting function, then determine the Gaussian peak area from the best-fit amplitude and known $\sigma$.

3. Draw a simple "baseline" under the peak from the background channels at the upper- and lowerenergy extremes of the peak (chosen to lie at $4 \sigma$ from the peak position), then subtract a linear background function from the foreground (background + peak) spectrum. Summing the resulting net spectrum over the region-of-interest defined by the endpoint background subtraction channels then directly yields the peak area.

In these preliminary tests, algorithms (1) and (2) performed very similarly for sets of $N=10^{5}$ pseudospectra at Gaussian peak areas on the order of 30,000 to 40,000 counts (compared to integrated backgrounds of about 120,000 counts, i.e., signal/background 1:4). No statistically-significant bias in the reconstructed peak area was discernible for either (1) or (2), and the standard deviation in the reconstructed area was larger than that expected on the basis of Poisson counting statistics for the (isolated) Gaussian peak by a factor of approximately 2 to 3. (This is due at least in part to variance in the apparent peak counts contributed by the Poisson blurring of the background.) The simple baseline technique was found to be the most susceptible to peak-area bias when the peak rides on a "concaveupward” quadratic background, as the linear baseline assumed in the background subtraction tends to over-predict the background underlying the peak. This effect was on the order of $5 \%$ for the spectrum analysis case examined. The standard deviation in the reconstructed area was also roughly twice as large for the baseline-subtraction method relative to methods (1) and (2), presumably because only two channels (one background channel each at the low- and high-energy extremes of the peak) were used to determine the baseline.

A more rigorous and demanding set of algorithm tests would relax the assumptions of known peak position and energy width and consider a wider range of injected peak areas and shapes, background curvatures, signal/background ratios, and SWC filter widths. A comprehensive head-to-head comparison of algorithm performance must also involve application to sets of realistic spectra recorded from actual $\mathrm{UF}_{6}$ cylinders (or, at least, pseudo-spectra populations synthesized from either empirical or modelingbased templates that reflect reality with reasonable fidelity). However, we deemed the results of these initial investigations to be sufficiently promising to warrant further investigation of the SWC method as the peak-area extraction ingredient in the traditional enrichment-meter algorithm. 


\subsubsection{Application of the SWC to the Enrichment Meter Algorithm}

We applied the discrete form of the square-wave filter to the traditional enrichment meter analysis of the 186-keV peak region in $\mathrm{UF}_{6}$ cylinder data recorded at AREVA in April and May of 2009. In broad outline, analysis of the spectral data for a given spectrometer consists of the following steps:

1. Energy calibration: For the $\mathrm{LaBr}$ and $7.6 \times 7.6 \mathrm{~cm} \mathrm{NaI}$ spectrometers, the channel positions of the 186-keV, 766-keV, and 1001-keV peaks are determined by a "conventional” spectrum analysis approach consisting of a fit to the local peak region with a Gaussian peak + polynomial background function. (We note here for completeness that, for the large $5 \times 10 \times 20 \mathrm{~cm} \mathrm{NaI}$ spectrometer, the energy calibration was extended to span the 3-MeV to 8-MeV high-energy signature region by including the 7.6-MeV peak region (see Figure 6) and, when visible, the 2614-keV peak.) In this sense, the spectrum analysis procedure applied to the AREVA data is not completely decoupled from conventional fitting techniques, although the energy calibration approach described here could be adapted readily to consume peak positions derived from the convolute spectrum, rather than from fits to the raw spectrum. For the NaI spectrometer, a linear fit to the peak positions as a function of energy then suffices to provide a reliable energy calibration ("reliable" in that the 186-keV peak in the convolute spectrum can be correctly identified in all product-, natural-, and depleted-uranium cylinders measured in the 2010 AREVA campaigns). Significant nonlinearity observed in the LaBr spectrometer's peak-position set required use of a quadratic "fit" to the three peaks to preserve reasonable fidelity in the highenergy end of the calibrated energy spectrum (e.g., for purposes of comparing the MCNP model to data; see Section 7.0). However, the calibrated energy of the 186-keV peak was found to be only weakly dependent on the fit order for the $\mathrm{LaBr}$ spectrometer, so that a linear fit would have sufficed for the purpose of implementing the enrichment meter algorithm.

2. Calculation of the SWC for each spectrum, identification of convolute peaks and determination of the convolute peak amplitude for the $186-\mathrm{keV}$ peak: We calculate the SWC spectrum from the raw spectrum using the formulae outlined in Section 2.1.1 above. Peaks in the convolute spectrum are identified using a simple crest-above-threshold algorithm that determines the positions (expressed in channel units) of local maxima above a user-specified threshold. Of primary interest in this peak-selection algorithm is the survival of the 186-keV peak as a "candidate" for the next stage of peak identification. In practice, we found that it was possible to specify a single convolute threshold (specific to the spectrometer, i.e., one for $\mathrm{LaBr}$ and one for the $7.6 \times 7.6 \mathrm{~cm}$ $\mathrm{NaI}$ ) that assured selection of the 186-keV convolute peak over the full range of measured \{product, natural, depleted stock\} cylinders recorded in the 2010 AREVA campaigns. With a set of candidate peaks in hand, and the energy calibration as determined in step (1) above, the identification algorithm then determines the peak that most closely matches the energy of the 186-keV peak of interest. The software implementing our algorithm aborts the analysis if, in fact, no peak matching the target 186-keV energy (to within a small user-specified tolerance) can be found. The amplitude of the $186-\mathrm{keV}$ convolute peak is then determined simply by recording the convolute value in the peak channel. We can envision more sophisticated convolute peak analysis techniques, e.g., a fit to the full convolute peak region exploiting the known analytical shape of the convolute for a Gaussian input-spectrum peak. For these feasibility investigations, however, we adopted essentially the simplest possible approach to meaningfully implement the convolute-based approach. 
3. Determination of the enrichment-meter calibration curve: For a given spectrometer, we then determine the enrichment calibration curve by fitting a line to the set of $186-\mathrm{keV}$ convolute peak amplitude values (with associated statistical uncertainties derived from Poisson counting statistics in the raw input spectrum) as a function of the declared enrichment. We can then easily determine the enrichment of a sample cylinder relative to this calibration curve from an observed convolute peak amplitude simply by inverting the linear fit. We note in passing that strict linearity of the calibration curve is not a prerequisite for implementing the enrichment-meter technique (or, indeed, an arbitrary assay method that relies on the correlation of some physical observable with the isotopic content of the sample). To date, only linear calibration curves have been applied to the analysis of the convolute-based approach to the enrichment-meter method. However, the possibility of accounting for small departures from linearity with a quadratic, or higher-order, calibration curve may warrant future investigation as the method matures. These departures may be especially evident at low enrichment, where curvature in the background distribution as a function of energy may noticeably impact the "first-order" convolute amplitude metric described in step (2) above. See further discussion of possible strategies to mitigate background curvature effects in Section 9.0 below.

4. Calculation of the relative standard deviation of the assay results with respect to the declared enrichments: The primary statistical metric adopted in this project to summarize dispersion of the enrichment calibration results is the standard deviation of the relative, or fractional, difference of assayed enrichments from declared. In general, this standard deviation may be computed for a different subset of the overall population of cylinder measurements than was used to determine the calibration curve. For example, one may analyze the set of all relevant \{product, natural, depleted cylinder measurements in a given field campaign to determine the calibration curve, and then separate relative standard deviations computed for the product, natural, and depleted subpopulations. We adopted this approach for results quoted in Section 5.0.

\subsection{Non-Traditional High-Energy Gamma Rays as Indirect Measure of ${ }^{235} \mathrm{U}$}

Walton [1974] and Reilly [1974] previously studied the potential use of total neutron count rate as a means of determining $\mathrm{UF}_{6}$ enrichment but this signature is not currently used in IAEA verification measurements. Neutron-based signatures are attractive because of the high penetrability of neutrons from the interior of the cylinder volume to the outside wall. The fraction of neutrons escaping the cylinder wall ranged from 0.80 to 0.90 in previous studies [Walton 1974], with weak dependence on the cylinder enrichment and fill level. Berndt [2010]also discusses the impact of fill volume and the configuration of the $\mathrm{UF}_{6}$ within the cylinder on neutron signatures .

The primary source of neutrons from product cylinders is ${ }^{19} \mathrm{~F}(\alpha, n)$ reactions, with the dominant alpha emitter being ${ }^{234} \mathrm{U}$ for all enrichments above natural. Sources of neutrons other than ${ }^{234} \mathrm{U}$ include ${ }^{238} \mathrm{U}$ spontaneous fission and alpha emission, and induced fission in ${ }^{235} \mathrm{U}$. Figure 5 plots the relative contributions of neutron source terms for 30B cylinder enrichments ranging from depleted $\left(0.2 \mathrm{wt} \%{ }^{235} \mathrm{U}\right)$ to the Type 30B limit of $5 \mathrm{wt} \%{ }^{235} \mathrm{U}$. In these calculations, we used SOURCES 4C [Wilson 2002] to determine the yield (and spectral shape) of the $(\alpha, n)$ and spontaneous fission neutron production from ${ }^{234} \mathrm{U}$ and ${ }^{238} \mathrm{U}$. The $(\alpha, n)$ yields from $U_{6}$ were 602 neutrons $/ \mathrm{s} / \mathrm{g}{ }^{234} \mathrm{U}$ and 0.0119 neutrons $/ \mathrm{s} / \mathrm{g}{ }^{238} \mathrm{U}$. We calculated the ${ }^{238} \mathrm{U}$ spontaneous fission to be $0.0136 \mathrm{n} / \mathrm{s} / \mathrm{g}{ }^{238} \mathrm{U}$. We ignored the ${ }^{235} \mathrm{U}$ contributions (i.e., $(\alpha, n)$, spontaneous fission and induced fission) in the Figure 5 calculations because they are negligible in 
comparison to ${ }^{234} \mathrm{U}$ and ${ }^{238} \mathrm{U}$ contributions, over this enrichment range [Reilly 1991]. We took the density of the $\mathrm{UF}_{6}$ to be $5.1 \mathrm{~g} / \mathrm{cm}^{3}$ and assumed a 30B cylinder to be filled to $60 \%$ of its total volume, consistent with mass limits for that cylinder type [American National Standards Institute 1995]. The ${ }^{234} \mathrm{U} /{ }^{235} \mathrm{U}$ ratio varied with enrichment according to calculations performed by Fischer for a modern gaseous centrifuge enrichment plant (GCEP) [Fischer 2005] and consistent with environmental samples taken from an operational GCEP [Cooley 2000].

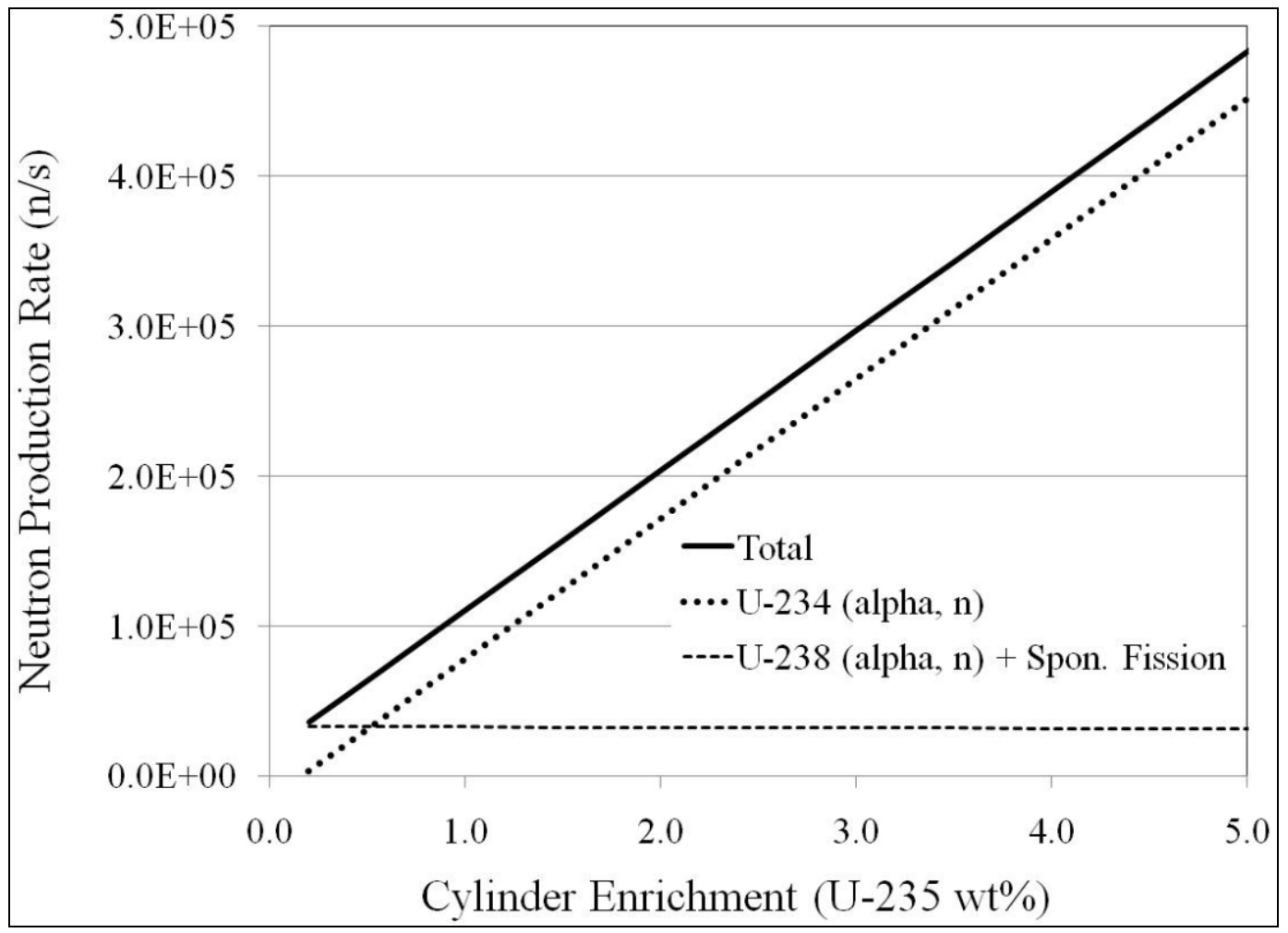

Figure 5. Calculated neutron production rate in a Type 30B cylinder. The ${ }^{234} \mathrm{U}$ is the dominant source term for alpha particles and subsequent neutrons (i.e. ${ }^{19} \mathrm{~F}(\alpha, n)$ ) above approximately $0.5 \mathrm{wt} \%$ ${ }^{235} \mathrm{U}$. The ${ }^{238} \mathrm{U}$ (alpha emissions combined with the ${ }^{19} \mathrm{~F}(\alpha, n)$ reaction) and spontaneous fission source terms are approximately equal over all enrichments and are summed in this figure. We ignored ${ }^{235} \mathrm{U}$ contributions here since they are comparatively insignificant.

Figure 5 shows that ${ }^{234} \mathrm{U}$ alpha emission (combined with the ${ }^{19} \mathrm{~F}(\alpha, \mathrm{n})$ reaction) is the predominant source of neutrons above enrichments of approximately $0.5 \%$ and that total neutron production rate varies linearly between approximately $3 \times 10^{4}$ and $5 \times 10^{5} \mathrm{n} / \mathrm{s}$ over an enrichment of $0.2 \%$ to $5 \%$. The neutron production rate from ${ }^{238} \mathrm{U}$ is relatively stable for cylinders in the enrichment range studied here (up to $5 \%$ ${ }^{235} \mathrm{U}$ ), as the total mass of ${ }^{238} \mathrm{U}$ changes by less than $5 \%$ over that range. These results for relative contributions and total production rate are consistent with previous calculations and measurement results [Reilly 1991; Walton 1974; Reilly 1974]. Previous studies have indicated that the linearity of the neutron production rate will not be preserved for enrichments greater than $20 \%$ due to neutron multiplication with ${ }^{235} \mathrm{U}$ fission.

The neutrons produced in the $\mathrm{UF}_{6}$ interact in the $\mathrm{UF}_{6}$ itself, surrounding steel cylinder and adjacent structural materials, as well as in materials in close proximity to gamma-ray spectrometers. Those 
interactions (e.g., inelastic scatter and neutron capture) can induce high-energy gamma-ray signatures that extend to energies greater than $10 \mathrm{MeV}$. Prominent among these, due to the large volume of steel in the cylinder assay scenario, are the 7.631-MeV (100\%) and 7.645-MeV (86\%) lines from neutron capture reactions on ${ }^{56} \mathrm{Fe}$ (91.8\% natural abundance). The steel wall of the cylinder and steel (or other materials) located near the gamma-ray spectrometers, therefore, serve as neutron-to-gamma-ray converters.

This high-energy gamma-ray signature is useful for several reasons. First, it allows exploitation of a neutron signature without dedicated neutron sensors, thereby simplifying system design and reducing cost-particularly advantageous with the current shortage of ${ }^{3} \mathrm{He}$. Second, the goal of full-volume assay is achieved by virtue of the penetrability of the neutrons (escape fraction from the $\mathrm{UF}_{6}$ volume of greater than $80 \%$ ) that induce the gamma rays. Finally, the high-energy gamma-ray signature is well above the energy range of the more familiar emissions from $\mathrm{UF}_{6}$ (e.g., $1001 \mathrm{keV}$ from the ${ }^{234 \mathrm{~m}}$ Pa daughter of ${ }^{238} \mathrm{U}$ and $2614 \mathrm{keV}$ from ${ }^{208} \mathrm{Tl}$, daughter of ${ }^{232} \mathrm{U}$ ) and essentially all natural background source terms (e.g., $2614 \mathrm{keV}$ from ${ }^{208} \mathrm{Tl}$, daughter of ${ }^{232} \mathrm{Th}$ ). Though these high-energy gamma rays are considerably more penetrating than the $185-\mathrm{keV}$ and $1001-\mathrm{keV}$ often associated with $\mathrm{UF}_{6}$ assay, they are still unable to penetrate through any significant distance of $\mathrm{UF}_{6}$ relatively to the $76 \mathrm{~cm}$ diameter of the 30B cylinder: the mean free path of an 8-MeV photon in $U_{6}$ at $5.1 \mathrm{~g} / \mathrm{cm}^{3}$ is approximately $4 \mathrm{~cm}$. Consequently, a gammaray spectrometer on one side of the cylinder sees little or no contribution from neutron-induced gamma rays produced by the wall on the far side of the cylinder.

Because the full-energy efficiency for $8 \mathrm{MeV}$ gamma rays in practicable scintillator crystal sizes (i.e., $100 \mathrm{~cm}^{3}$ or less) is quite low, the majority of recorded counts are from Compton scatter or pair production, followed by escape of one or more of the annihilation photons. Therefore, we used an energy window of approximately 3.0 to $8.0 \mathrm{MeV}$ in this work to tally gamma-ray counts attributed to neutron emission from the cylinder. While measurements and modeling indicate that this region of interest is dominated by the emission doublet at 7.631 and $7.645 \mathrm{MeV}$ from capture in ${ }^{56} \mathrm{Fe}$, other lower-intensity gamma rays produced by neutron interactions in iron and other structural materials may also contribute.

The overall intensity of the high-energy gamma-ray signature, and therefore the assay time required to achieve the desired uncertainties, is dependent on the volume of steel (or other converter materials) within the spectrometer's field of view, which could include spectrometer collimator designs engineered to facilitate the neutron-to-gamma-ray conversion. That volume of steel also contributes to the variability in the intensity of the signature. If the volume, elemental composition and geometric configuration of steel in the system (i.e., cylinder, trolley, spectrometer collimators) is consistent across cylinder measurements, it is possible to preserve the desired proportionality between neutron flux and neutroninduced gamma-ray flux.

Utilizing this neutron-derived signature as an indirect measure of ${ }^{235} \mathrm{U}$ requires that the ${ }^{234} \mathrm{U} /{ }^{235} \mathrm{U}$ ratio be known. Work by Richter et al. indicates that this is generally true for natural-uranium feed material ( $\sim \%$ variation in the ratio), based on mass spectrometry measurements of various uranium ore samples from around the world [Richter 1999]. Other studies have indicated that the isotopic ratio may vary significantly with enrichment process, particularly for enrichments above $5 \mathrm{wt} \%{ }^{235} \mathrm{U}$ [Reilly 1991; Reilly 1974]. However, the variation of the ratio within a multiple-cascade GCEP is relatively low, according to the analysis of environmental samples described in [Cooley 2000]. These studies provide some support for the assertion that under assumptions of ore-based feed and a facility-specific calibration, the nontraditional signature can be a reliable measure of cylinder enrichment. 
Some enrichment-industry practices, however, may violate these assumptions. For example, modern enrichment plants, depending on the price of uranium, may also recycle tails as feed material, potentially altering the ${ }^{234} \mathrm{U} /{ }^{235} \mathrm{U}$ ratio in the product cylinders. Yet another potential source of variation in this ratio is the use of reactor-recycle uranium. Section 8.2 discusses the potential impacts of ${ }^{234} \mathrm{U} /{ }^{235} \mathrm{U}$ ratio variation on the hybrid (i.e., traditional + non-traditional) NDA methods in more detail.

Figure 6 illustrates a comparison of how gamma-ray signatures present themselves in different types of gamma-ray spectrometers. The small handheld HPGe, typical of today's IAEA instruments, has a lower peak-to-total ratio at energies above a few hundred keV, and has virtually no collection efficiency above $2 \mathrm{MeV}$. The 120\% HPGe has much improved high-energy efficiency and reveals the ${ }^{56} \mathrm{Fe}$ neutron capture lines in the 7.6-MeV region. The two $\mathrm{NaI}(\mathrm{Tl})$ sensors have much higher overall collection efficiency, higher peak efficiency above a few hundred kiloelectron volts, and have sufficient stopping power to detect signatures ranging to $8 \mathrm{MeV}$.

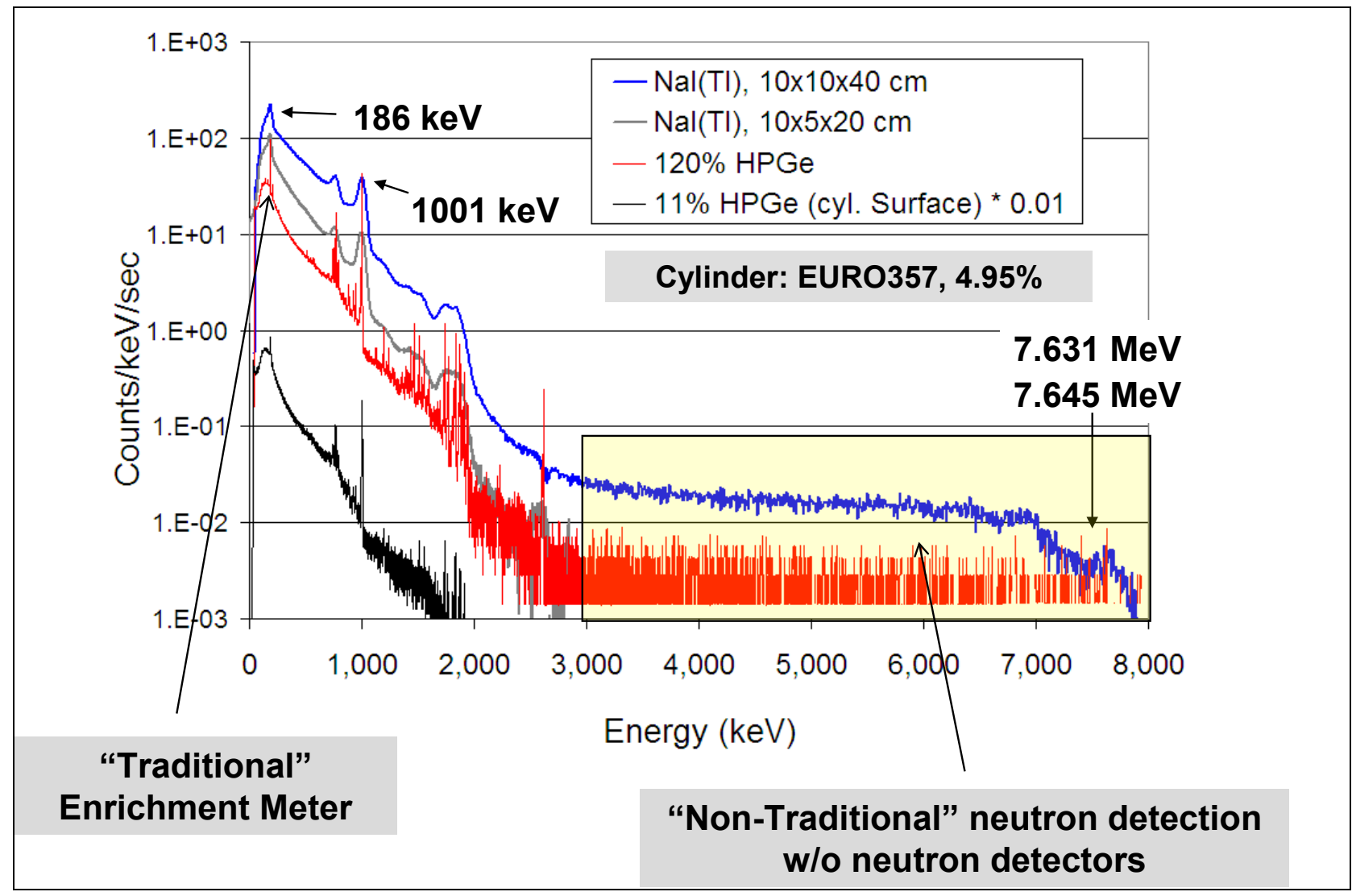

Figure 6. Spectra recorded by two large NaI(Tl) spectrometers and two HPGe spectrometers from a 30B cylinder enriched to 4.95\%. The higher interaction efficiency of the $\mathrm{NaI}(\mathrm{Tl})$ enables the collection of neutron-induced high-energy signatures. In this work, that signature is defined with a window between 3.0 and 8.0 MeV (yellow box). 
PNNL-19854

\subsection{Field Prototype}

An instrumentation prototype was developed for cylinder measurements that could be used to support an evaluation of the hybrid NDA methods described above. The prototype (see Figure 9) includes two small-volume and one large-volume spectrometer.

The smaller spectrometers are a $3.8 \times 3.8 \mathrm{~cm} \mathrm{LaBr}(\mathrm{Ce})$ (hereafter, "LaBr") and a $7.6 \times 7.6 \mathrm{~cm}$ $\mathrm{NaI}(\mathrm{Tl})$ (hereafter, "NaI”). The energy resolutions of the $\mathrm{LaBr}$ and $7.6 \times 7.6 \mathrm{~cm} \mathrm{NaI}$ were $2.4 \%$ and $6.5 \%$ at $662 \mathrm{keV}$, respectively. Ideally, these two spectrometers would simultaneously record both the low- and high-energy signatures. Achieving this goal, however, creates difficult pulse-processing requirements for medium-resolution scintillators. For example, the $\mathrm{LaBr}$ spectrometer has a full-width-at-half-maximum (FWHM) of approximately $9 \mathrm{keV}$ at $186 \mathrm{keV}$. Faithfully capturing this peak shape for spectrometric analysis requires at least four to five channels to span that FWHM [Knoll 2000]. This translates to channel widths of $2 \mathrm{keV}$ or less. If an upper energy limit of approximately $9 \mathrm{MeV}$ is also needed, a minimum 12-bit (4096 channels) analog-to-digital converter is required. While 12-bit (and higher) ADCs are available in integrated pulse-processing systems (i.e., preamplifier, digital shaping and high-voltage) for HPGe spectrometers, that is not the case for scintillator pulse-processing systems (HPGe-focused systems generally cannot provide sufficient current in the high-voltage supply for photomultiplier tubes). Time and budget constraints precluded the purchase of pulse-processing electronics that could achieve the ideal. Instead, we utilized Ortec DigiBases (1024 channels) and configured the $\mathrm{LaBr}$ and $7.6 \times 7.6 \mathrm{~cm}$ $\mathrm{NaI}$ to record a maximum energy of approximately $1300 \mathrm{keV}$. In this way, we used them to analyze only the low-energy traditional signatures.

We introduced a third detector to record the high-energy signature, again using a DigiBase bit now with a channel width of approximately $9 \mathrm{keV}$ to achieve a maximum deposited energy of about $9 \mathrm{MeV}$. This spectrometer is a $5.1 \times 10.2 \times 20.3 \mathrm{~cm} \mathrm{NaI}$ (middle detector) wrapped in an iron/polyethylene collimator designed to enhance the conversion of neutrons to high-energy gamma rays. The iron layers are each $0.64 \mathrm{~cm}$ thick, while the polyethylene layers are $1.28 \mathrm{~cm}$ thick. In frontal area, this larger detector approximates two $7.6 \times 7.6 \mathrm{~cm} \mathrm{NaI}$ spectrometers; in volume, three of those sensors, making it a reasonable surrogate for the high-energy gamma-ray signature that would be recorded by an array consisting of three $7.6 \times 7.6 \mathrm{~cm} \mathrm{NaI}$ spectrometers, a notional design for the ICVS. The energy resolution of the larger $\mathrm{NaI}$ spectrometer is $8.0 \%$ at $662 \mathrm{keV}$.

\subsection{Calibration and Characterization of Gamma-Ray Spectrometers}

Prior to the field measurements, we performed laboratory calibration and characterization measurements on the spectrometers. These measurements included a study of resolution as a function of shaping time (Ortec DigiBases, bipolar shaping) and high voltage applied to the photomultiplier tubes, with the goal being to identify suitable instrument operating windows (Figure 7, top) [Knoll 2000]. Once we identified suitable settings, we used a series of check sources to assess the expected energy resolution (relative FWHM) as a function of energy (Figure 7, bottom), in a manner consistent with similar work by others [Moszynski 2002; Ciemala 2009; Nicolini 2007; Quarati 2007]. 

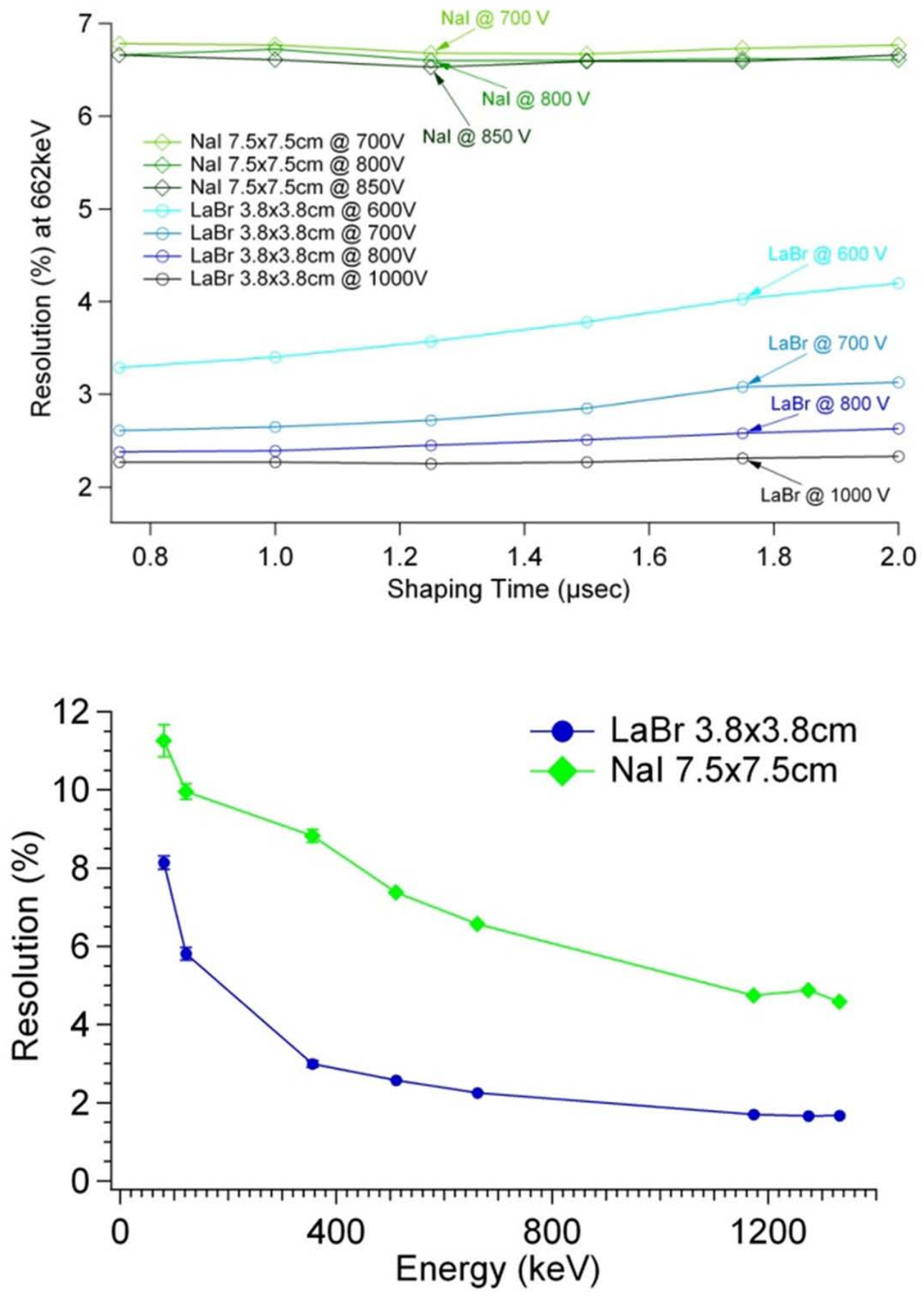

Figure 7. Energy resolution for the $\mathrm{LaBr}$ and $\mathrm{NaI}(7.6 \times 7.6 \mathrm{~cm})$ spectrometers as a function of bipolar shaping time and high-voltage (top) and incident energy (bottom) at appropriate shaping and high-voltage settings

Due to relatively high count rates expected in the cylinder assay scenario, we made further measurements to evaluate the resolution of each detector as a function of incident count rate. We plotted 
the recorded count rate against the estimated incident count rate on the face of each detector (see Figure 8). These measurements supported design decisions for the field prototype (e.g., collimation choices to limit dead time). Figure 8 (top) shows how the recorded count rate in each detector varies as the incident count rate increases and Figure 8 (bottom) shows how the resolution of each detector is affected by the increasing rate. The resolution plots are based on a Gaussian fit of the ${ }^{137} \mathrm{Cs}$ peak at $662-\mathrm{keV}$ and the error bars are based on a one-sigma variation in the fit parameter FWHM. The relatively large error bars and scatter in the resolution data at count rates below approximately $5 \times 10^{3}$ cps are due to the fairly poor counting statistics of those experiments (weak source located approximately $100 \mathrm{~cm}$ from the spectrometers). 


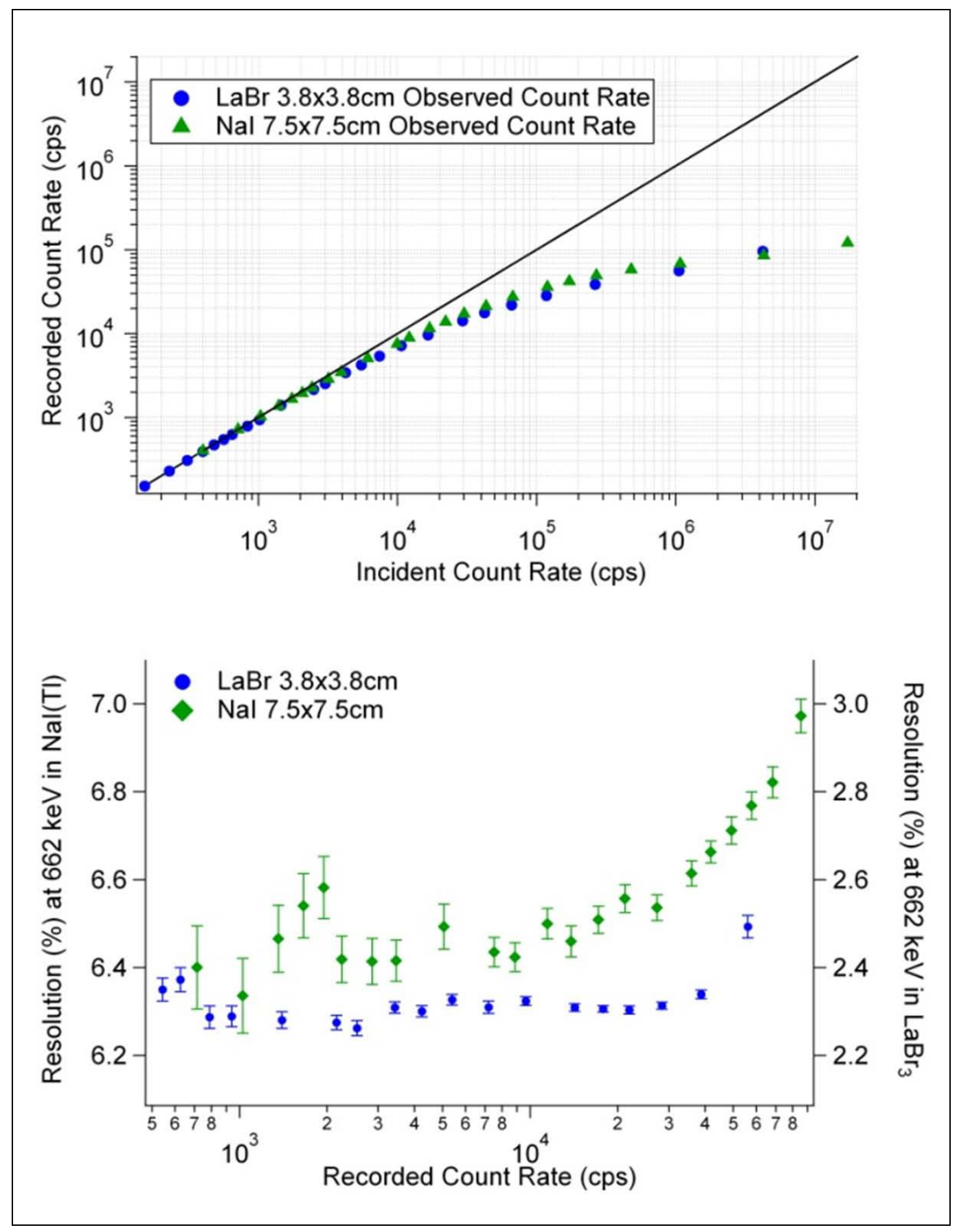

Figure 8. Top: Observed count rate as a function of the incident count rate. Bottom: Energy resolution at $662-\mathrm{keV}$ as a function of incident count rate.

The data in Figure 8 follow the trends expected for these particular medium-resolution scintillators. The $\mathrm{NaI}$ and $\mathrm{LaBr}$ recorded count rates are comparable since both used bipolar shaping with comparable time constants ( $1.00 \mu$ s for $\mathrm{LaBr}$; $1.25 \mu$ s for $\mathrm{NaI})$. However, because of the longer scintillation light decay time in NaI, we observe that the increasing count rate on energy resolution degrades at lower rates in this crystal than for the $\mathrm{LaBr}$, which has a shorter scintillation decay time [Smith 2010]. At the rates of 
interest to this project, generally less than $1 \times 10^{5} \mathrm{cps}$, both spectrometers exhibit predictable recorded count rate behavior and relatively stable energy resolution (less than $10 \%$ relative change).

\subsection{Collimation and Neutron-to-Gamma Converters}

We fabricated and utilized two different collimator types in order to address two research questions:

1. What traditional-assay performance can be realized with a collimator design focused on collecting only the low-energy signatures? For these measurements and analysis, we surrounded the two spectrometers with cylindrical lead collimators with a wall thickness of $2.2 \mathrm{~cm}$ and aperture diameters of $5.1 \mathrm{~cm}$ and $7.6 \mathrm{~cm}$ for the $\mathrm{LaBr}$ and $(7.6 \times 7.6 \mathrm{~cm})$ NaI, respectively.

2. What traditional-assay performance can be realized with a collimator design focused on collecting and enhancing the non-traditional high-energy gamma-ray signatures? For these measurements and analysis, we surrounded the two spectrometers with cylindrical collimators consisting of $0.64 \mathrm{~cm}$ of steel and $1.28 \mathrm{~cm}$ of polyethylene. This collimator design is intended to enhance the high-energy gamma-ray signature by moderating neutrons in the vicinity of the detector and providing an iron conversion layer. This collimator also included a lead aperture on the front face (0.64-cm thick) and 0.32-cm lead wrapped around the outside of the polyethylene. This lead structure provides substantial collimation of the $186-\mathrm{keV}$ (less than $0.1 \%$ uncollided penetration through $0.64 \mathrm{~cm}$ of lead) while minimizing the attenuation of the $7.6-\mathrm{MeV}$ gamma rays (less than $3 \%$ attenuation at $0.64 \mathrm{~cm}$ ). The lead aperture diameters were $5.1 \mathrm{~cm}$, and $7.6 \mathrm{~cm}$ for the $\mathrm{LaBr}$ and $(7.6 \times 7.6 \mathrm{~cm}) \mathrm{NaI}$, respectively. 


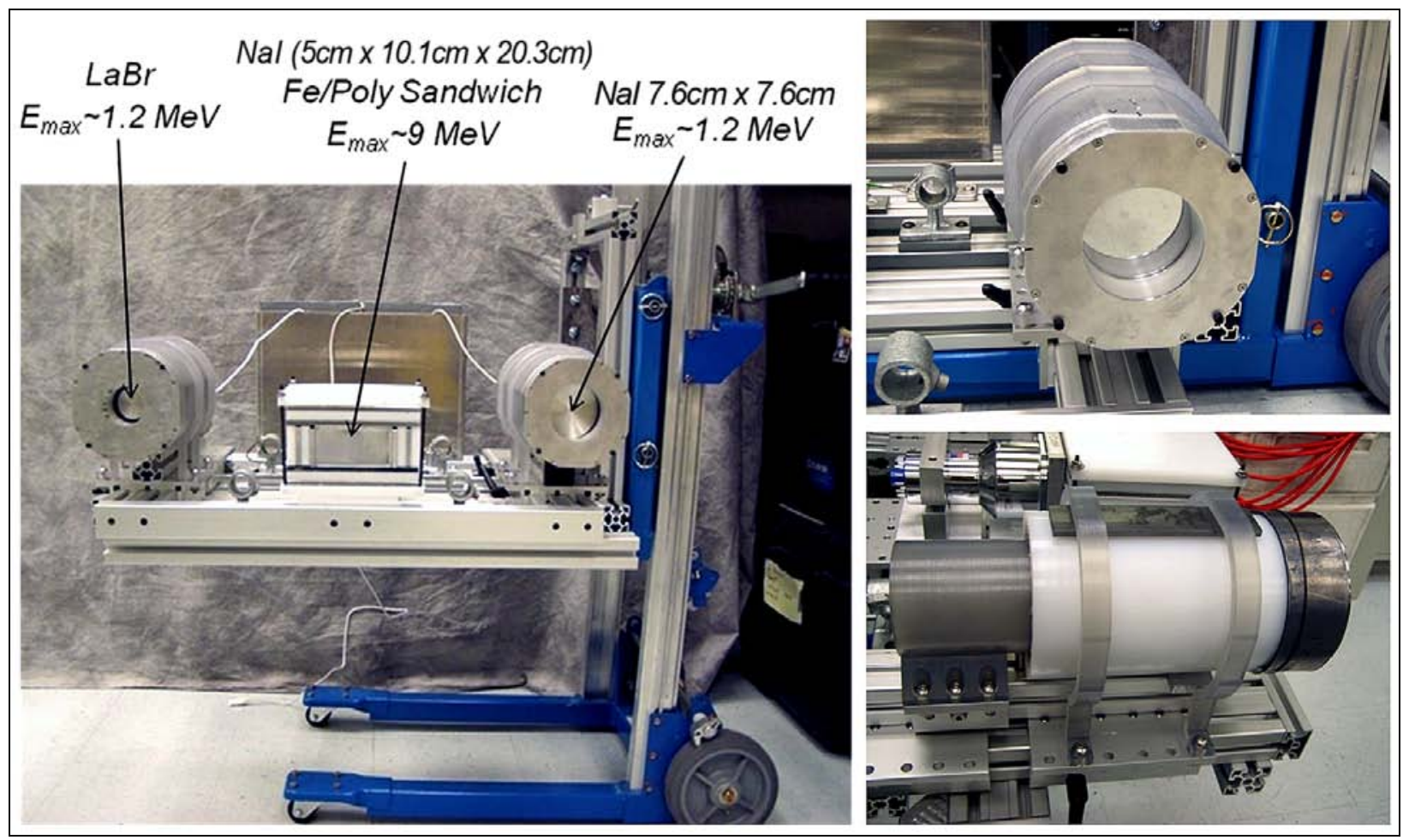

Figure 9. Left: Field demonstration prototype that includes collimated three $8 \times 3.8 \mathrm{~cm} \mathrm{LaBr}$ and $7.6 \times 7.6 \mathrm{~cm} \mathrm{NaI}$ spectrometers, and a $5.1 \times 10.2 \times 20.3 \mathrm{~cm} \mathrm{NaI}$ (middle detector) wrapped in layers of iron and polyethylene. Right: Lead (top) and Fe/poly (bottom) collimator designs used for the $\mathrm{LaBr}$ and $7.6 \times 7.6 \mathrm{~cm} \mathrm{NaI}$ spectrometers. The Fe/poly cylindrical collimator (with lead end cap aperture) is intended to enhance the high-energy gamma-ray signature.

The field prototype system also includes two polyethylene slabs $(2.5 \times 30 \times 122 \mathrm{~cm})$ intended to reflect neutrons emitted by the cylinder back toward the cylinder (see Figure 9), thereby increasing the neutron to high-energy gamma-ray conversion process. MCNP modeling showed that the Fe/Poly collimator increased the high-energy gamma-ray signature (counts summed between 3.0 to $8.5 \mathrm{MeV}$ ) by approximately $19 \%$ over the bare detector case, and that the polyethylene fence provided an additional $16 \%$ increase. Together, the two design features translate to a $38 \%$ increase in the intensity of the nontraditional signature and a commensurate improvement in the statistical precision that can be realized for a given assay time (e.g., 5 minutes). 


\subsection{Field Measurements}

PNNL performed a cylinder measurement campaign at an AREVA NP fuel fabrication plant in Richland, Washington. We used the Fe/poly collimators on all three spectrometers to measure the cylinder population that is the focus of this paper. The 26 cylinders consisted of 23 product ( 2 to 5 wt $\%$ $\left.{ }^{235} \mathrm{U}\right)$, 2 natural enrichment $\left(0.711 \mathrm{wt} \%{ }^{235} \mathrm{U}\right)$, and 1 depleted (approximately $0.2 \mathrm{wt} \%{ }^{235} \mathrm{U}$ ) cylinder. We also measured several highly enriched uranium (HEU)-downblend and partially filled cylinders during the campaign were, but they were not included in the predictions of performance for the ICVS NDA methods on the premise that the potential of the NDA methods first needs to be understood for the cylinder types most likely to be encountered in IAEA verification scenarios (i.e., ore-based feed and cylinders filled to capacity). Figure 10 shows a photograph of the experimental setup at the AREVA plant is shown in and Figure 11 gives example cylinder assay spectra.

We took background measurements (approximately 45 minutes) with a cylinder-weight standard (filled with gravel) on the trolley system so that the recorded background incorporated the shielding that the cylinder being interrogated provides from the nearby field of $\mathrm{UF}_{6}$ cylinders. We subtracted the background count rates (186-keV peak region for the traditional, 3.0- to $8.0-\mathrm{MeV}$ window for the nontraditional) from the measured cylinder data as a part of the analysis described in the next section. 


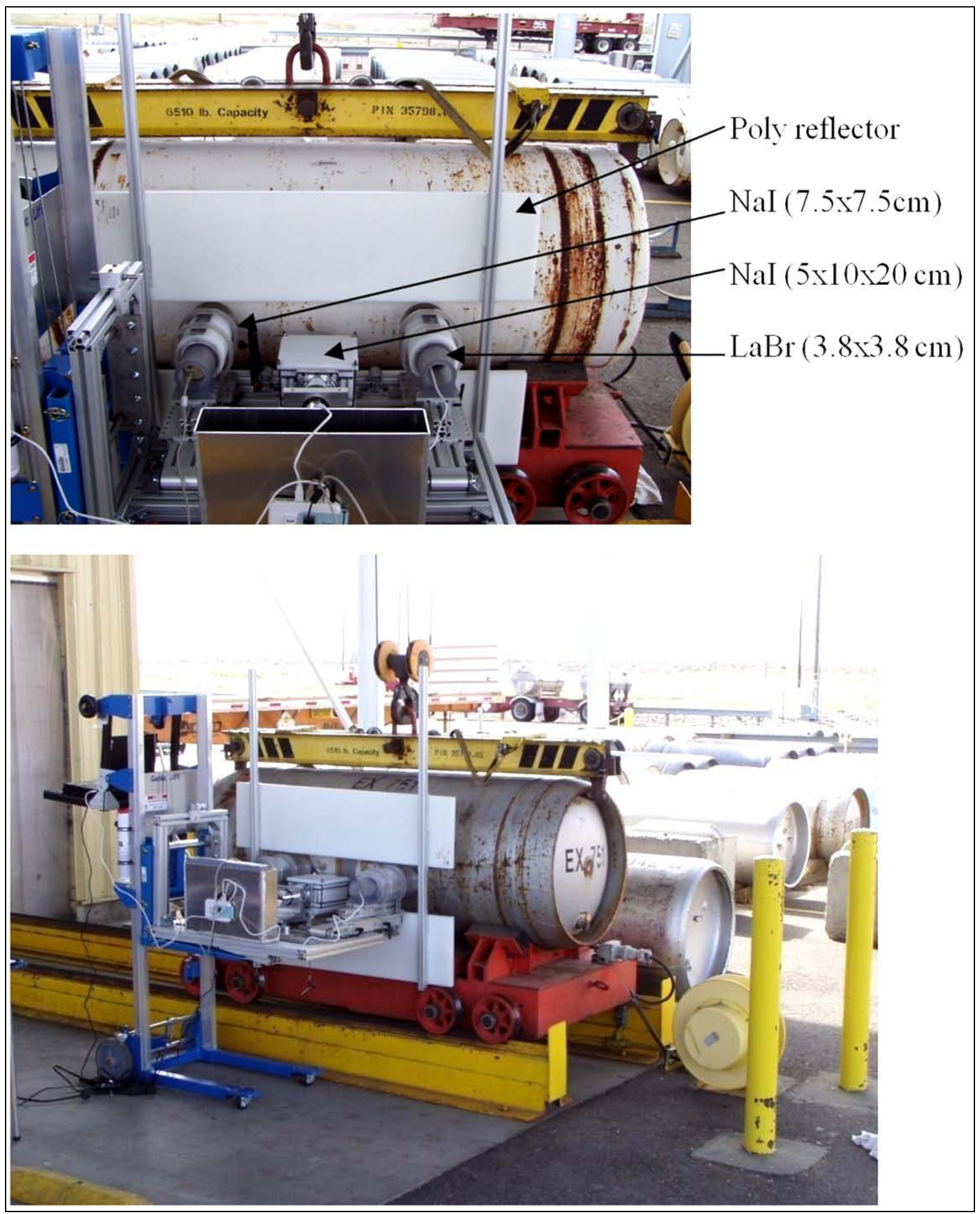

Figure 10. Photographs of experimental setup at AREVA NP fuel fabrication facility 
PNNL-19854

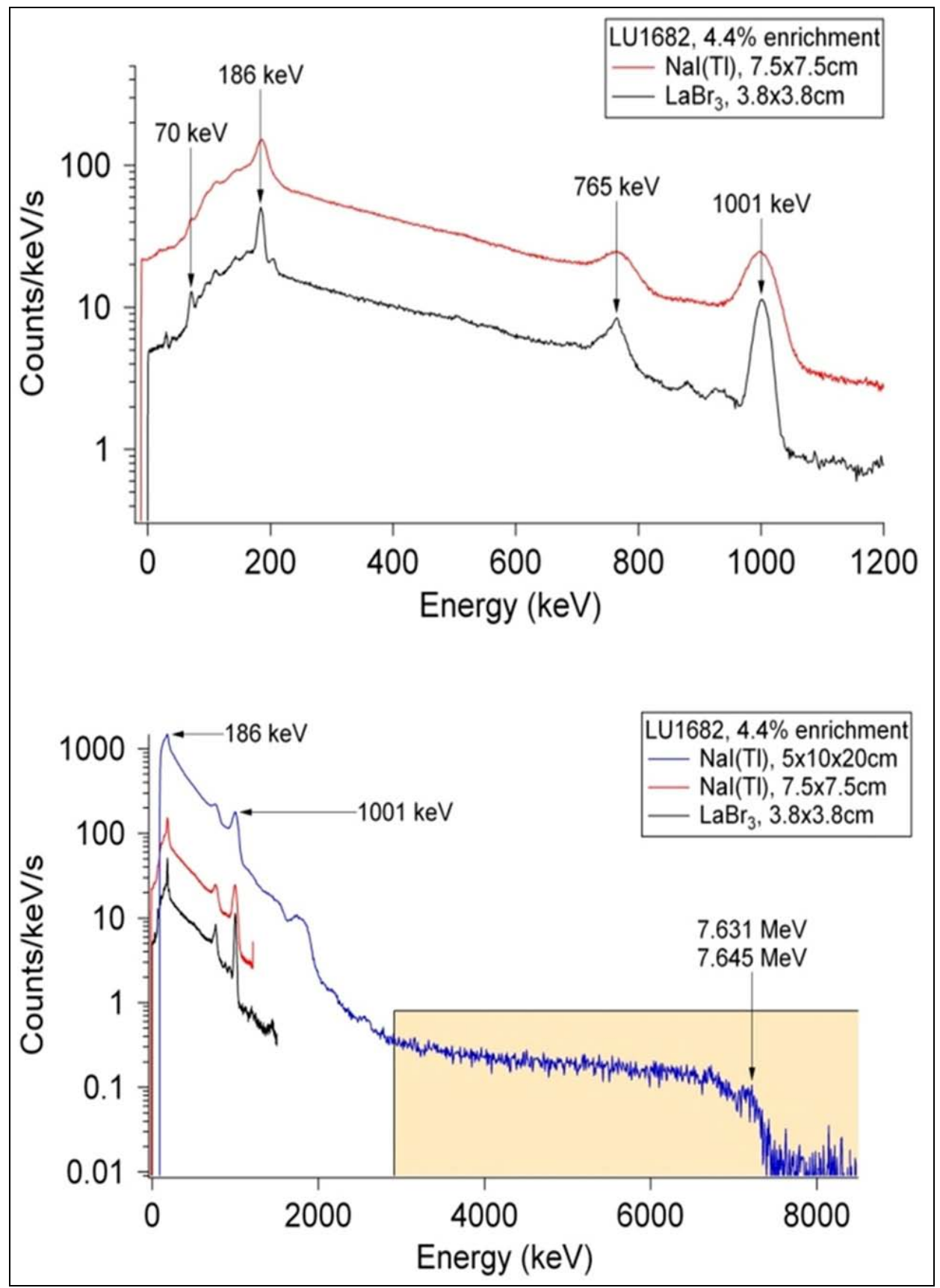

Figure 11. Example spectra recorded from a 30B cylinder enriched to $4.4 \mathrm{wt} \%{ }^{235} \mathrm{U}$. Left: $3.8 \times 3.8 \mathrm{~cm}$ $\mathrm{LaBr}$ and $7.6 \times 7.6 \mathrm{~cm} \mathrm{NaI}$ for energies to $1.2 \mathrm{MeV}$. Right: $5 \times 10 \times 20 \mathrm{~cm} \mathrm{NaI}$ for energies to approximately $8 \mathrm{MeV}$.

The AREVA cylinder population not only spans a wide range of enrichments, but is also diverse in terms of the enrichment facilities that produced the cylinders. Enrichment providers included Urenco, USEC, Eurodif and Ural Electrochemical Integrated Plant. The diversity of enrichment feeds, sources and processes in the AREVA cylinders presented a relatively difficult test case for the non-traditional assay methods that assume a known ${ }^{234} \mathrm{U} /{ }^{235} \mathrm{U}$ ratio and would utilize a facility-specific calibration. 


\subsection{Results: Cylinder Assay}

As discussed previously, initial development of ICVS NDA methods assumes a facility-specific calibration and ore-based feedstock. Consequently, HEU-downblend cylinders are not included in this analysis, nor are partially-filled cylinders. The potential for the assay of cylinders produced from other feedstock (e.g., tails recycle, reactor recycle and HEU-downblend) and partial cylinders is a subject of ongoing investigation and work to date is summarized in Section 8.0.

The following text and figures give enrichment assay results using the 26-cylinder population described previously. In this analysis, the figure of merit for assay accuracy is the relative standard deviation of the assay enrichment values (either in mass or wt $\%{ }^{235} \mathrm{U}$ ) relative to the declared values for each cylinder. Linear calibrations translate the intensity of the assay signature to a corresponding ${ }^{235} \mathrm{U}$ mass (or enrichment) value.

\subsection{Traditional Assay}

Figure 12 shows the assay results for the traditional enrichment meter method implemented using the SWC algorithm for the extraction of net peak count rate for the $\mathrm{LaBr}$ and the $7.6 \times 7.6-\mathrm{cm} \mathrm{NaI}$ spectrometers, with the Fe/poly collimator/converter design. The live times were 400 seconds for the $\mathrm{LaBr}$ and 300 seconds for the NaI. The one-sigma error bars in these plots reflect only the statistical counting uncertainty (not the systematic uncertainty in the fitting algorithm) in the net peak count rate. This statistical uncertainty is significantly less than the overall standard deviation for nearly all of the cylinders, as expected, since systematic uncertainties such as wall-thickness variation and nonlinear continuum subtraction are generally the largest source of uncertainty in the traditional enrichment-meter approach.

The relative standard deviations from the declared ${ }^{235} \mathrm{U}$ enrichments for the 23 product cylinders are 3.1\% for $\mathrm{LaBr}$ and 3.5\% for NaI; for the two natural-enrichment cylinders, 9.4\% for $\mathrm{LaBr}$ and $7.9 \%$ for NaI; for the depleted cylinders, $11 \%$ for $\mathrm{LaBr}$ and $13 \%$ for $\mathrm{NaI}$. The $\mathrm{LaBr}$ and $\mathrm{NaI}$ performance is comparable over the entire enrichment range (0.2 to $\left.5.0 \mathrm{wt} \%{ }^{235} \mathrm{U}\right)$, but the number of natural and depleted cylinders is very limited.

While the Fe/poly collimator design is the more appropriate for a hybrid enrichment assay station also collecting the non-traditional signature, comparison with the results from the lead collimator design used in the April 2010 measurements provides indication of how the traditional enrichment meter performance is compromised by using a collimator designed to enhance the non-traditional signature. For the lead collimators, the relative standard deviations from the declared ${ }^{235} \mathrm{U}$ enrichments for the 16 product cylinders are 3.2\% for $\mathrm{LaBr}$ and 3.8\% for NaI. As with the Fe/poly case, the $\mathrm{LaBr}$ and NaI performance is comparable and the difference between the two collimators' configurations is relatively small. 
PNNL-19854
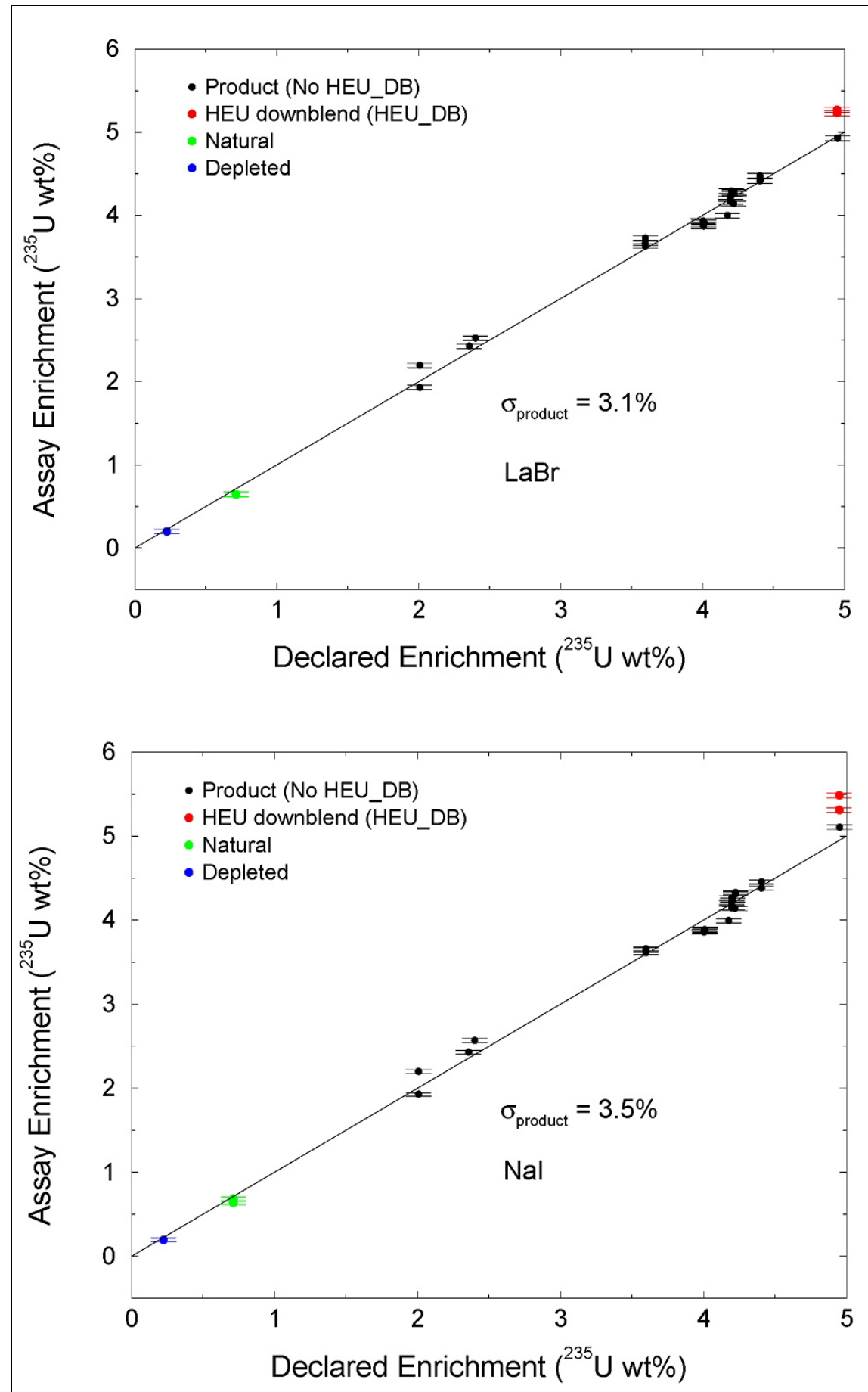

Figure 12. Assay enrichment versus declared enrichment using the traditional enrichment-meter method and a square-wave convolute algorithm for the calculation of net count rate in the 186-keV peak: $\mathrm{LaBr}$ (Top) and $\mathrm{NaI}$ (Bottom). The analysis data set spans 26 full cylinders (0.2 to $5 \mathrm{wt}$ $\%{ }^{235} \mathrm{U}$ ) but does not include the HEU down-blend cylinders (red data points). The onesigma error bars reflect only the statistical counting uncertainty in the assay enrichment. 


\subsection{Non-Traditional Assay}

Figure 13 illustrates the assay results for the high-energy gamma-ray signature for the same 26cylinder population used in the traditional analysis. A linear calibration based on the backgroundsubtracted count rate in a high-energy window spanning from 3.0 MeV to $8.0 \mathrm{MeV}$ was used to calculate the assay value for absolute ${ }^{235} \mathrm{U}$ mass. The high degree of linearity in the assay and declared values for ${ }^{235} \mathrm{U}$ mass supports the hypothesis of a predictable ${ }^{234} \mathrm{U} /{ }^{235} \mathrm{U}$ ratio for this cylinder population, even though the calibration was produced from cylinders produced by multiple vendors and is therefore not "facility-specific." The relative standard deviation of the assay relative to the declared ${ }^{235} \mathrm{U}$ mass is $3.7 \%$ for the 23 full product cylinders, 4.9\% for the two natural-enrichment cylinders and $32 \%$ for the single depleted cylinder.

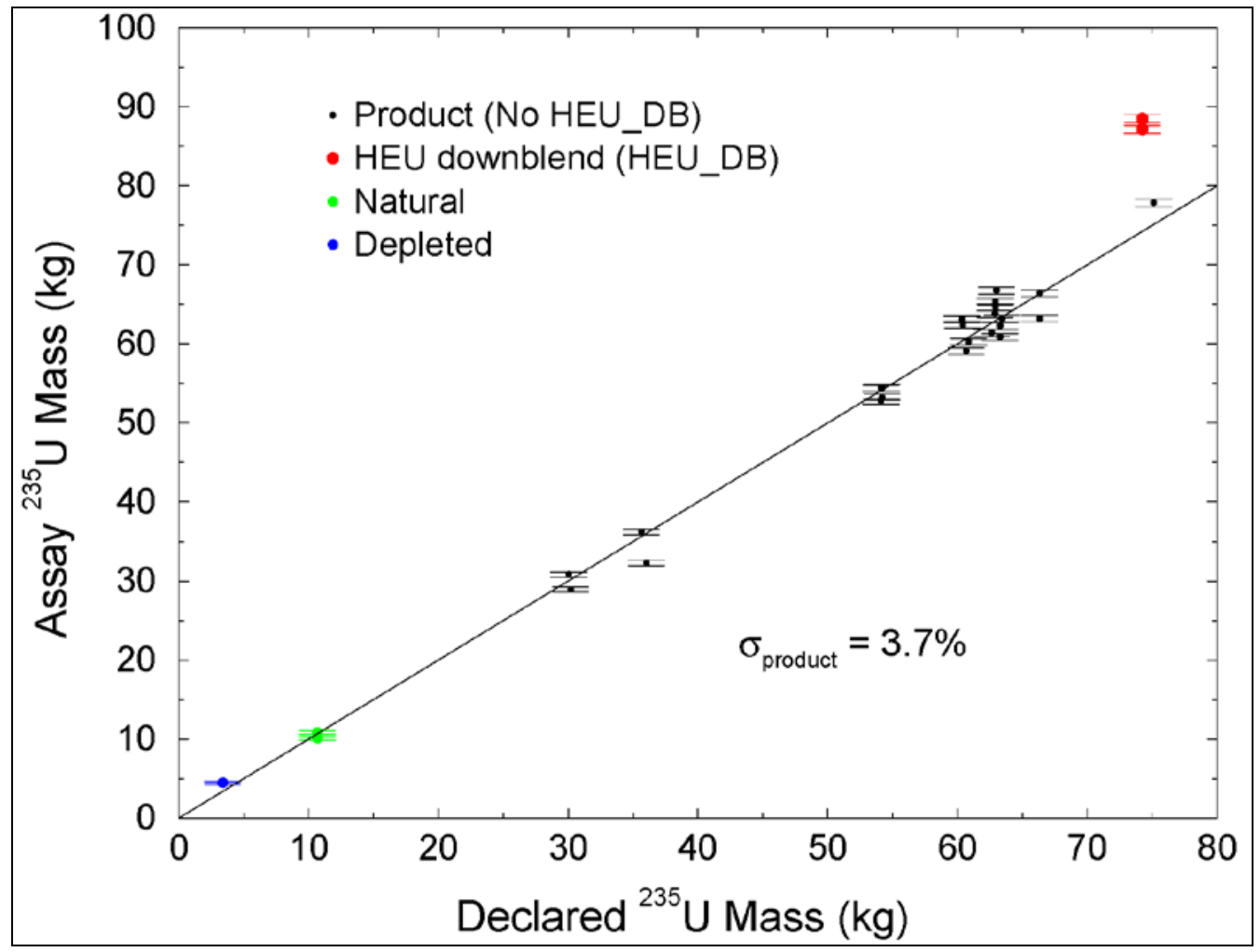

Figure 13. Assay values for ${ }^{235} \mathrm{U}$ mass versus declared ${ }^{235} \mathrm{U}$ mass using the non-traditional high-energy gamma-ray signature. HEU downblend cylinders are plotted but are not included in the uncertainty estimates: $3.7 \%$ for product cylinders and $4.9 \%$ for natural enrichment. The nontraditional signature interrogates the entire cylinder volume and assays absolute ${ }^{235} \mathrm{U}$ mass, rather than a localized, relative enrichment level.

As described in Section 2.2, there is an approximately constant neutron emission contribution from ${ }^{238} \mathrm{U}$ over product cylinder enrichments to $5 \mathrm{wt} \%{ }^{235} \mathrm{U}$. We can estimate this contribution from the zero offset of the calibration line that is used to translate the high-energy count rate versus declared ${ }^{235} \mathrm{U}$ mass. A ${ }^{238} \mathrm{U}$ contribution to the non-traditional signature for each cylinder can then be estimated using the total declared $\mathrm{UF}_{6}$ mass, a quantity that is given in the vendor's cylinder declarations. For example, a cylinder filled to $30 \%$ of the weight limit will have ${ }^{238} \mathrm{U}$ neutron emissions that are approximately $30 \%$ of a full cylinder. To first order, this ${ }^{238} \mathrm{U}$ correction can be invariant with enrichment level because ${ }^{238} \mathrm{U}$ is at least 
95\% of the declared uranium mass (for enrichment of 5\%). We completed proof-of-principle calculations for the removal of the ${ }^{238} \mathrm{U}$ spontaneous fission source term in FY10 and were reasonably effective at achieving accurate estimates of ${ }^{235} \mathrm{U}$ mass in the four partially-filled cylinders measured during the April, 2010 AREVA measurements. These results support the assertion that a neutron-based non-traditional signature can achieve true full-volume assay and can therefore be sensitive to both material diversion scenarios and $\mathrm{UF}_{6}$ heterogeneity (e.g., in tails cylinders). This very preliminary investigation will be continued in FY11.

\subsection{Hybrid Assay}

Figure 14 provides a graphical comparison of non-traditional and traditional assay results for the 26cylinder population. Previous analysis has indicated that there is a low degree of correlation in the measurement uncertainties of the traditional and non-traditional methods, presumably because the traditional uncertainty is dominated by wall thickness variations and continuum subtraction errors while the non-traditional uncertainty is dominated by variation in the ${ }^{234} \mathrm{U} /{ }^{235} \mathrm{U}$ ratio [Smith In press]. For the 26-cylinder data set analyzed in this work, the measurement uncertainty data confirm that previous result and show a low degree of correlation (normalized correlation value of 0.049 where -1.0 is perfectly anticorrelated and 1.0 is perfectly correlated).

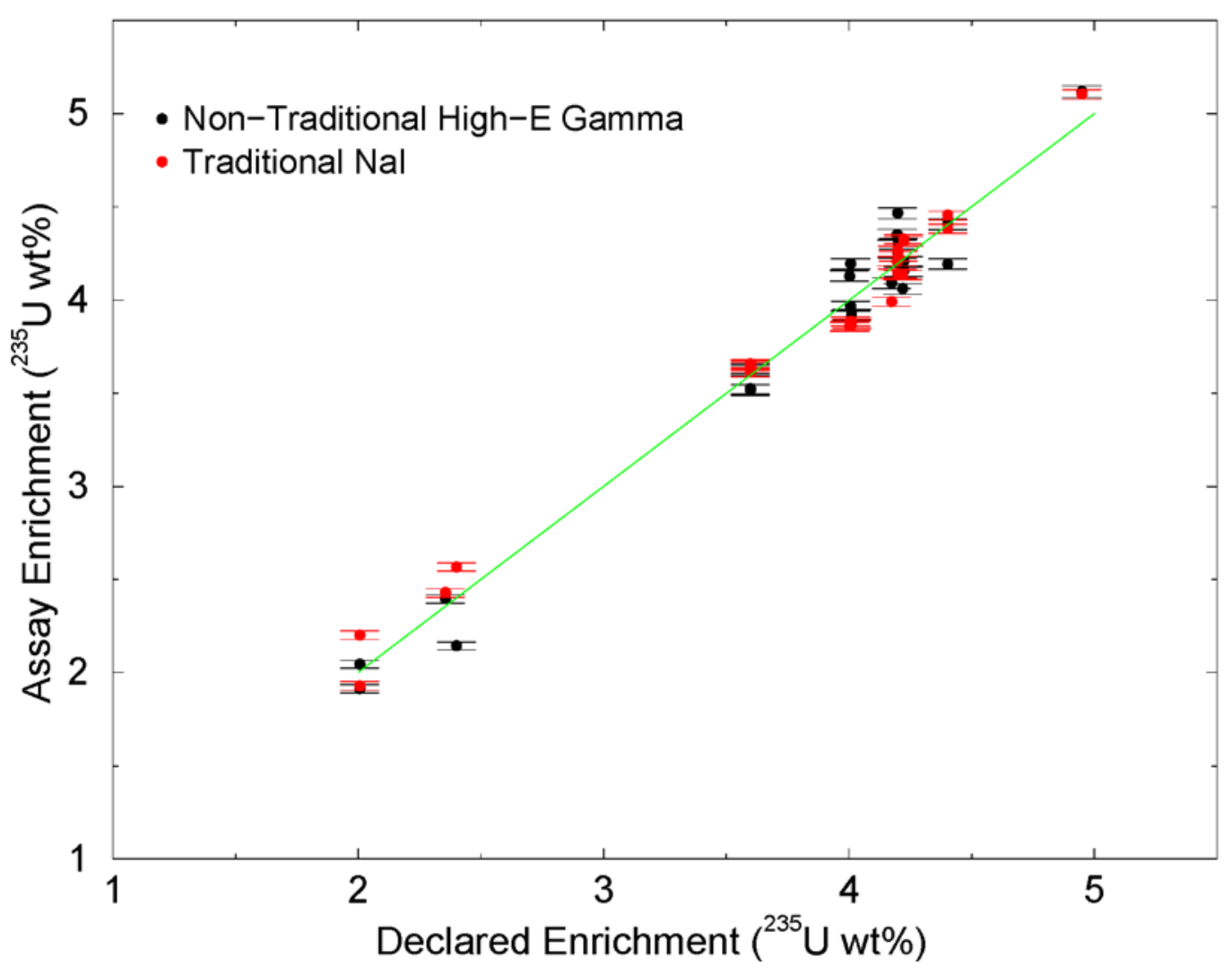

Figure 14. Comparison of non-traditional (high-energy gamma) and traditional (186-keV) assay of 26 cylinders over a range of ${ }^{235} \mathrm{U}$ enrichment. The standard deviations of the non-traditional and traditional assay of product cylinders are $3.7 \%$ and $3.5 \%$, respectively. 
To evaluate the potential viability of a hybrid method, we combined the traditional (3.5\% uncertainty) and non-traditional (3.7\% uncertainty) assay results using a simple average. (Equal weighting is a logical starting point for the hybrid method, but more sophisticated weighting schemes may prove advantageous in terms of accuracy "balance" across product, feed and tail enrichments; see Future Work, Section 9.0.) Using this equal weighting, the relative standard deviations for the hybrid method are: $2.5 \%$ for the 23 product cylinders; $4.6 \%$ for the two natural-enrichment cylinders; $9.7 \%$ for the single depleted cylinder. Each of these uncertainties is an improvement over either the traditional or non-traditional method acting independently, and all three values are below the IAEA target uncertainties for the respective enrichment categories.

\subsection{Results Summary}

Table 2 offers a summary of the results for the traditional, non-traditional and hybrid enrichment analyses, and compares it to AREVA's assay using a fixed-geometry HPGe system and the traditional enrichment-meter method. The AREVA assay system consists of a well-collimated HPGe spectrometer ( $\sim 15 \%$ relative efficiency) positioned approximately $5 \mathrm{~cm}$ from the outer wall of 30B cylinders (see Figure 15). This fixed-geometry system is calibrated with known standards prior to cylinder measurements, and is cryogenically cooled. The enrichment analysis is based solely on the $186-\mathrm{keV}$ peak intensity. The AREVA cylinder verification system is an important point of comparison because it represents best-case performance for an attended (due to need for cryogenic cooling) NDA system. Achieving comparable or improved performance with the hybrid methods, suitable for an unattended system, would be highly desirable. 


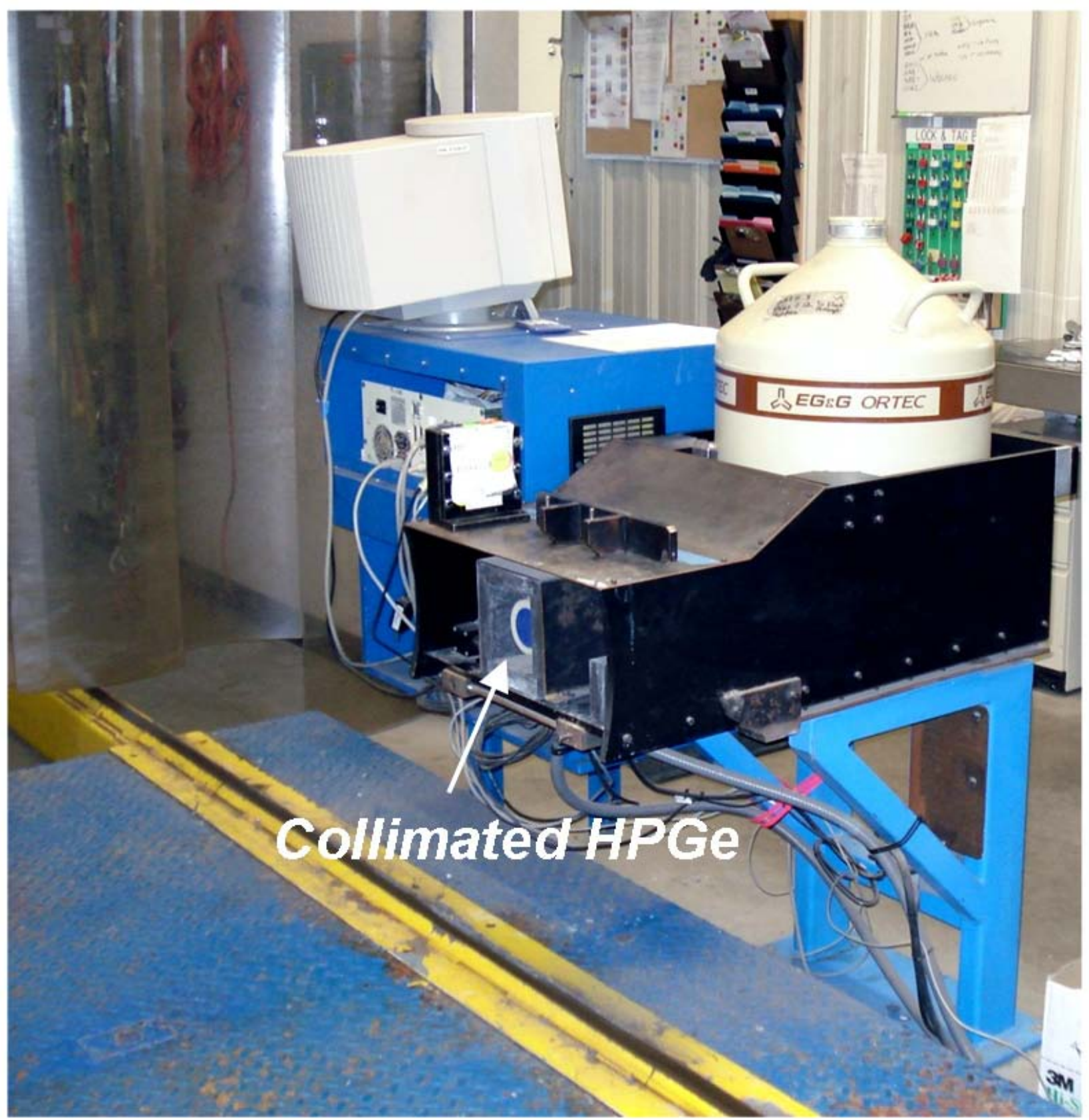

Courtesy of AREVA NP, Richland, WA

Figure 15. Photograph of AREVA's attended cylinder verification station, consisting of a wellcollimated HPGe spectrometer in a fixed gantry geometry 
Table 2. Summary of enrichment assay results for a population of 26 Type 30B cylinders: 23 product (greater than $2.0 \mathrm{wt} \%{ }^{235} \mathrm{U}$ ), 2 natural, and 1 depleted. Results are given in terms of relative standard deviation from the declared enrichment values.

\begin{tabular}{|l|c|c|c|}
\hline \multicolumn{1}{|c|}{ Method } & Product & Natural & Depleted \\
\hline Approximate Target Uncertainty [Aigner 2001] $^{1}$ & $\mathbf{5}$ & $\mathbf{1 0}$ & $\mathbf{1 5}$ \\
\hline Traditional HPGe (from AREVA) & $\mathbf{3 . 3}$ & $\mathbf{5 . 2}$ & $\mathbf{1 2}$ \\
\hline Traditional LaBr (SWC method) & 3.1 & 9.4 & 11 \\
\hline Traditional NaI (SWC method) & 3.5 & 7.9 & 13 \\
\hline Non-Traditional NaI & 3.7 & 4.9 & 32 \\
\hline Hybrid NaI (simple average) & $\mathbf{2 . 5}$ & $\mathbf{4 . 6}$ & $\mathbf{9 . 7}$ \\
\hline
\end{tabular}

${ }^{1}$ The target uncertainties are estimated from the combination of systematic and random errors given in Kuhn and should be used only to set the scale of the target uncertainties. 


\subsection{Interim Conclusions: Viability of Hybrid Assay Methods}

Traditional enrichment-meter assay using specially collimated NaI spectrometers and a SquareWave-Convolute algorithm can achieve uncertainties comparable to HPGe and LaBr for product, natural and depleted cylinders. The SWC algorithm used in this work, though first-generation, effectively extracted the net count rate in the 186-keV peak by removing both constant and linear components of the underlying continuum — a large source of uncertainty in the traditional enrichment-meter approach. Testing indicated that the SWC algorithm will be relatively insensitive to variations in energy resolution and gain drift in an unattended monitoring scenario.

Non-traditional signatures measured using NaI spectrometers enable interrogation of the entire cylinder volume and accurate measurement of absolute ${ }^{235} U$ mass in product, natural and depleted cylinders. This work confirms and expands prior work by the authors on the viability of the nontraditional high-energy gamma-ray signatures and the idea of neutron detection without dedicated neutron detectors. Specifically designed iron/polyethylene collimators and neutron reflectors provided a significant increase in the intensity of this high-energy signature. Results presented here indicate that, even for cylinders from multiple vendors/facilities, the stability of the ${ }^{234} U /{ }^{235} U$ ratio is sufficient to make the non-traditional metric viable for material ranging from 0.2 to $5 \mathrm{wt} \%{ }^{235} \mathrm{U}$, under assumptions of orebased feed.

A hybrid enrichment assay method can achieve lower uncertainties than either the traditional or nontraditional methods acting independently, and provides a number of operational advantages. There is a low degree of correlation in the systematic errors of the two individual methods because the primary sources of inaccuracy are different for each. Consequently, their combination results in lower overall uncertainties.

This work has indicated that the hybrid NDA method has the potential to serve as the foundation for an unattended cylinder verification station. When compared to today's handheld cylinder verification approach, such a station would have the following advantages:

- Improved enrichment assay accuracy for product, tail and feed cylinders

- Full-volume assay of absolute ${ }^{235} \mathrm{U}$ mass

- Assay of minor isotopes $\left({ }^{234} \mathrm{U}\right.$ and $\left.{ }^{232} \mathrm{U}\right)$ important to verification of feedstock origin

- Single instrumentation design for both Type 30B and Type 48 cylinders

- Redundancy: If one of the NaI sensors in the array fails, the system can remain operational

- Instrumentation (NaI) and algorithms that are suitable for long-term unattended operation

- Substantial reduction in the inspector manpower associated with cylinder verification

When compared to an assay approach using only a non-traditional neutron-based signature (i.e., totals neutron counting, not to be confused with the coincidence neutron approach that is capable of direct ${ }^{235} \mathrm{U}$ quantification [Menlove In Press]), the hybrid assay methods could offer the following advantages: 
- Improved resistance to spoofing scenarios. For example, a totals-neutron assay method (i.e., indirect ${ }^{235} \mathrm{U}$ assay) could be spoofed using inert material in the cylinder to achieve the declared cylinder mass and a neutron source of appropriate size. The hybrid method would detect such a diversion scenario, on account of the direct (traditional) ${ }^{235} \mathrm{U}$ measurement.

- Improved detection of anomalous feed material. If the non-traditional signature intensity is not consistent with the direct ${ }^{235} \mathrm{U}$ (traditional) signature, it could be an indication that the feedstock to the enrichment plant is different than declared (or expected), or that process conditions have changed in a way that warrants further investigation. 


\subsection{Modeling for Cylinder NDA Development}

Researchers have used modeling of the $\mathrm{UF}_{6}$ cylinder assay scenario to support prototype design choices and for enrichment algorithm development. For example, simulation can quickly explore the impact of different spectrometer collimation and shielding configurations, but it would be timeconsuming and expensive to do so in the field. One could also use simulation to generate large libraries of "virtual assay signatures" to support algorithm development, over the large parameter space important to a viable instrument (e.g., enrichment levels, ${ }^{232} \mathrm{U}$ contamination levels and wall-thickness variation).

\subsection{MCNP Models}

We developed MCNP5 models for five independent source terms: ${ }^{235} \mathrm{U}$ photons, ${ }^{238} \mathrm{U}$ photons, ${ }^{232} \mathrm{U}$ photons, ${ }^{234} \mathrm{U}(\alpha, n)$ neutrons and ${ }^{238} \mathrm{U}$ spontaneous fission neutrons, using an assumed decay time of 6 months for the generation of photon source terms. This is a potential source of bias between modeling and measurement for the ${ }^{234 \mathrm{~m}} \mathrm{~Pa}$ lines, since most of the cylinders measured in the FY-2010 AREVA campaigns were only 3-4 months old, and ${ }^{238} \mathrm{U} /{ }^{234 \mathrm{~m}} \mathrm{~Pa}$ are not in equilibrium until almost 6 months. The MCNP5 transport calculations included the characteristic gamma rays from the various uranium isotopes and their daughters and neutron-induced gamma rays (including capture and inelastic scattering in the iron walls and $\mathrm{UF}_{6}$ volume itself). We ignored the decay of fission products from spontaneous and induced fission, as well as decay of the ${ }^{22} \mathrm{Na}$ formed from ${ }^{19} \mathrm{~F}(\alpha, \mathrm{n})$ reactions. Each of the five MCNP5 models produced a "basis vector" for the specific spectral contribution from that source term (e.g., ${ }^{238} \mathrm{U}$ and its daughters), on a per-gram basis. We then combined those basis vectors in appropriate intensities to create a virtual cylinder of specific enrichment (e.g., 4.95 weight $\%{ }^{235} \mathrm{U}$ ) and minor isotope concentration (e.g., 200 parts per trillion ${ }^{232} \mathrm{U}$ ). Below we describe the development of the MCNP models used to create the uranium isotope basis vectors.

The measurement scenario modeled in MCNP (see Figure 16) consists of an iron cylinder used to approximate a Type 30B shipping container. The simulated 30B has a diameter of $76.2 \mathrm{~cm}$ (30") with a $1.27 \mathrm{~cm}(0.5$ ”) thick wall. The simulation differs from the actual geometry by having flat, rather than round, end caps. We adjusted the length of the simulated cylinder to preserve the internal cylinder volume of an actual 30B. $\mathrm{UF}_{6}$ occupies the lower $60 \%$ of the cylinder volume with a nitrogen atmosphere filling the rest. The cylinder rests on a cart that crudely approximates the AREVA trolley in terms of cross-sectional dimensions, but no effort was made to achieve the proper amount or geometry of iron in the simulated cylinder, a deficiency that could contribute a negative bias in the predicted intensity of the non-traditional signatures.

The detector array consists of the three gamma-ray spectrometers described previously: $7.6 \times 7.6 \mathrm{~cm}$ NaI, a $3.8 \times 3.8 \mathrm{~cm} \mathrm{LaBr}$, and a $5.1 \times 10.2 \times 20.3 \mathrm{~cm} \mathrm{NaI}$. The detector collimation/converter geometry is based on the prototype field system configurations used in the AREVA field measurements performed in April and May of 2010. For the April measurements, we employed the lead collimators on the $\mathrm{LaBr}$ and small NaI, while the large NaI used the Fe/poly converter; for the May measurements, we utilized the Fe/Poly collimator/converters on all three gamma-ray spectrometers. For both of the detector arrays (one on each side of the simulated cylinder), there are two $122 \times 30 \times 2.5 \mathrm{~cm}$ polyethylene panels, one located above and one below the detectors, to provide neutron reflection back into the cylinder steel wall, with the goal of increasing the intensity of the non-traditional signature. 


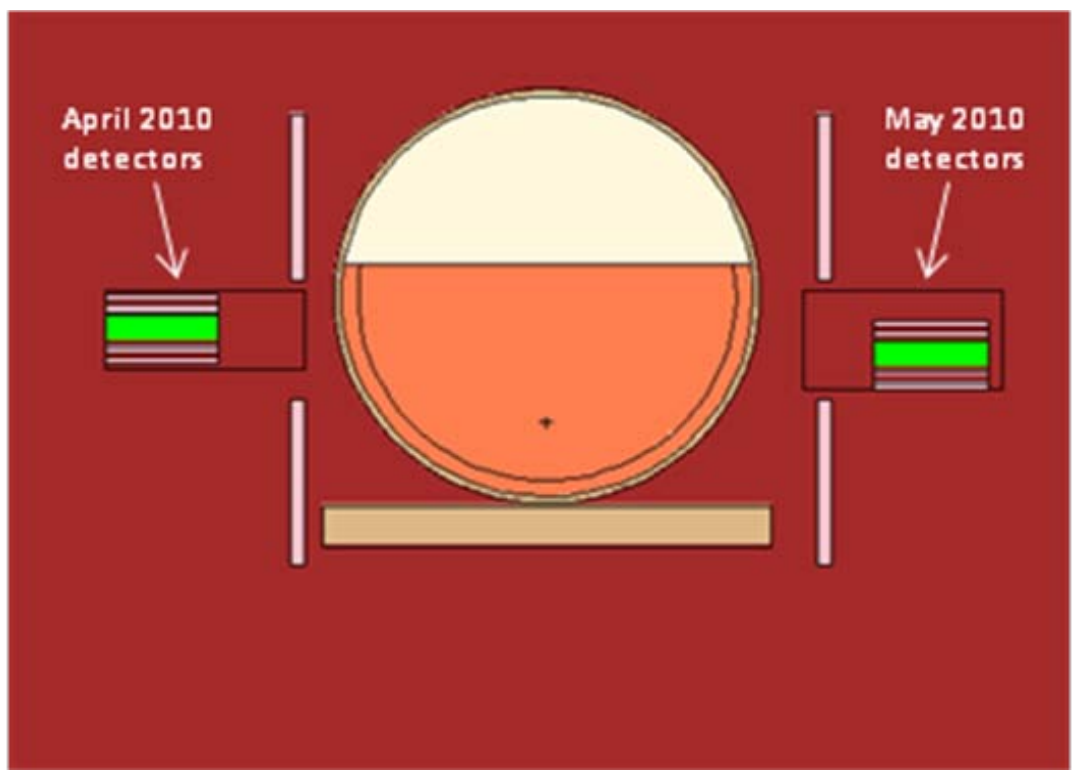

Figure 16. Cross-section of MCNP5 geometry used in the simulation of field measurements at the AREVA fuel fabrication plant in April and May of 2010

MCNP convolves the spectrometer pulse-height tallies (F8 in MCNP) with the Gaussian Energy Blurring function in MCNP to mimic the energy resolution of the specific $\mathrm{LaBr}$ and $\mathrm{NaI}$ spectrometers used in the prototype system (as described in Section 3.1). Figure 17 shows the measured data for energy resolution, along with analytic approximations for the energy-dependent resolution, G(E). We produced the analytic approximations by fitting the functional form

$$
G(E)=A+B \sqrt{E+C E^{2}}
$$

to the measured energy resolution data. The products of that fitting were values for the constants $\mathrm{A}, \mathrm{B}$ and $\mathrm{C}$ required by the GEB function in MNCP. Table 3 displays the calculated values for $\mathrm{A}, \mathrm{B}$ and $\mathrm{C}$ for each of the three gamma-ray spectrometers.

Table 3. Parameters for the MCNP GEB resolution function used in modeling the energy response of the three spectrometers deployed at AREVA in April and May 2010. We determined the parameters by fitting measured energy resolution data.

\begin{tabular}{|l|c|c|c|}
\hline \multicolumn{1}{|c|}{ Coefficient } & LaBr & NaI 7.6 $\times \mathbf{7 . 6} \mathbf{~ c m}$ & NaI 5 × 10 × 20 cm \\
\hline $\mathbf{A ~ ( M e V ) ~}$ & $4.16 \times 10^{-3}$ & $-1.06 \times 10^{-2}$ & $-1.21 \times 10^{-2}$ \\
\hline $\mathbf{B ~ ( M e V} \mathbf{1 / 2})$ & $1.30 \times 10^{-2}$ & $7.45 \times 10^{-2}$ & $8.76 \times 10^{-2}$ \\
\hline $\mathbf{C}($ dimensionless) & $3.98 \times 10^{-1}$ & $-7.22 \times 10^{-3}$ & $8.82 \times 10^{-2}$ \\
\hline
\end{tabular}




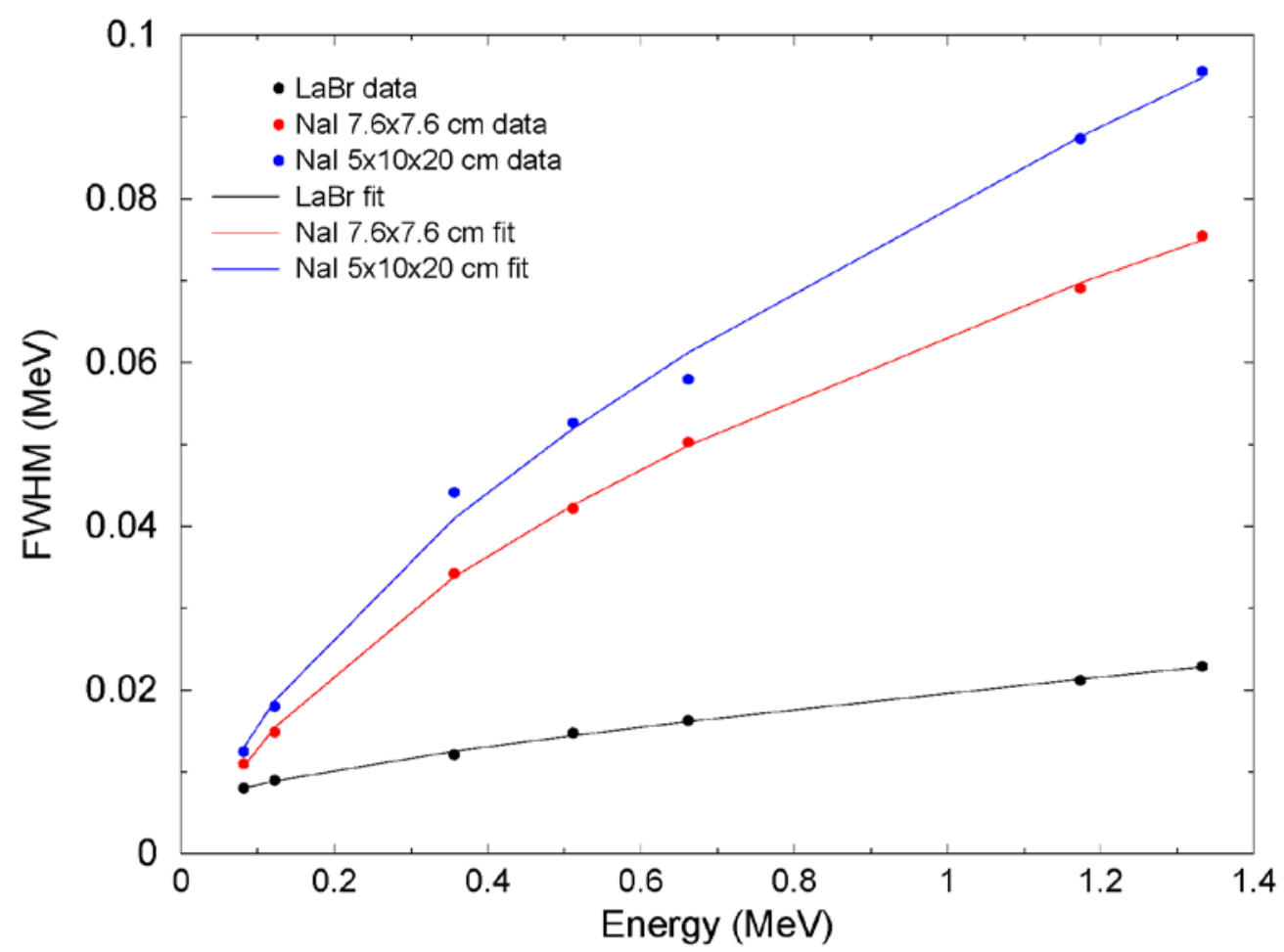

Figure 17. Measured sensor energy resolution data and fits to the data for $\mathrm{UF}_{6}$ cylinder assay portal deployed at the AREVA fuel fabrication plant in April and May 2010.

In order to improve the computational efficiency of the MCNP models used for the characteristic gamma rays emitted by ${ }^{235} \mathrm{U},{ }^{238} \mathrm{U}$ and ${ }^{232} \mathrm{U}$, we developed a variance reduction approach utilizing shells of $\mathrm{UF}_{6}$. In this approach, we confined the emitting volume to cylindrical shells with a thickness defined by the "infinite thickness" for the most prominent gamma-ray emitted by each of the uranium isotopes (and daughters): $186 \mathrm{keV}$ for ${ }^{235} \mathrm{U}, 1001 \mathrm{keV}$ for ${ }^{238} \mathrm{U}$ (from daughter ${ }^{234 \mathrm{~m}} \mathrm{~Pa}$ ) and $2614 \mathrm{keV}$ for ${ }^{232} \mathrm{U}$ (from daughter ${ }^{208} \mathrm{Tl}$ ). We estimated the infinite thickness value for each isotope using a separate MCNP5 model of comprised of a small cylinder of $\mathrm{UF}_{6}$ with tally volume near one end, as depicted in Figure 18. We varied the cylinder's length and normalized the flux tally results by the emitting volume to determine when this normalized flux intensity reached an asymptotic value. Figure 19 gives an example of those results for the ${ }^{238} \mathrm{U}$; the data indicate that a shell thickness of approximately $10 \mathrm{~cm}$ will emulate the surface intensity of 1001-keV gamma rays emitted by cylinder filled to capacity with $\mathrm{UF}_{6}$. The infinite thicknesses used for ${ }^{235} \mathrm{U},{ }^{238} \mathrm{U}$, and ${ }^{232} \mathrm{U}$ were $3 \mathrm{~cm}, 10 \mathrm{~cm}$, and $15 \mathrm{~cm}$, respectively. 


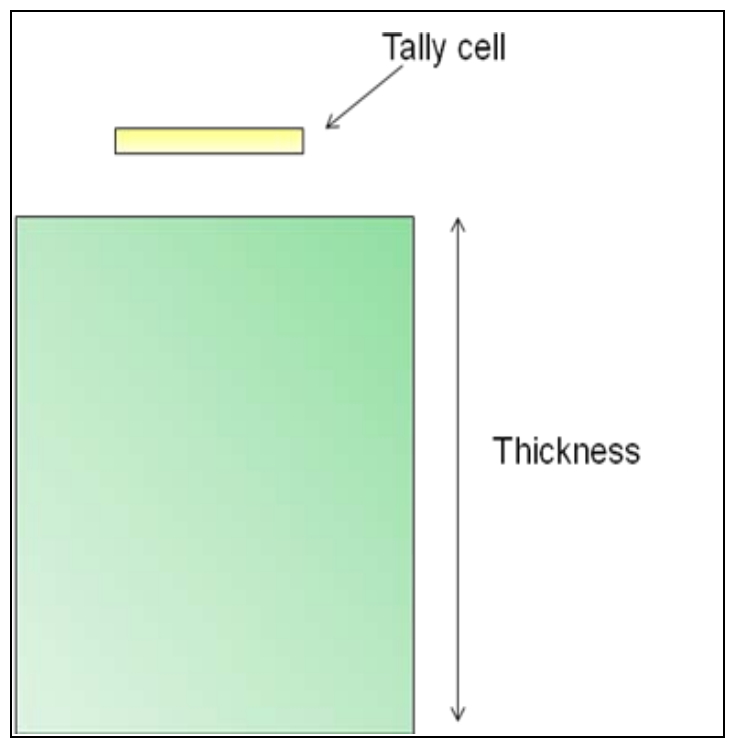

Figure 18. Schematic of the MCNP5 modeling approach used to estimate an infinite thickness for gamma-energies of 186, 1001 and $2614 \mathrm{keV}$

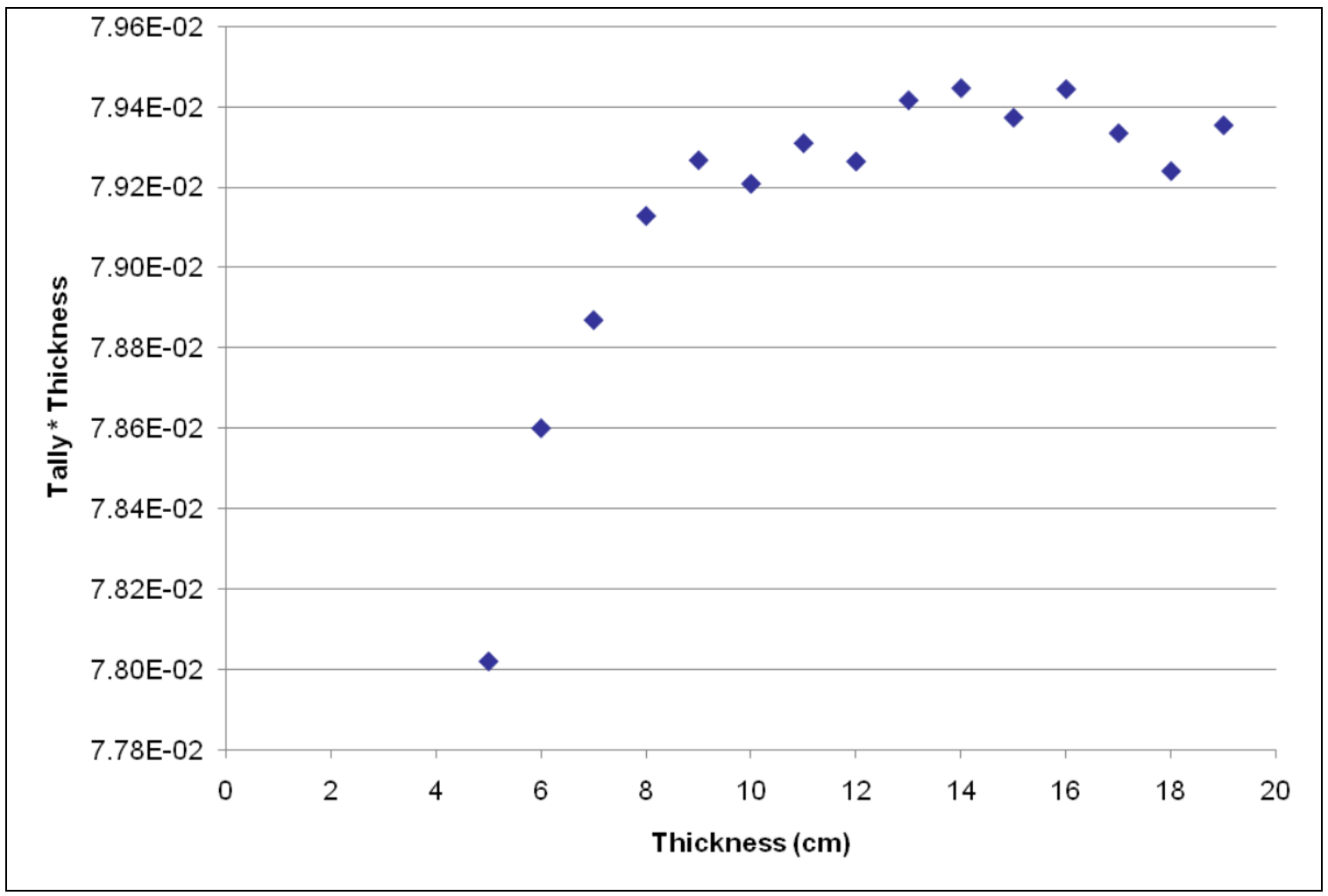

Figure 19. Example of data from infinite-thickness simulations for the $1001 \mathrm{keV}$ emitted by the ${ }^{238} \mathrm{U}$ daughter ${ }^{234 \mathrm{~m}} \mathrm{~Pa}$. Based on this data, one can approximate the gamma-ray flux at the surface of a cylinder filled to capacity with $\mathrm{UF}_{6}$ using a 10 -cm cylindrical shell. 
We then normalized the MCNP F8 tally outputs to achieve an isotope-by-isotope emission intensity in units of counts per emitted photon, per gram of source isotope. We calculated the number of photons emitted by each isotope by summing the tabulated yields (photons/decay) over all gamma-ray energies included in the MCNP source definition for that particular isotope.

The mass of each isotope in the cylinder is calculated using the declared cylinder fill level (i.e., volume of $\mathrm{UF}_{6}$ ) and enrichment value. Multiplying the tally results by the declared mass then rectifies the mass normalization. To correct for the reduced emitting volume used in the shell-based variance reduction approach, we adjusted the declared mass by the ratio of the volume of the source shell to the total fill volume of $\mathrm{UF}_{6}$ in the cylinder. Figure 20 and Eq. 1 show the geometry and calculations used in this final normalization.

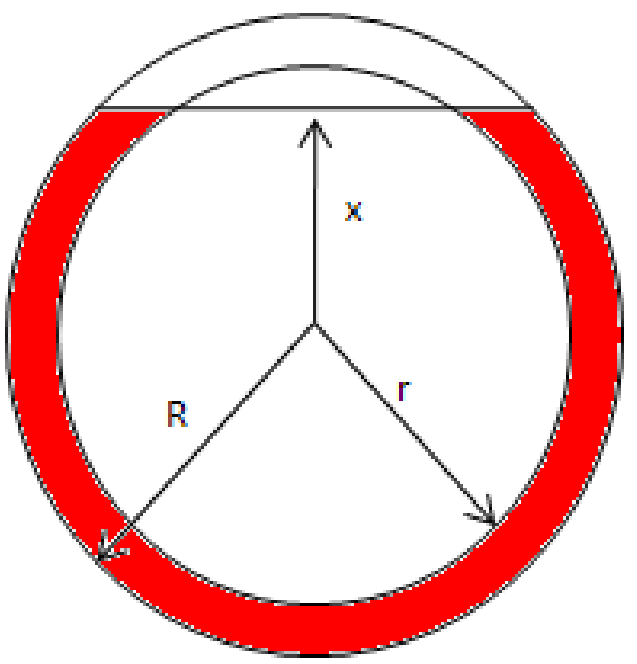

Figure 20. Schematic of the shell geometry used to reduce the variance in MCNP modeling of the $\mathrm{UF}_{6}$ cylinder assay scenario. The MCNP tally results were normalized using a volumetric mass correction utilizing the parameters $\mathrm{x}, \mathrm{r}$ and $\mathrm{R}$.

$$
C=\frac{\pi\left(R^{2}-r^{2}\right)-R^{2} \cos ^{-1}\left(\frac{X}{R}\right)+r^{2} \cos ^{-1}\left(\frac{X}{\gamma}\right)+x\left[\sqrt{R^{2}-x^{2}}-\sqrt{r^{2}-x^{2}}\right]}{\pi R^{2}-R^{2} \cos ^{-1}\left(\frac{X}{R}\right)+x \sqrt{R^{2}-x^{2}}}
$$

We only used the shell-based variance reduction approach described above for the gamma-only MCNP simulations for the characteristic emissions from ${ }^{235} U,{ }^{238} U$ and ${ }^{232} U$. We applied no variance reduction of this sort to the neutron-gamma MCNP models, however, since the neutrons are highly penetrating and neutrons emitted in the center of the $\mathrm{UF}_{6}$ volume have a high probability of escape to the outer surface of the cylinder. We created two different neutron-gamma MCNP models, one with an emitted neutron energy distribution defined for $(\alpha, n)$ reactions on ${ }^{19} \mathrm{~F}$ and one using a spontaneous fission distribution. We used the MNCP-simulated basis vector for the gamma rays produced by ${ }^{19} \mathrm{~F}(\alpha, \mathrm{n})$ reactions for both ${ }^{234} U$ and ${ }^{238} U$ alpha emission, using different neutron yield normalization based on the relative yield of neutrons given by SOURCES 4C simulations (see Section 2.2) for each isotope. We 
normalized the MCNP basis vector produced for high-energy gamma rays induced by spontaneous fission neutrons by the SOURCES 4C value for the yield (neutrons/g) of spontaneous fission neutrons in ${ }^{238} \mathrm{U}$.

The final step in preparing the simulation results for comparison with observed data is to normalize by the width of the simulated energy bin, to produce a predicted gamma-ray spectrum with units of $\mathrm{cps} / \mathrm{keV}$. Figure 21 gives examples of the simulated basis vectors and aggregate spectra for the $\mathrm{LaBr}$ and $7.6 \times 7.6 \mathrm{~cm} \mathrm{NaI}$ spectrometers viewing a cylinder of $4.4 \%$ enrichment $\left(200 \mathrm{ppt}^{232} \mathrm{U}\right)$. Figure 22 similarly illustrates basis and aggregate spectra for the $5 \times 10 \times 20 \mathrm{~cm} \mathrm{NaI}$ over an expanded energy range that spans the non-traditional signature region of interest. The following section provides examples of how the simulated spectra for cylinders with various declared enrichments and minor-isotope concentrations compared to measured spectra.
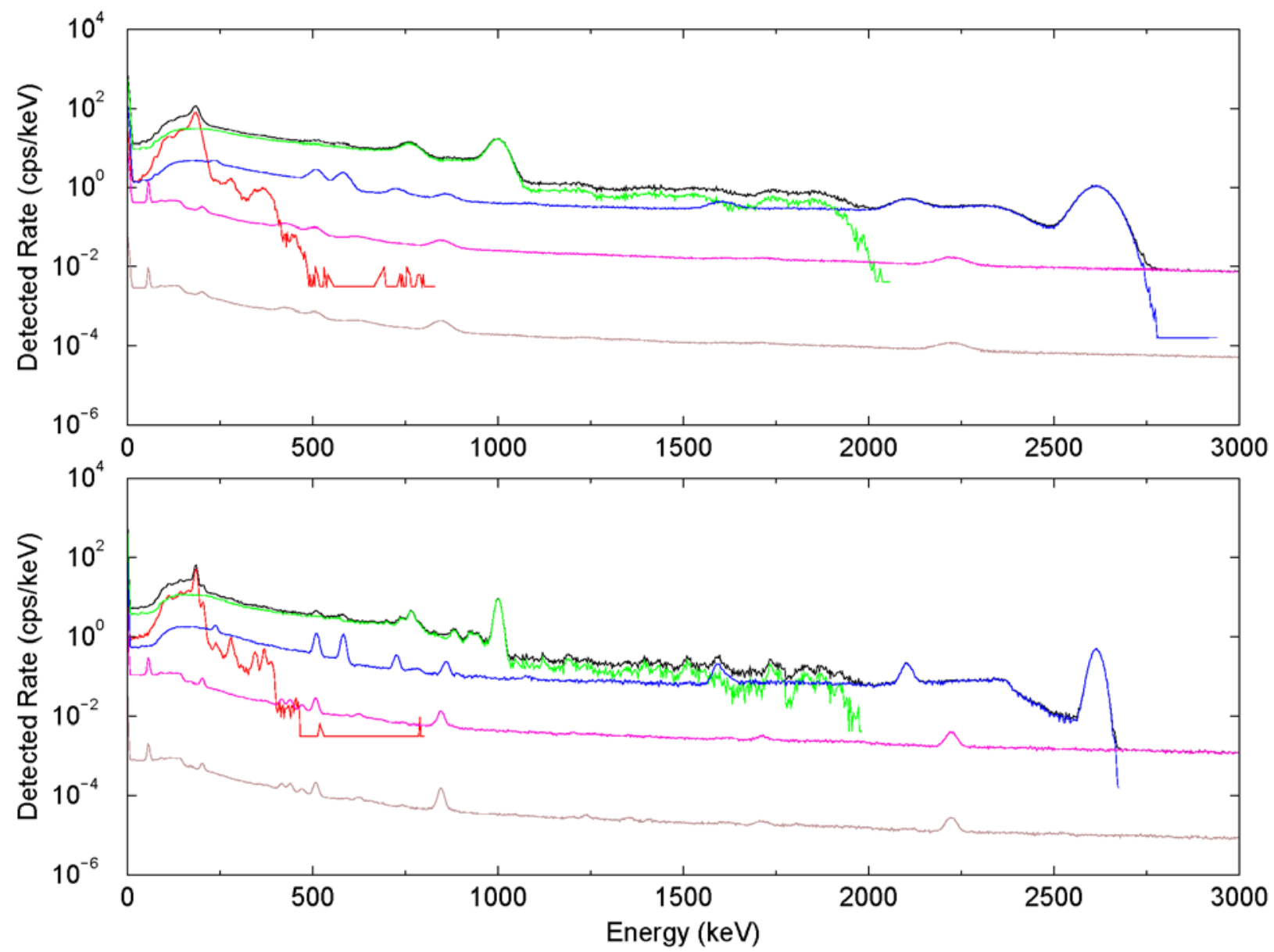

Figure 21. Examples of basis vectors simulated using MCNP5, and their summation to produce a prediction for the spectrum measured by the prototype field system. Top: $7.6 \times 7.6-\mathrm{cm} \mathrm{NaI}$. Bottom: LaBr. Black trace: Sum, 4.4\% ${ }^{235} \mathrm{U}, 200$ ppt ${ }^{232} \mathrm{U}$. Red: Uranium-235 (4.4\%). Green: Uranium-238 (95.6\%). Blue: Uranium-232 (200 ppt). Magenta: Uranium-234 ( $\alpha, n)$. Brown: Uranium-238 spontaneous fission. 


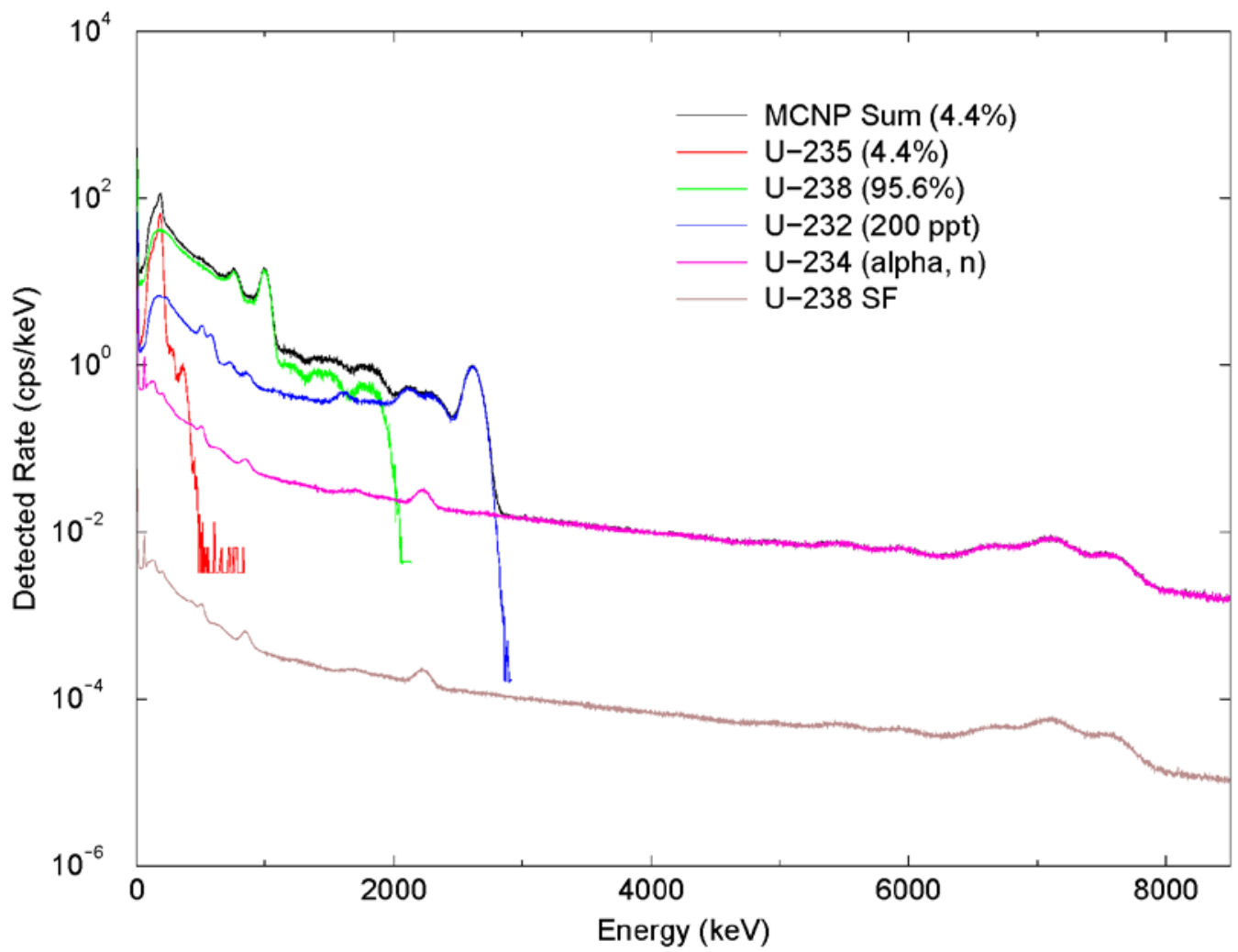

Figure 22. Examples of basis vectors for the $5 \times 10 \times 20 \mathrm{~cm} \mathrm{NaI}$ spectrometer simulated using MCNP5, and their summation to produce a prediction for the spectrum measured by the prototype field system

\subsection{Modeling Validation}

The modeling results shown here are preliminary; refining the models, continuing the validation and troubleshooting discrepancies more thoroughly will be a subject of follow-on work in FY11.

Figure 23 below gives a comparison of simulated and measured gamma-ray spectra for three different cylinders below. In those figures, reasonably good agreement (typically, within 25\%) is realized for the full-energy peak intensity, but the underlying continuum for the simulation under-predicts the measured data by roughly a factor of two. This result is consistent with discrepancies seen in previous MCNP modeling of uranium and is due to neglect of bremsstrahlung from beta-decay of isotopes in the ${ }^{238} \mathrm{U}$ decay chain. 
PNNL-19854
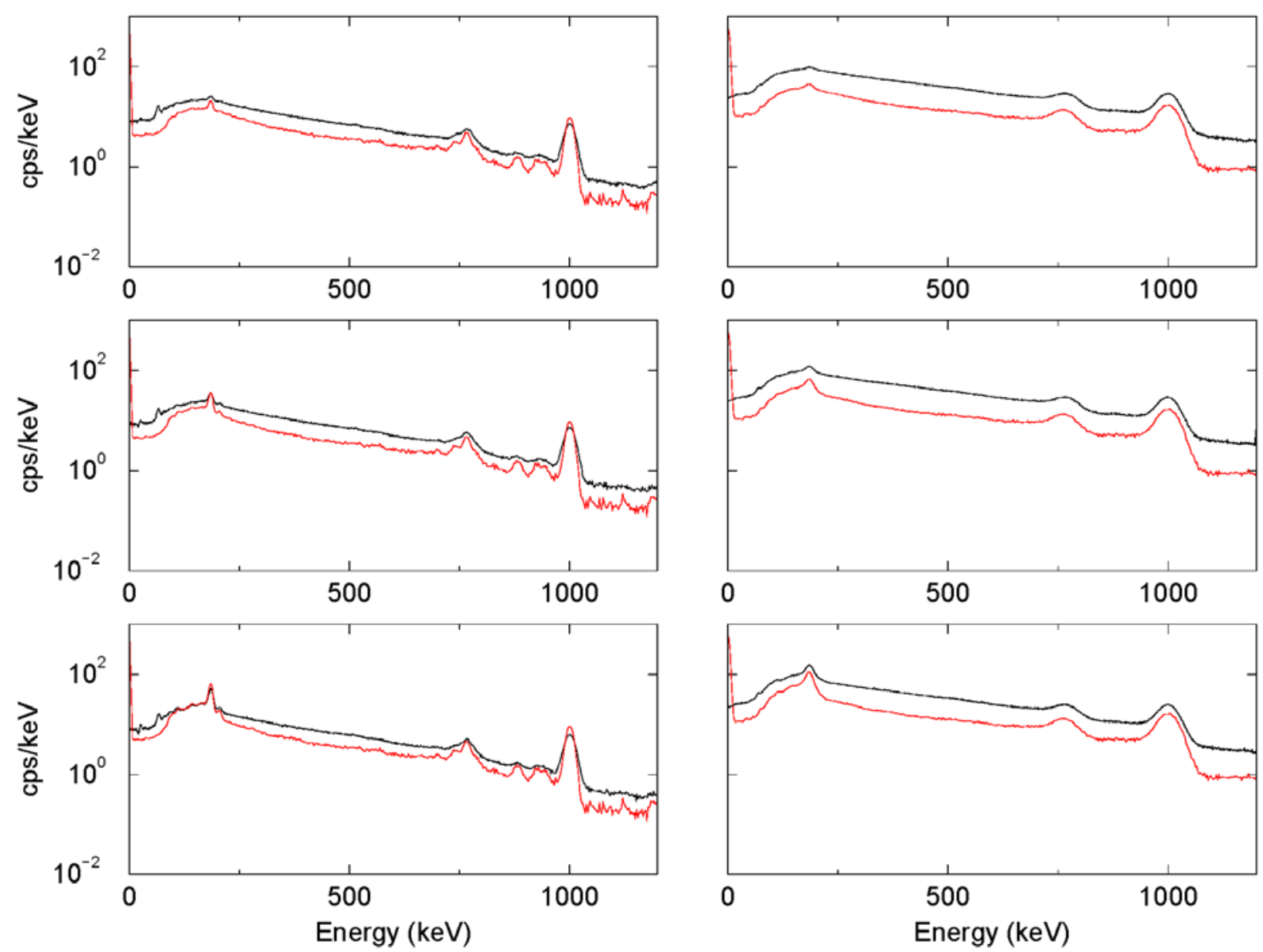

Figure 23. Comparison of measured (black curves) and simulated (red curves) spectra for the assay of cylinders of various enrichments with the $\mathrm{LaBr}$ (left) and $7.6 \times 7.6-\mathrm{cm} \mathrm{NaI}$ (right) spectrometers. Top: Natural (0.711 wt $\left.\%{ }^{235} \mathrm{U}\right)$. Middle: $2.0 \mathrm{wt} \%{ }^{235} \mathrm{U}$. Bottom: $4.4 \mathrm{wt} \%$ ${ }^{235} \mathrm{U}$.

Figure 24 provides a comparison of the simulated high-energy gamma-ray count rates, for a window of 3.0 to $8.0 \mathrm{MeV}$. 


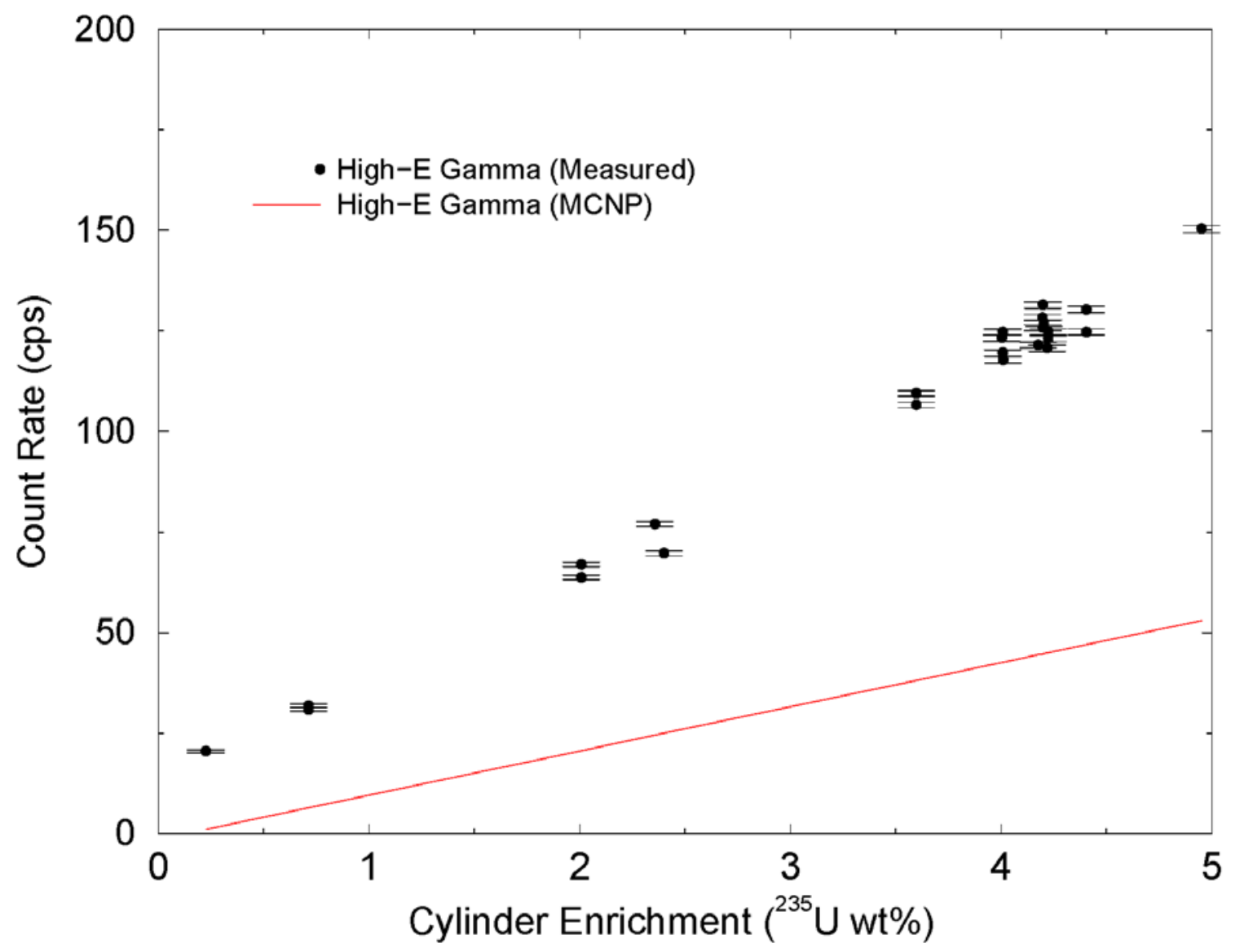

Figure 24. Comparison of measurements and simulations of the non-traditional high-energy gamma-ray signature (3.0 to $8.0 \mathrm{MeV}$ ) for a range of cylinder enrichments 


\subsection{Case Study: Non-Traditional Signature for Reactor- Recycle Feedstock}

The field measurement results presented earlier in this report demonstrated that the "non-traditional" signatures spawned from ${ }^{234} \mathrm{U}$ have the potential to be a valuable addition to an enrichment assay technique under the assumptions of ore-based feed and a facility-specific calibration. While these assumptions are likely to be valid in the vast majority of enrichment plants under IAEA safeguards, other feedstock variants may be utilized. These include uranium that has been recovered from the reprocessing of spent fuel, labeled "reactor-recycle" feed in this report, and "tails recycle" in which the depleted uranium in the tails cylinders resident at enrichment plants is processed again to wring even more of the remaining ${ }^{235} \mathrm{U}$. One driver for utilizing these alternative feedstocks is economics; for example, when the price of ore-based uranium feed is high enough that it justifies the use of alternative sources. An analysis of the driving forces and the likelihood of countries using an alternative feedstock is beyond the scope of this report. In this report, the focus is to provide preliminary data and findings on how one of these alternatives, reactor recycle uranium, might impact the utility of the signatures utilized in PNNL's hybrid enrichment approach, particularly the non-traditional signature that relies on the concentration of the minor isotope ${ }^{234} \mathrm{U}$, relative to the ${ }^{235} \mathrm{U}$ concentration (hereafter, “234/235”). The primary research questions to be addressed in this case study are:

1. How does $234 / 235$ vary with initial enrichment, burnup and cooling time for pressurized water reactor (PWR) fuels?

2. Can ${ }^{232} \mathrm{U}$, an isotope that is relatively easy to detect using the wide-range (i.e., up to $9 \mathrm{MeV}$ ) gamma-ray spectrometry utilized for hybrid assay methods, serve as a quantitative indicator of the 234/235 ratio?

The methods and preliminary findings for this case study are described in this section.

\subsection{ORIGEN-ARP for Simulating Reactor-Recycle Uranium Isotopics}

The utility of the non-traditional signatures for ${ }^{235} \mathrm{U}$ enrichment quantification in reactor-recycle uranium is dependent on the behavior of the ${ }^{234} \mathrm{U} /{ }^{235} \mathrm{U}$ ratio over the range of fuel parameters likely to be encountered with that feed type. Figure 25 shows the major production and loss mechanisms for ${ }^{234} \mathrm{U}$ in a light-water reactor. 
PNNL-19854

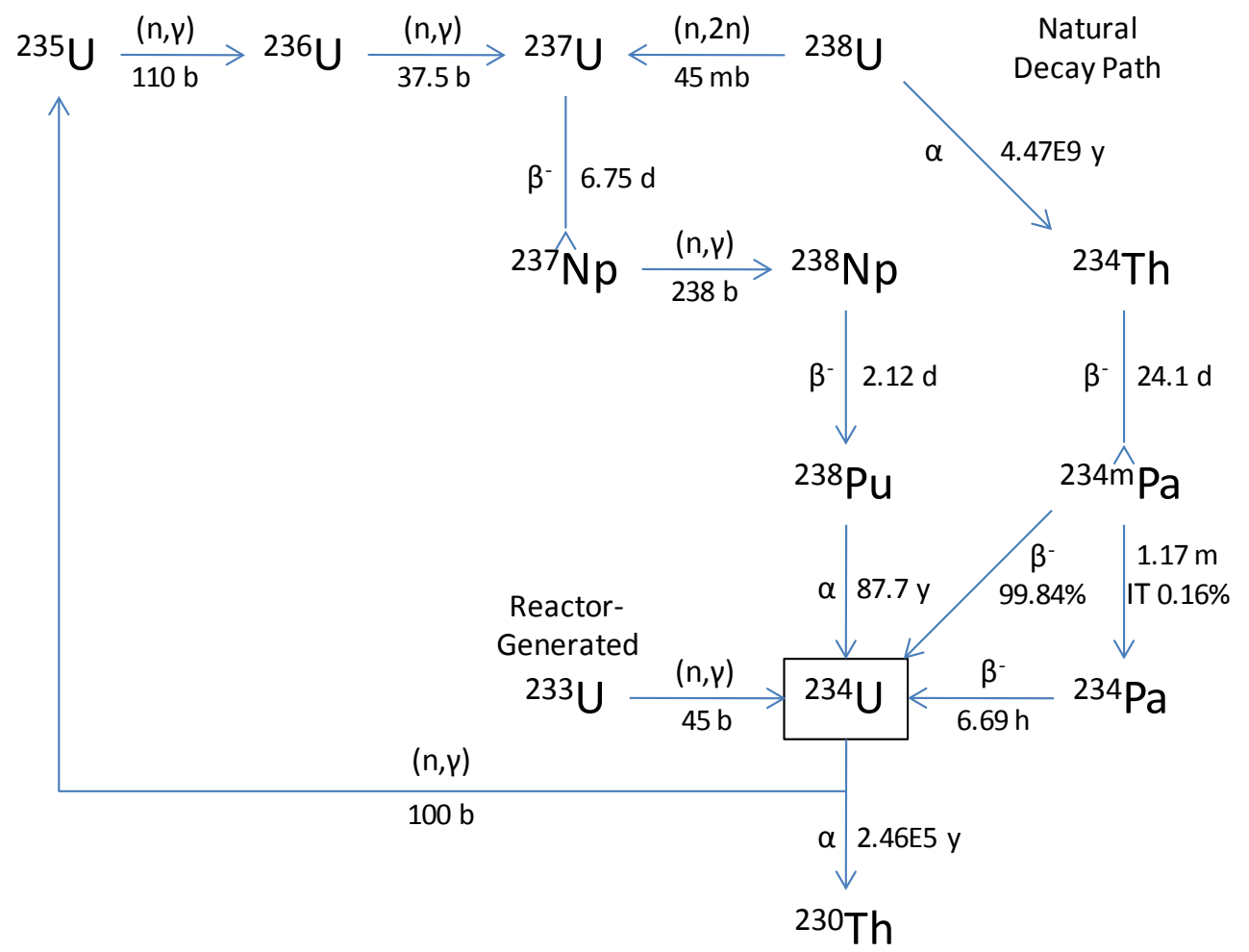

Figure 25. Select production and loss mechanisms for ${ }^{234} \mathrm{U}$ within a light-water reactor

The 234/235 ratio in spent fuel is affected by initial enrichment, burnup and cooling time (before reprocessing). In order to study these effects, we performed a series of simulations in ORIGEN-ARP [Croff 1983]. We used the Express feature in ORIGEN to model a typical PWR fuel assembly over a range of fuel parameters that might be realized in a commercial reactor: initial enrichment, burnup, and cooling time. Table 4 provides the input parameters used in the ORIGEN calculations, common to all 20 of the fuel assembly variants. Table 5 gives the range of parameters analyzed in this scoping study.

Table 4. Input parameters for ORIGEN_ARP Express modeling of PWR fuel assemblies

\begin{tabular}{|l|l|}
\hline Fuel Assembly Type: & Combustion Engineering 14 x 14 \\
\hline Average Power: & 35 MW/MTU \\
\hline Power History: & $100 \%$ up \\
\hline Uranium Basis: & $1,000,000$ grams \\
\hline Cycles: & 3 \\
\hline Libraries: & 1 per Cycle \\
\hline
\end{tabular}


PNNL-19854

Table 5. Matrix of PWR fuel parameters considered in the ORIGEN study of 234/235 variation

\begin{tabular}{|c|c|c|}
\hline Enrichments (\%) & Burnup Levels (GWd/MTU) & Cooling Times (years) \\
\hline $2,3,4,5$ & $15,30,45,60$ & $1,3,6,10,15$ \\
\hline
\end{tabular}

We adjusted the initial 234/235 for each different enrichment level in the ORIGEN fuel definition (rather than using the ORIGEN-ARP default value corresponding to natural uranium) to reflect the reality that the ${ }^{234} \mathrm{U}$ will enrich at a higher rate than the ${ }^{235} \mathrm{U}$ in the enrichment process that creates the fresh fuel. The 234/235 behavior assumed in these calculations derives from Fischer et al. [2005], as described in the Section 2.2 discussion of neutron signatures, and is confirmed by Cooley [2000]. Table 6 contains the distribution of uranium isotopes used as input to the ORIGEN-ARP calculations.

Table 6. Uranium isotopic masses (grams) in the ORIGEN-ARP fuel definitions, as a function of initial uranium enrichment

\begin{tabular}{|c|c|c|c|c|r|}
\hline $\begin{array}{c}\text { Enrichment } \\
(\mathbf{\%})\end{array}$ & U 234 & U 235 & U 236 & U 238 & Total U \\
\hline 2 & 175 & 20000 & 92 & 979733 & 1000000 \\
\hline 3 & 269 & 30000 & 138 & 969593 & 1000000 \\
\hline 4 & 364 & 40000 & 184 & 959452 & 1000000 \\
\hline 5 & 459 & 50000 & 230 & 949311 & 1000000 \\
\hline
\end{tabular}

The output of the ORIGEN calculations is a full isotopic inventory, including fission and activation products, and minor actinides. From these output files, we extracted the uranium isotopic masses and utilized them to support analyses to address the research questions defined above. Section 8.2 presents those results and findings.

\subsection{Scoping Study Results}

\subsubsection{4/235 Variation in Reactor-Recycle Uranium}

The figures below summarize the results of the ORIGEN simulations for the 234/235 variation. Those results show that 234/235 varies significantly over the fuel space considered here. The most significant variation occurs in the lower enrichment fuels where, depending on burnup and cooling time, the atomic ratio can vary between 0.0156 to 0.225 (see Figure 26), compared to the ratio in natural ore of 0.00879 . For a $2 \%$ initial enrichment, $60 \mathrm{GWd} / \mathrm{MTU}$ burnup and 15 -year cooling time, the $234 / 235$ ratio is over 30 times higher than for natural ore. The $5 \%$ fuel realized smaller variations of between 0.0114 and 0.0371 (see Table 5), which are up to four times greater than the naturally occurring ratio. 


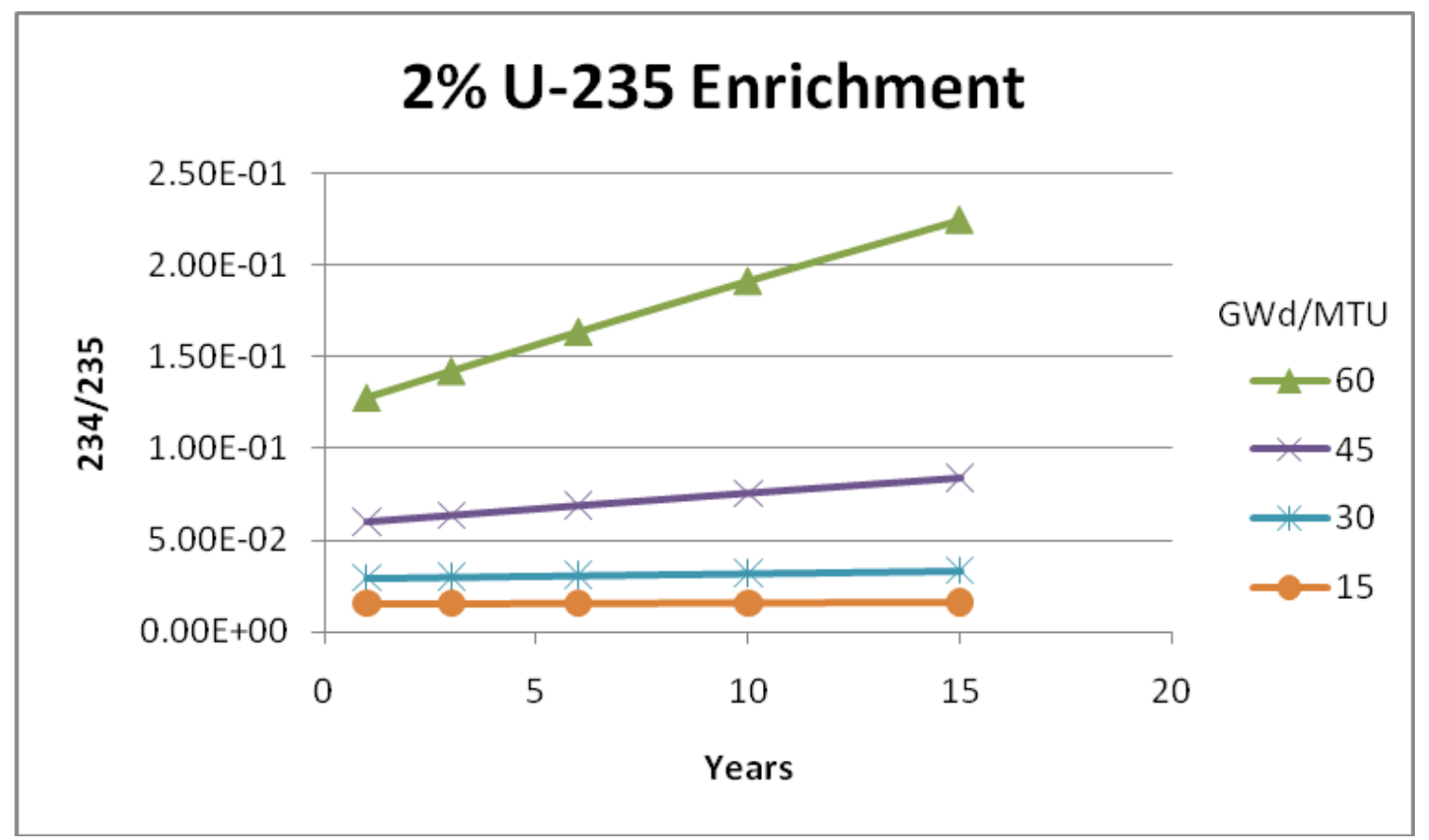

Figure 26. Uranium-234/Uranium-235 ratio for PWR fuel with an initial enrichment of $2 \mathrm{wt} \%{ }^{235} \mathrm{U}$, as a function of burnup and cooling time

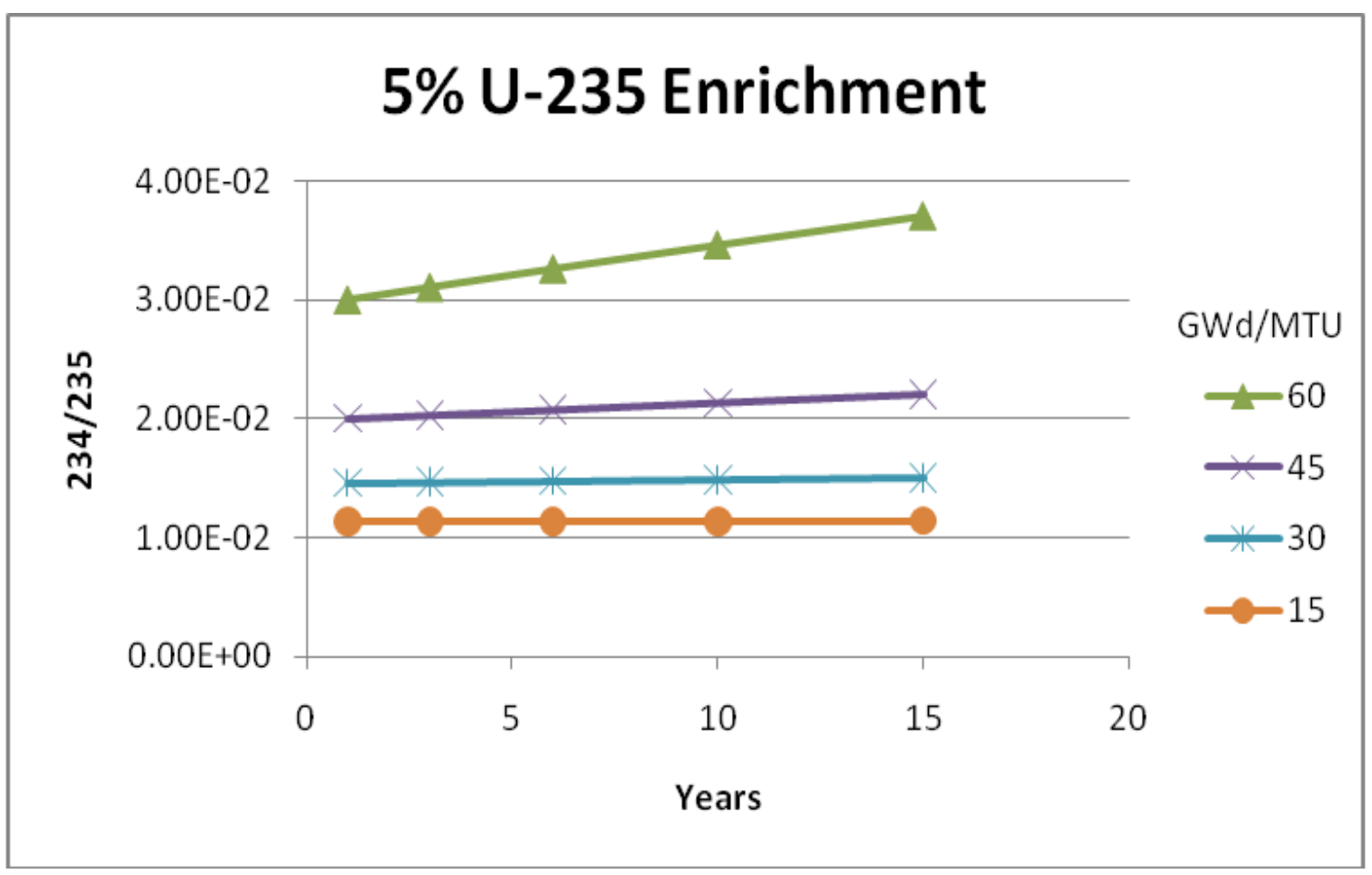

Figure 27. Uranium-234/Uranium-235 ratio for PWR fuel with an initial enrichment of 5 wt $\%{ }^{235} \mathrm{U}$, as a function of burnup and cooling time 
PNNL-19854

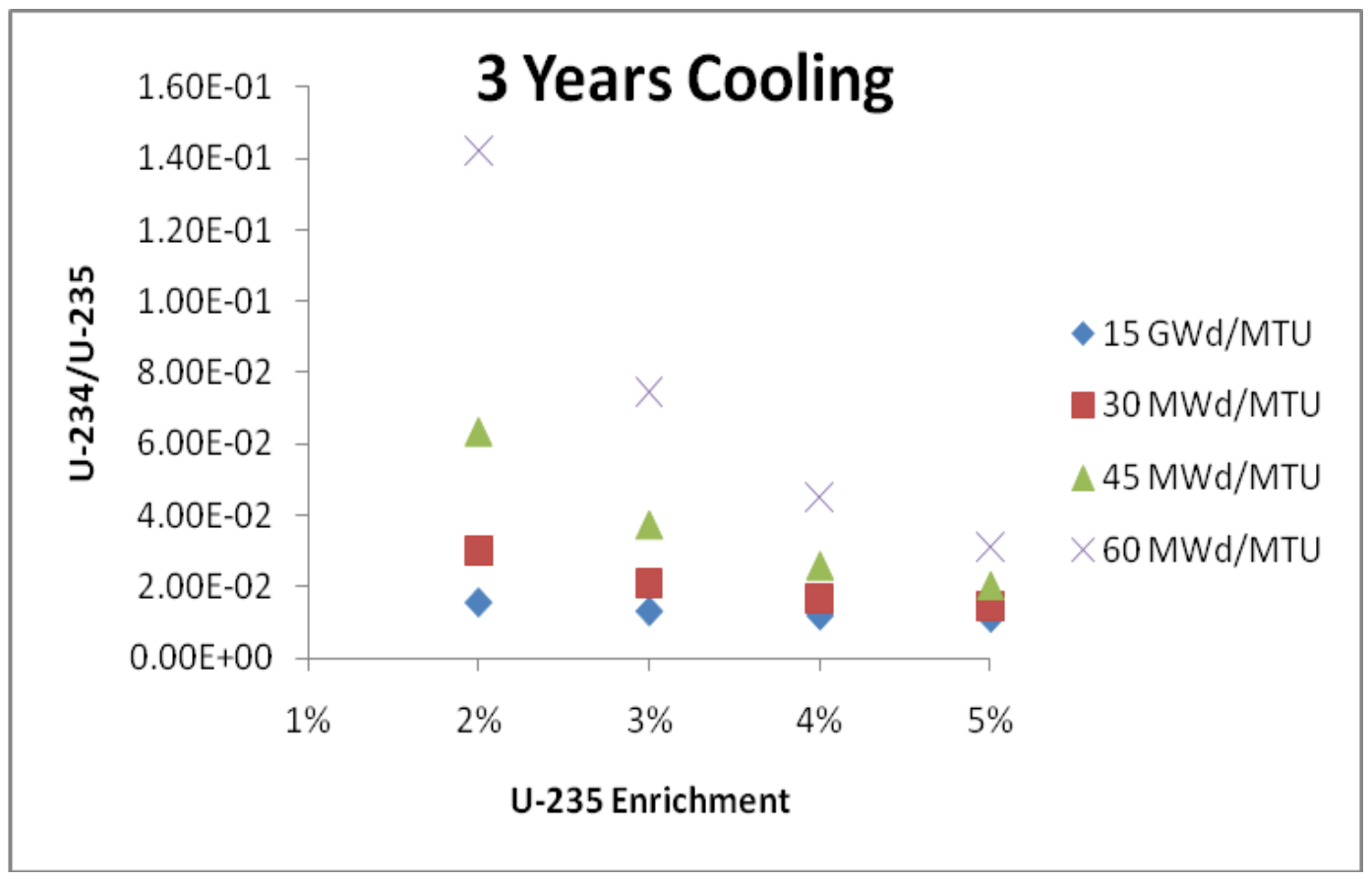

Figure 28. Uranium-234/Uranium-235 ratio for PWR fuel with a cooling time of 3 years, as a function of initial enrichment and burnup 


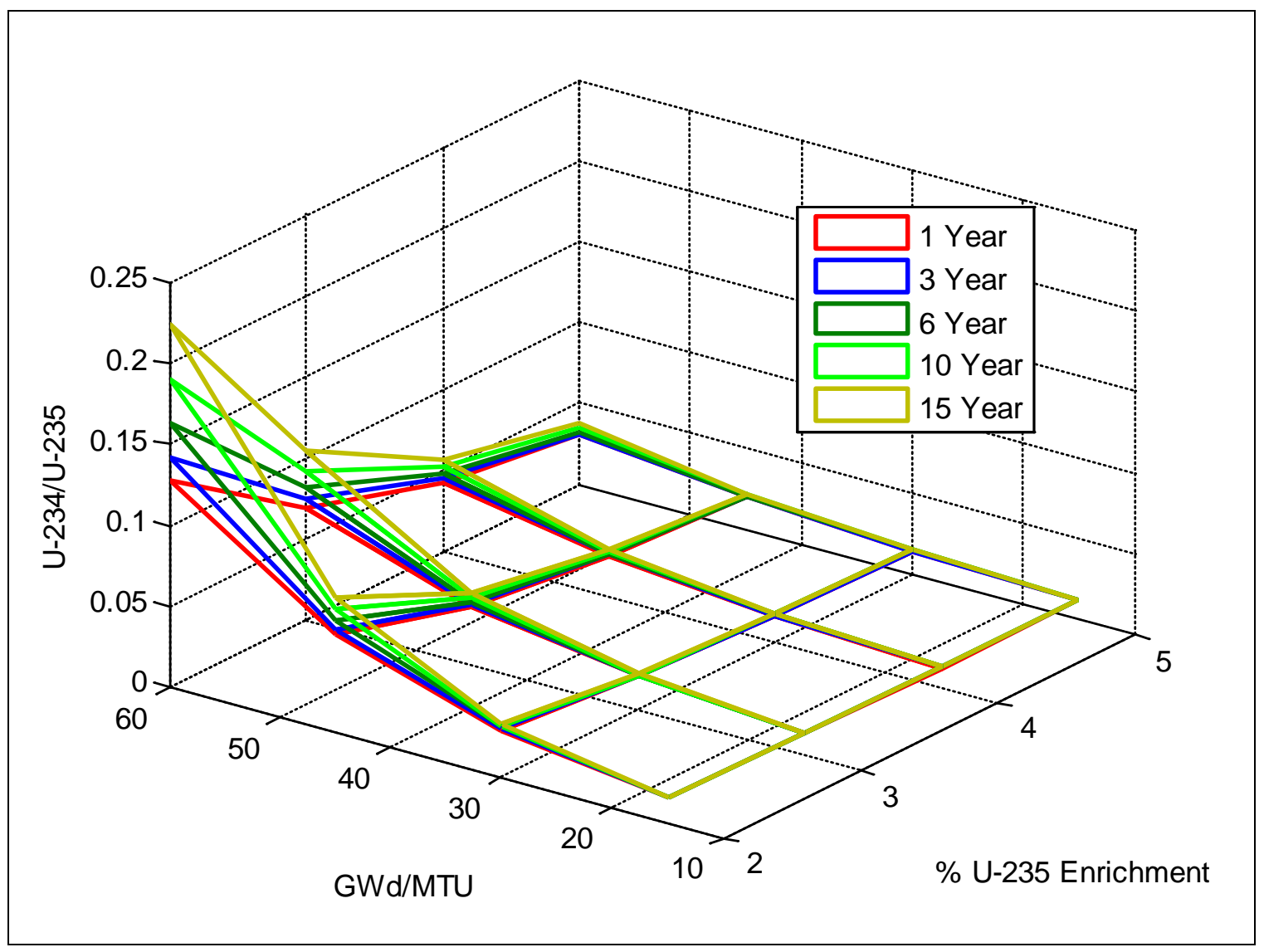

Figure 29. Uranium-234/Uranium-235 variation for PWR fuel over the range of initial enrichment, burnup and cooling time considered in this study. The maximum 234/235 value is approximately 30 times higher than the naturally occurring ratio.

\subsubsection{Uranium-232 as Quantitative Indicator of 234/235}

Uranium-232, another minor isotope of uranium, is not present in natural uranium but is created during reactor irradiation of uranium. Figure 30 illustrates the predominant production and loss mechanisms for ${ }^{232} \mathrm{U}$ in a light-water reactor[Cochran 1990]. For more discussion about its origins and utility for other radiation detection scenarios (e.g., detection of illicit nuclear material trafficking in vehicles at land border crossings), see Peurrung [1999] and Stromswold [2005]. 
PNNL-19854

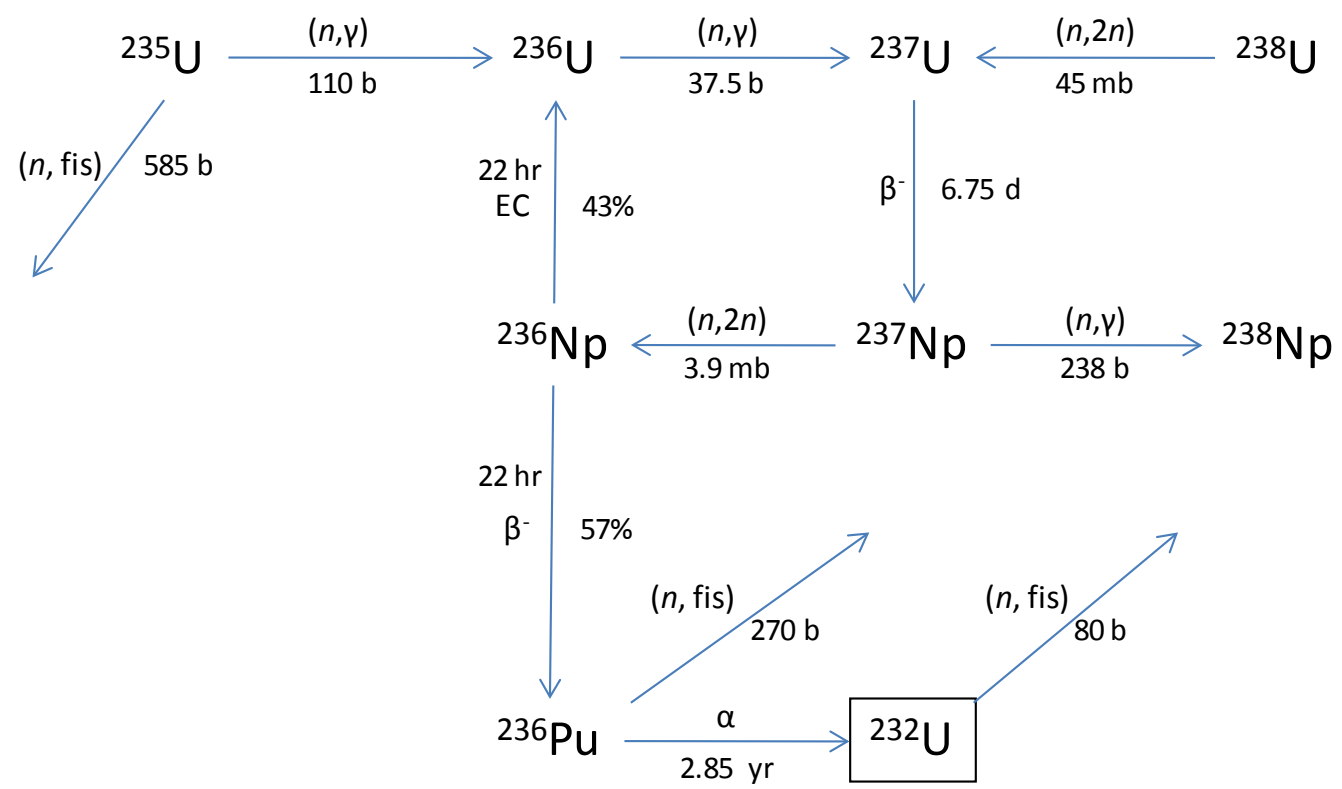

Figure 30. Production and loss pathways of ${ }^{232} \mathrm{U}$ in light-water reactor fuel [Cochran 1990]

This work explores the prospect of using ${ }^{232} \mathrm{U}$ as a quantitative indicator of the 234/235 ratio in $\mathrm{UF}_{6}$ cylinders containing reactor-recycle uranium. Figure 31 compares spectra recorded from three different $\mathrm{UF}_{6}$ cylinders, providing empirical, though qualitative, support for this concept. Two of these cylinders come from ore-based feed while the third is produced from the downblending of Russian HEU. Previous work by others has shown that Russian HEU is typically produced by enriching reactor-recycle uranium and therefore, contains elevated levels of ${ }^{232} U$, on the order of 200 ppt [Peurrung 1999]. Consequently, the expectation is that a downblended $U_{6}$ cylinder would retain some level of ${ }^{232} U$. The prominent gamma-ray signature of ${ }^{232} \mathrm{U}$ is a $2614-\mathrm{keV}$ photon emitted by the daughter ${ }^{208} \mathrm{Tl}$, and that line is clearly evident in the wide-range spectrometry utilized in the hybrid assay method. The likelihood of that daughter's gamma ray emission presenting itself in cylinders of various types is discussed later in this section. 


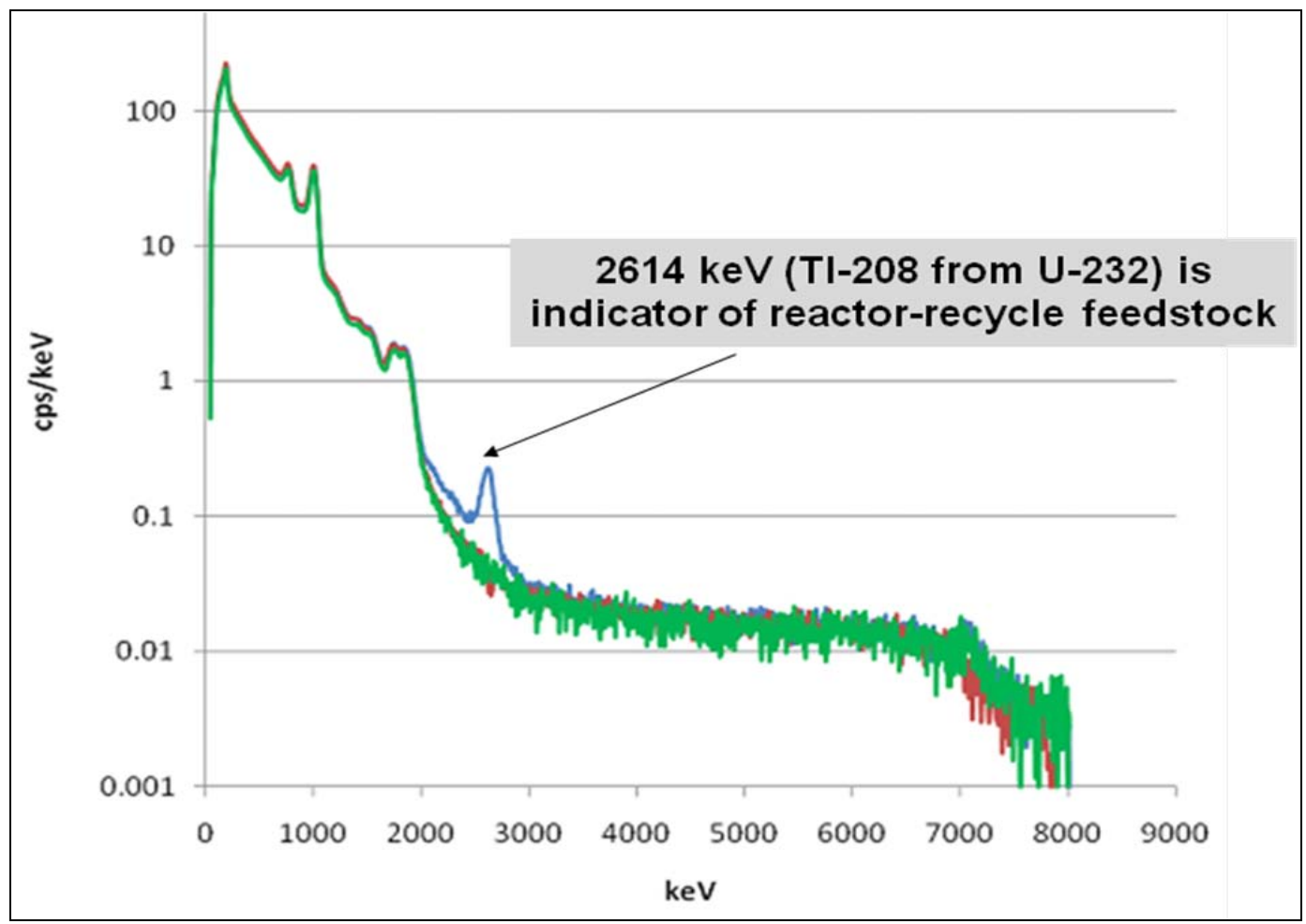

Figure 31. Gamma-ray spectra recorded from three $\mathrm{UF}_{6}$ cylinders, two of which are from ore-based feed (red and green) and the third from HEU downblend activities in Russia (blue). The presence of ${ }^{232} \mathrm{U}$ in the reactor-recycle uranium is evident from the 2614-keV gamma-ray emitted by ${ }^{208} \mathrm{Tl}$, a daughter of ${ }^{232} \mathrm{U}$.

The research question is whether this prominent and easily measured line could be used to calibrate the 234/235 behavior for reactor-recycle feed. That is, could the measured intensity of the 2614-keV peak on a cylinder be used to predict the ratio of ${ }^{234} \mathrm{U}$ to ${ }^{235} \mathrm{U}$ for that particular cylinder?

Figure 32 through Figure 35 are plots of a potential indicator parameter: mass of ${ }^{232} \mathrm{U}$ divided by the ratio of the mass of ${ }^{234} U$ to the mass of ${ }^{235} U$ (i.e., 232/(234/235)). Ideally, this indicator ratio would be constant over the entire range of fuels, so that regardless of the fuel's initial enrichment, specifics of the fuel's time in the reactor and the time since being extracted from the reactor, ${ }^{232} \mathrm{U}$ concentration would be proportional to 234/235. As these figures show, the behavior of this indicator ratio is far from constant, and varies by more than an order of magnitude over this fuel parameter range. Figure 25 and Figure 30 partially reveal the explanation for this behavior: The production and loss mechanisms for ${ }^{232} \mathrm{U}$ and ${ }^{234} \mathrm{U}$ are very different and consequently, their behaviors relative to the ${ }^{235} \mathrm{U}$ are very different as well. 


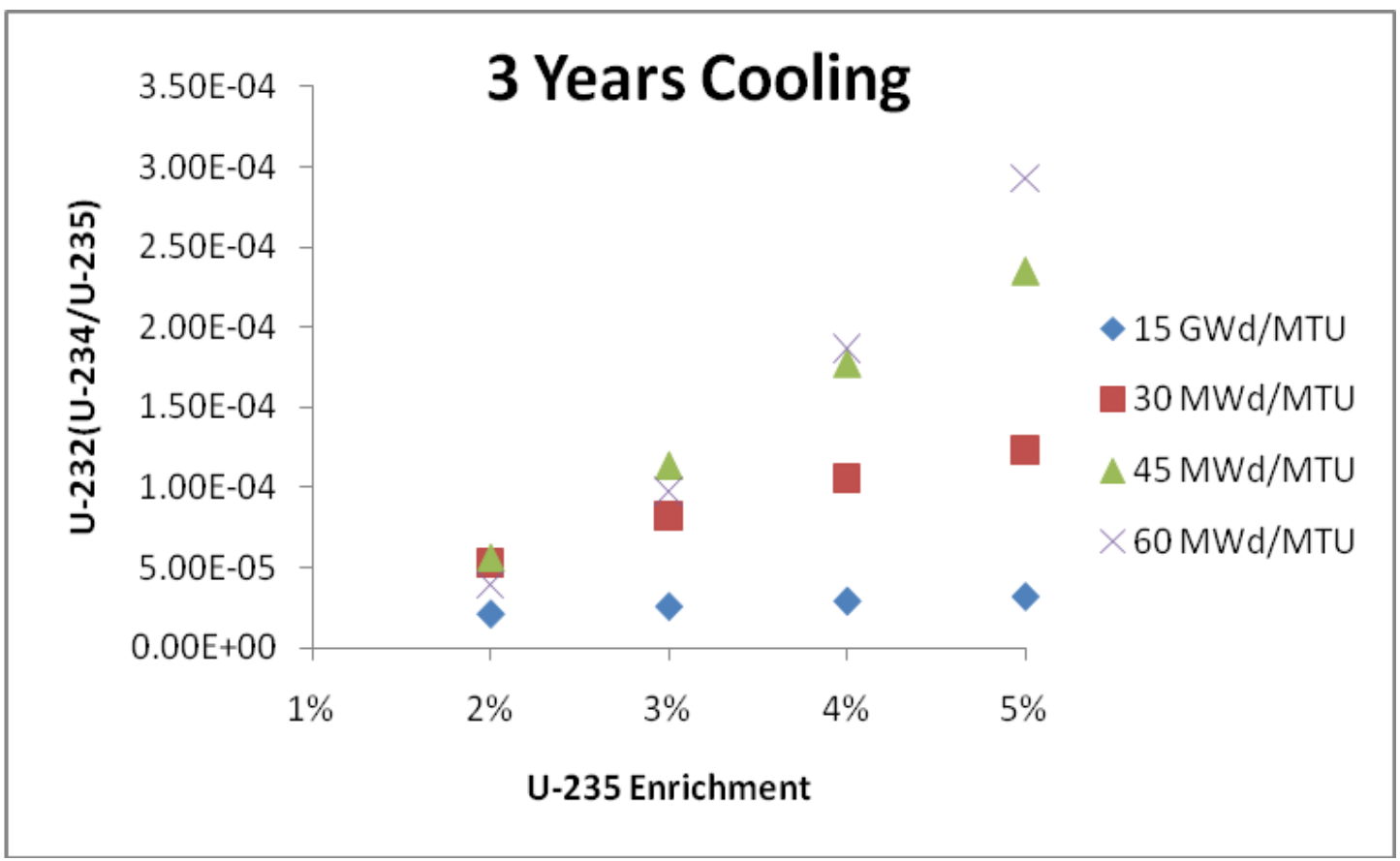

Figure 32. Uranium-232/( $\left.{ }^{234} \mathrm{U} /{ }^{235} \mathrm{U}\right)$ for a cooling time of 3 years, as a function of initial enrichment and burnup

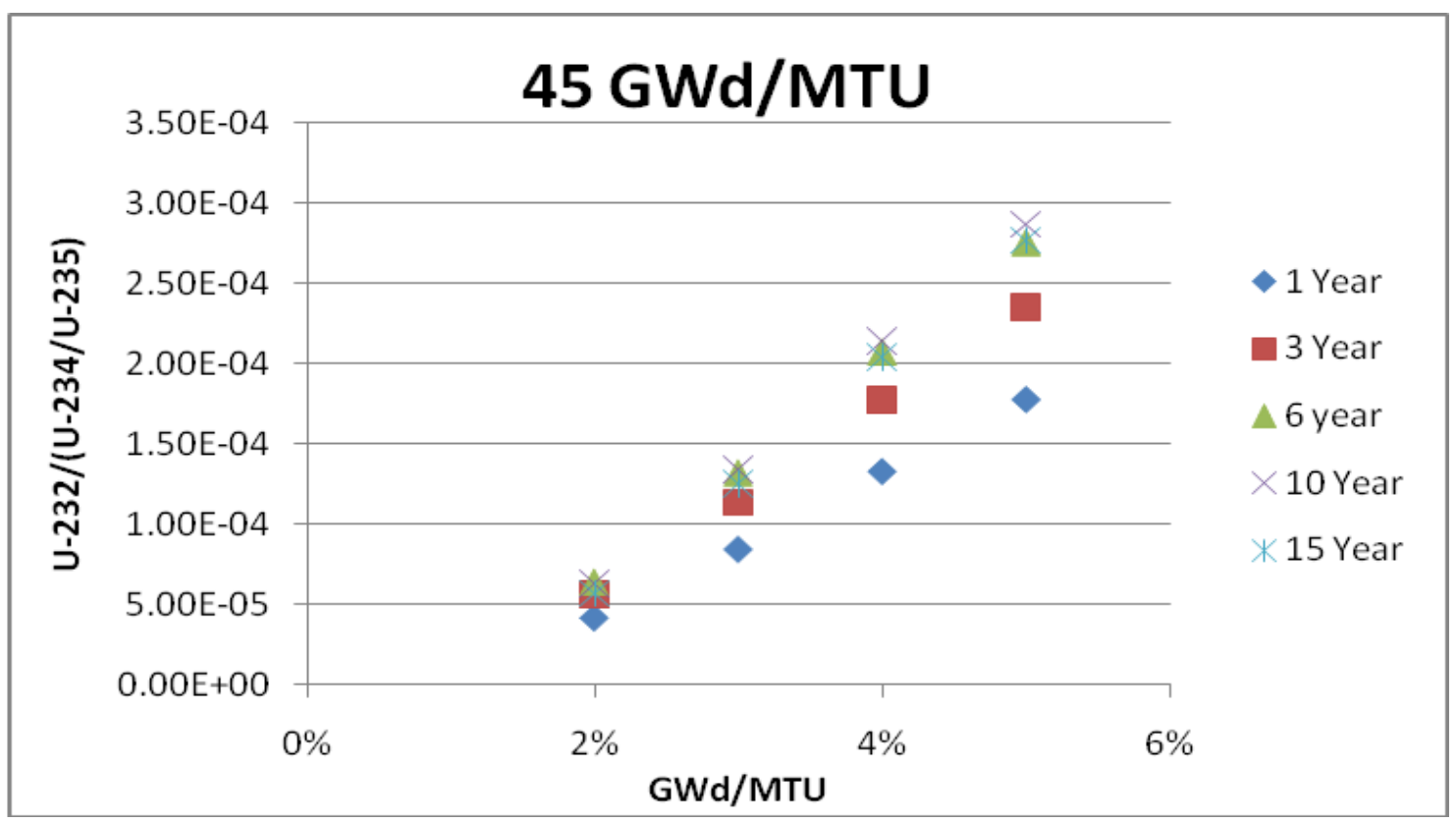

Figure 33. Uranium-232/( $\left.{ }^{234} \mathrm{U} /{ }^{235} \mathrm{U}\right)$ for a burnup of $45 \mathrm{GWd} / \mathrm{MTU}$, as a function of initial enrichment and burnup 


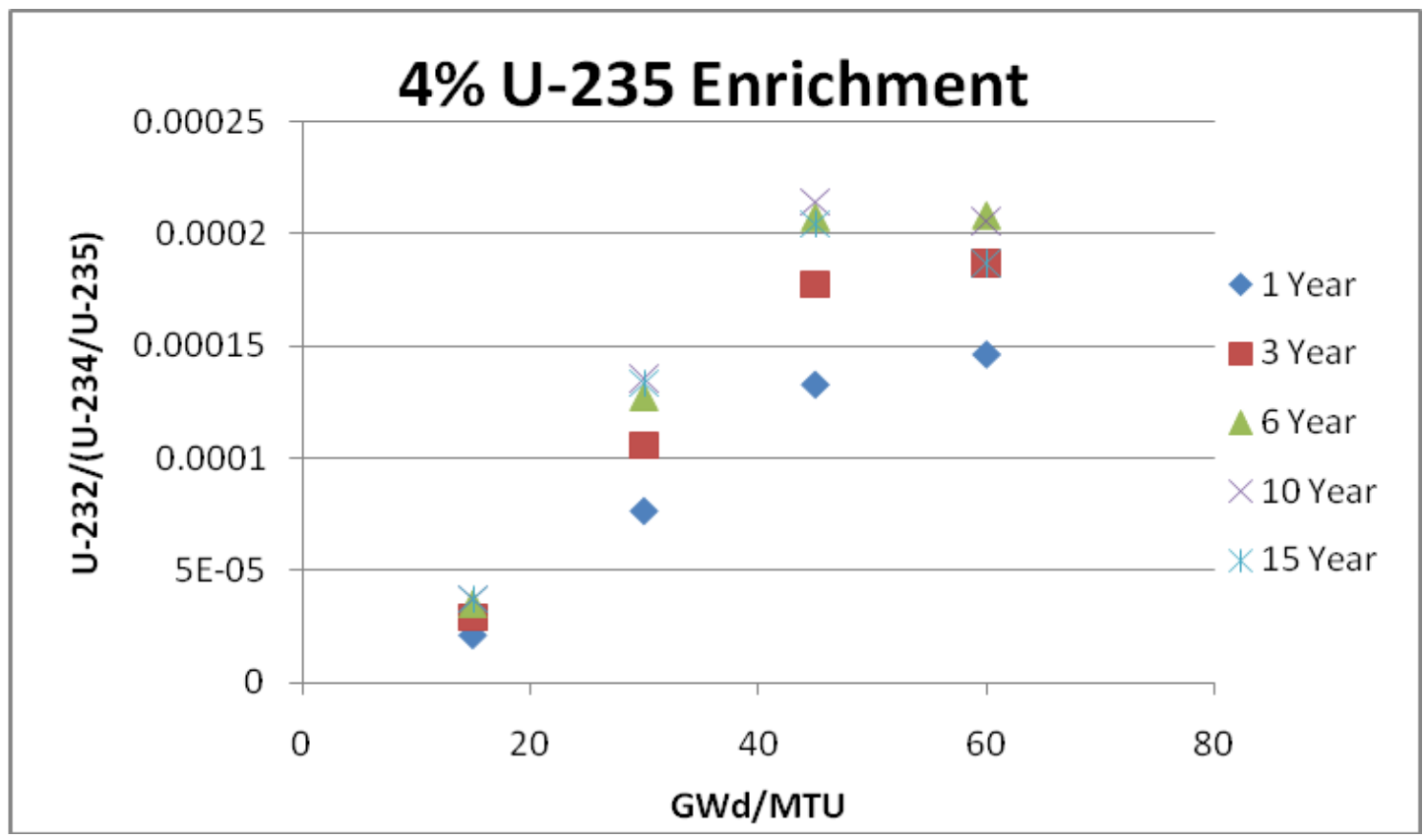

Figure 34. Uranium-232/ $\left({ }^{234} \mathrm{U} /{ }^{235} \mathrm{U}\right)$ for an initial enrichment of $4 \mathrm{wt} \%{ }^{235} \mathrm{U}$, as a function of initial enrichment and burnup

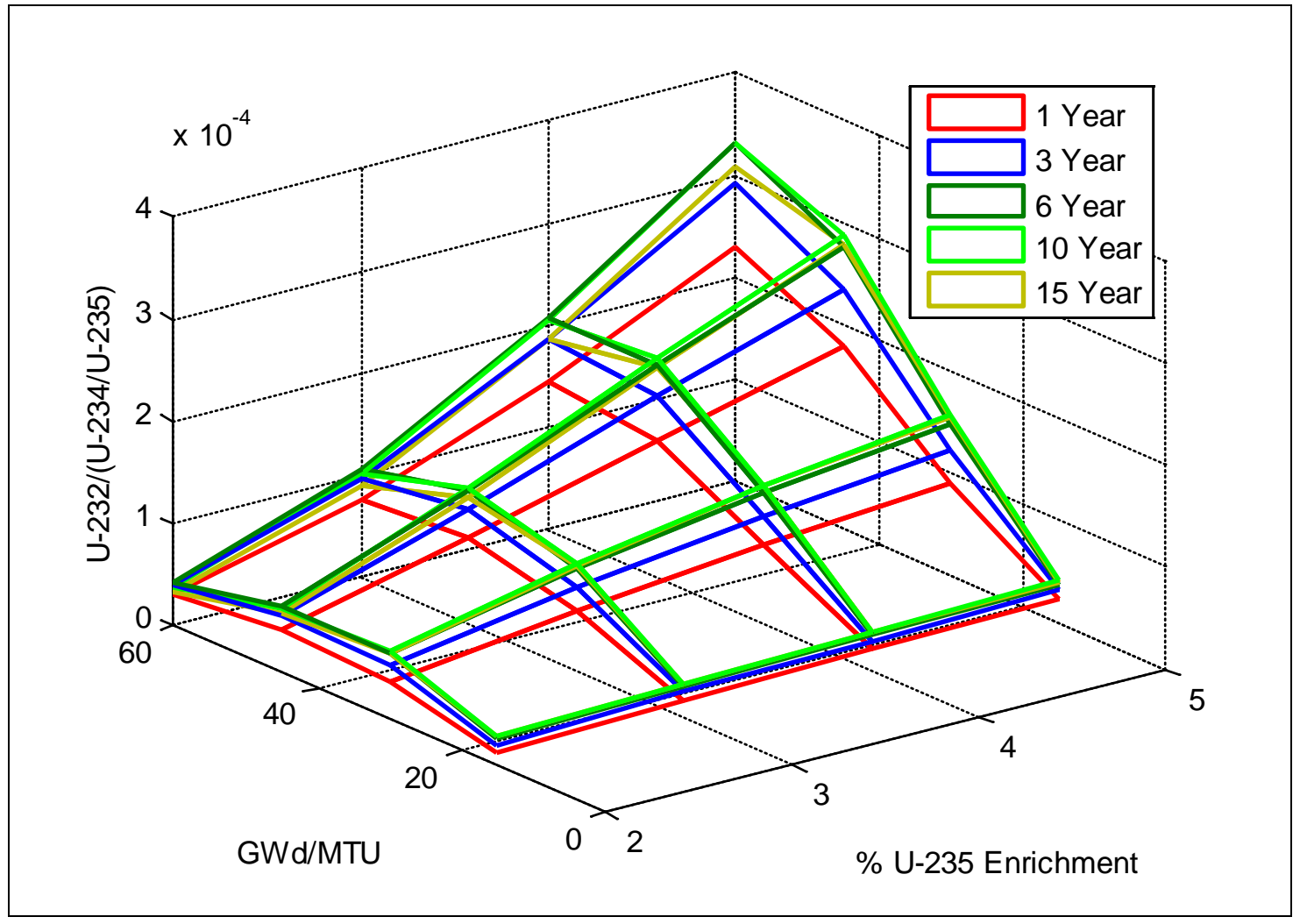

Figure 35. Plot of ${ }^{232} \mathrm{U} /\left({ }^{234} \mathrm{U} /{ }^{235} \mathrm{U}\right)$ ratio for all burnup levels, cooling times and ${ }^{235} \mathrm{U}$ enrichments 
Figure 36 shows additional data on the trends in ${ }^{232} U$ mass, focusing only on the cooling time of the fuel after the last cycle of irradiation. The mass of ${ }^{232} U$ (half life 69.8 years) initially grows within the spent fuel due to the decay of ${ }^{236} \mathrm{Pu}$ (half life 2.87 years) and then levels off as the two isotopes come into secular equilibrium. It should be noted that the calculations in this work are focused entirely on the uranium isotopics of spent fuel prior to reprocessing and that the minor isotope behavior will change after reprocessing. For example, once the ${ }^{236} \mathrm{Pu}$ is removed during reprocessing, it is no longer a source for additional ${ }^{232} \mathrm{U}$ production and the ${ }^{232} \mathrm{U}$ concentration will depend primarily on its half-life.

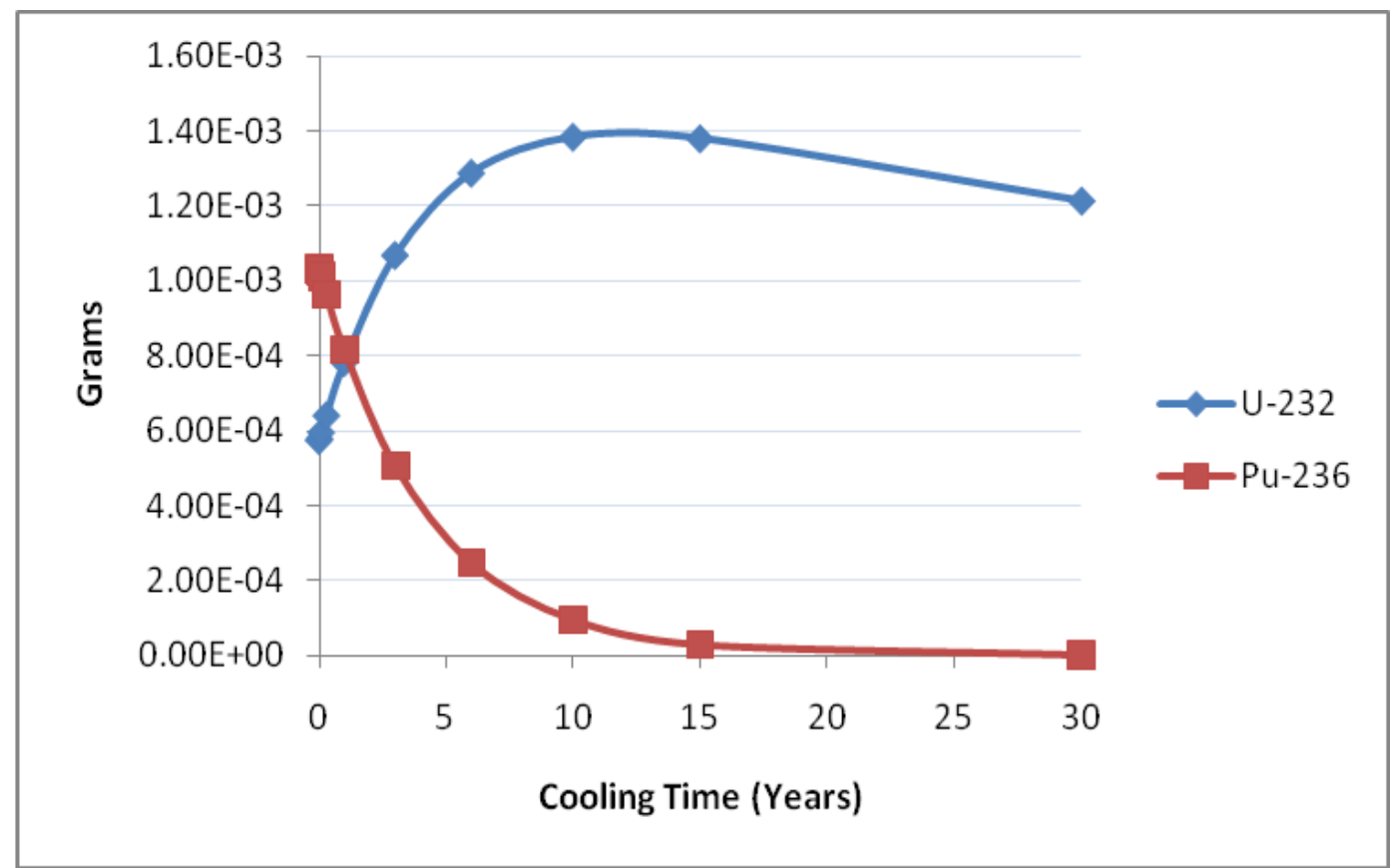

Figure 36. ORIGEN-ARP data for the behavior of ${ }^{232} \mathrm{U}$ and ${ }^{236} \mathrm{Pu}$ in spent fuel based on 1 metric ton of $4 \%{ }^{235} \mathrm{U}$ enriched uranium and $45 \mathrm{GWD} / \mathrm{MTU}$ burnup

\subsection{Preliminary Findings: Non-Traditional Signature for Reactor- Recycle Uranium}

The results presented here indicate that the variability of 234/235 in reactor recycle uranium is significant, up to over 30 times the value for natural uranium. This variability, in and of itself, does not preclude the use of the non-traditional signature (high-energy capture gamma rays from ${ }^{234} \mathrm{U}$-spawned neutrons) for the accurate assay of reactor-recycle uranium, if another signature can be found to unambiguously calibrate that 234/235 behavior. Uranium-232, a minor isotope produced during uranium irradiation in a reactor, is a prime candidate to provide that signature because of a prominent and relatively penetrating $2614-\mathrm{keV}$ gamma-ray emitted by its daughter ${ }^{208} \mathrm{Tl}$. Unfortunately, the production and loss mechanisms for ${ }^{232} \mathrm{U}$ and ${ }^{234} \mathrm{U}$ are very different during and after irradiation. The resulting variability in the $232 /(234 / 235)$ precludes the use of ${ }^{232} \mathrm{U}$ to directly calibrate the variability in 234/235 and consequently, neutron-based signatures are unlikely to be effective for the assay of $\mathrm{UF}_{6}$ cylinders derived from reactor-recycle feedstock.

The difficulties of using ${ }^{232} \mathrm{U}$ for $234 / 235$ calibration are likely to increase under more realistic assumptions than those used in this simple scoping study. For example, only the behavior of uranium 
isotopes prior to reprocessing was considered here. Additional perturbations to uranium isotopes are possible during reprocessing, due to the changing contributions of production and loss mechanisms. Also, the nature of today's large-scale reprocessing facilities means that recycled uranium product is likely to be an aggregation from a number of different assemblies, all of which had their own specific initial loading, reactor history and cooling time.

That said, the ${ }^{232} \mathrm{U}$ signature could still be a useful component of a cylinder NDA system based on the hybrid enrichment algorithms. Since the ${ }^{232} \mathrm{U}$ is an unambiguous indicator of reactor-recycle uranium in the cylinder, its presence can be used as a flag to ignore the non-traditional signature in calculating the enrichment value. As discussed previously, the hybrid method is based on a weighted average of the traditional and non-traditional signatures, and the presence of ${ }^{232} \mathrm{U}$ would instruct the algorithm to set the weighting of the non-traditional signature to zero. Further, that same flag would allow IAEA inspectors to quickly determine whether or not the cylinder was derived from the type of feedstock declared by the operator, if that were deemed to be an important parameter in the verification process.

However, the presence of the ${ }^{208} \mathrm{Tl} 2614-\mathrm{keV}$ gamma ray is not assured in product material at enrichment plants. Though further study is necessary, if one assumes that the $\mathrm{UF}_{6}$ used as feed to the enrichment cascade has been recently separated, then the product at withdrawal will contain little contamination from uranium-isotope daughters. If this is the case, detectable amounts of ${ }^{208} \mathrm{Tl}$ would need to grow into the cylinder after it is filled in order to detect the ${ }^{232} \mathrm{U}$ in a verification station measuring the product cylinders. The grow-in time of ${ }^{208} \mathrm{Tl}$ is reasonably well-described by the functional form $1-\exp \left(-\mathrm{a}^{*} \mathrm{t}\right)$, with $\mathrm{a}=0.4 \mathrm{yr}^{-1}$. After 1 year, the ${ }^{208} \mathrm{Tl}$ activity is about one-third of its equilibrium value, increasing to $90 \%$ of its equilibrium value at about 5.8 years. Given this relatively slow grow-in time, the likelihood of detectable ${ }^{208} \mathrm{Tl}$ in product cylinders that are measured within hours, days or even weeks of withdrawal from the cascade may be low (the cylinders measured at the AREVA fuel fabrication plant and depicted in Figure 10 were typically 2-4 months old). In the case of recently separated feed the ${ }^{232} \mathrm{U}$ signatures might be more likely to be detectable in the tails cylinders after sufficient storage time has passed to build up ${ }^{208} \mathrm{Tl}$. However, due to ${ }^{232} \mathrm{U}$ being a lighter isotope, an inadequate amount may remain in the tails to provide a sufficient ${ }^{208} \mathrm{Tl}$ signal for detection. If the feed cylinders have been aged sufficiently after reprocessing but before arrival at the enrichment plant the ${ }^{232} \mathrm{U}$ signatures may be more likely to be detectable. Investigation of the validity of these scenarios and their potential effects on the ${ }^{232} \mathrm{U}$ signal requires additional study. 


\subsection{Future Work}

The results from the FY10 study have encouraged further study, development and refinement of hybrid enrichment assay methods and instrumentation that might ultimately be used in an automated/unattended cylinder verification station. This section summarizes major tasks for that continued development.

Task 1: Refine and Validate Modeling Framework. High-fidelity modeling of the $\mathrm{UF}_{6}$ cylinder scenario is a time- and resource-efficient complement to other development tasks. For example, simulation can quickly explore the impact of various collimator designs (which double as neutron-togamma converters in the hybrid methods), where field study would be time-consuming and expensive. Simulation can also generate large libraries of "virtual assay signatures" to support algorithm development, over the large parameter space important to a viable instrument (e.g., enrichment levels, ${ }^{232} \mathrm{U}$ contamination levels and wall-thickness variation). PNNL developed a functional and preliminarily validated modeling framework in previous work. This tasking will expand, refine and more fully validate the framework (using data from May 2010 measurements of Type 30B cylinders) and will apply those modeling tools to address research questions in other tasks. Of particular interest will be how the spatial distribution of $\mathrm{UF}_{6}$ in the cylinder contributes to the systematic error in cylinders of various types.

Task 2: Perform System Design Parameter Study. There are a number of system design choices to be considered in a hybrid assay method instrument. For example, the design (materials and physical dimensions) of the collimator/converter surrounding the NaI spectrometers can have a marked impact on the intensity of the non-traditional signature. Modeling studies will explore different collimation/conversion designs that recognize the energy distribution of the neutrons escaping the $\mathrm{UF}_{6}$ volume and the energy-dependent cross-section for neutron capture on ${ }^{56} \mathrm{Fe}$ (the dominant component of the non-traditional high-energy gamma signature). Other design parameters to consider include the size, shape and orientation of the NaI spectrometers and the shape and materials to be used in the reflector material used to reflect neutrons back toward the steel volume of the $\mathrm{UF}_{6}$ cylinder. These studies will investigate the balance between the performance of the traditional assay method (which benefits from a high degree of collimation and small detector area) and the non-traditional method (which benefits from less collimation and larger detectors).

Task 3: Refine and Evaluate Traditional Method using Square-Wave Convolute Algorithm. The Square Wave Convolute (SWC) algorithm developed and implemented in the FY10 study, though firstgeneration, effectively extracted the net count rate in the $186-\mathrm{keV}$ peak by removing both constant and linear components of the underlying continuum - a large source of uncertainty in the traditional enrichment-meter approach. Testing indicated that the SWC algorithm will also be relatively insensitive to variations in energy resolution and gain drift in an unattended monitoring scenario. However, the current SWC algorithm performs relatively poorly at low enrichments (i.e., natural and depleted) where the background is pronounced relative to the 186-keV peak, and the impact of curvature in the background shape (which are not removed by a symmetric digital filter) is most acute. We will investigate and test methods for overcoming these challenges using measured and simulated data sets. We will then compare the SWC algorithm to other codes (e.g., NaIGEM [Gunnink 2001]) that implement direct fitting methods to assess the relative strengths and weaknesses in terms of assay accuracy and practical operational issues such as robustness against gain and resolution instabilities. 
Task 4: Refine and Evaluate Hybrid Algorithm. The FY10 scoping study used a simple average of the traditional and non-traditional signature, over all enrichments from depleted to $5 \mathrm{wt} \%{ }^{235} \mathrm{U}$. More sophisticated weighting functions for the traditional and non-traditional signatures need study. For example, it may be beneficial to place a greater weight on the non-traditional signature for tail and feed cylinders. Conversely, the traditional signature may be weighted more heavily at higher enrichments where the uncertainty in the $186-\mathrm{keV}$ peak count rate is low and changes in the ${ }^{234} \mathrm{U} /{ }^{235} \mathrm{U}$ ratio (e.g., from reactor recycle material) may lead to systematic variations in the non-traditional signature. Because the hybrid methods are based on full-spectrum ( $50 \mathrm{keV}$ to $9 \mathrm{MeV}$ ) data acquisition, there also exists the possibility of mining additional spectral regions for other information useful to cylinder verification not discussed in this work (e.g., wall-thickness estimation, rapid detection of ${ }^{232} \mathrm{U}$ for indication of reactorrecycle feedstock).

Task 5: Develop Field Prototypes and Perform Field Measurements with Graded Approach. The FY10 study indicated that a hybrid assay method can meet target uncertainties for depleted and natural enrichments, but that analysis was based on very few Type 30B cylinders, rather than the Type 48 cylinders that are the industry standard for feed and tail material. Definitive assessment of the viability of the hybrid NDA methods for product, tail and feed cylinders will require an extended measurement campaign at an operating enrichment plant. Such a campaign should utilize an array of NaI spectrometers and an accompanying suite of collimator/converters, pulse-processing electronics and enrichment analysis algorithms specifically tailored to the hybrid NDA methods. We will explore the possibility of such a campaign in collaboration with ORNL and LANL, possibly through the EURATOM PCG.

In keeping with a graded approach to instrument development and testing, however, PNNL will pursue the possibility of continuing to use the AREVA fuel fabrication plant in Richland, Washington facility as a convenient and cost-effective location for testing and evaluation of both hardware design options and algorithm variants. AREVA receives new shipments of Type-30B cylinders weekly, with a range of enrichments from depleted (0.2\%) to 5\% and we may be able to measure upwards of 200 Type-B cylinders in a few months. Importantly, the AREVA cylinder population comes from a variety of enrichment providers (e.g., Urenco, USEC, Ural) so that we may more fully investigate some of the systematic sources of uncertainty that might arise from different enrichment processes. While previous measurements at AREVA have used a mobile platform and one-day campaigns, we will explore the possibility of a semi-permanent installation in the existing AREVA cylinder NDA area. To minimize the impact on AREVA operations, we may be able to install the PNNL hybrid instrumentation opposite the AREVA HPGe system and perform cylinder measurements with the two NDA systems simultaneously.

Task 6: Study Minor Isotope Variations. The utility of the non-traditional signatures for ${ }^{235} \mathrm{U}$ enrichment quantification depends on the stability of ${ }^{234} \mathrm{U} /{ }^{235} \mathrm{U}$ ratio as a function of feed type, process type (e.g., diffusion or centrifuge) and process variables. Previous work indicates that the ratio will be relatively stable for ore-based feed and a facility-specific calibration, but PNNL's FY10 modeling and analysis of reactor-recycle uranium indicates that the ${ }^{234} \mathrm{U} /{ }^{235} \mathrm{U}$ ratio in reactor recycle feedstock varies in ways that preclude using the non-traditional signature as a reliable metric for ${ }^{235} \mathrm{U}$ enrichment. Better understanding of the potential (and shortcomings) of ${ }^{234} U$ signatures will necessitate further exploration of the ${ }^{234} \mathrm{U} /{ }^{235} \mathrm{U}$ ratio. In this task, we will survey the literature and communicate with technical staff and organizations that could provide data and information regarding the variation in minor isotopes ${ }^{232} U,{ }^{234} U$ and ${ }^{236} U$ ) in industrial enrichment settings. We will also explore the possibility of utilizing data from IAEA's routine environmental sampling activities at enrichment plants to provide useful calibration and verification of the behavior of the minor isotope ratios (particularly ${ }^{234} \mathrm{U} /{ }^{235} \mathrm{U}$ ) at each specific facility. 
We should also consider other feedstock variants, for example tails recycle, and those lessons incorporated into discussions about the potential of the hybrid methods for automated cylinder verification. 
PNNL-19854

\subsection{References}

Aigner, H, et al. 2001. International Target Values 2000 for Measurement Uncertainties in Safeguarding Nuclear Materials. Report, International Atomic Energy Agency, Vienna, Austria.

American National Standards Institute. 1995. "Packaging of $\mathrm{UF}_{6}$ for Transport." Accessed at American National Standards Institute, New York.

Berndt, R, E Franke, and P Mortreau. 2010. "U-235 Enrichment or UF6 Mass Determination on UF 6 Cylinders with Non-Destructive Analysis Methods." Nuclear Instruments \& Methods in Physics Research Section A-Accelerators Spectrometers Detectors and Associated Equipment 612(2):30919. 10.1016/j.nima.2009.10.060.

Ciemala, M, et al. 2009. "Measurements of High-Energy [Gamma]-Rays with LaBr3:Ce Detectors." Nuclear Instruments and Methods in Physics Research Section A: Accelerators, Spectrometers, Detectors and Associated Equipment 608(1):76-79.

Cochran, RG, and N Tsoulfanidis. 1990. The Nuclear Fuel Cycle: Analysis and Management. 2nd ed., American Nuclear Society, La Grange Park, Illinois.

Cooley, J, et al. 2000. "Experience with Environmental Swipe Sampling in a Newly Built Gas Centrifuge Enrichment Plant." in Proceedings of the 44th Annual Meeting of the International Nuclear Materials Management (INMM), Accessed at INMM.

Croff, AG. 1983. "Origen2: A Versatile Computer Code for Calculating the Nuclide Compositions and Characteristics of Nuclear Materials." Nuclear Technology 62(3).

Curtis, MM. In Press. "Supplemental Systems for Unattended UF 6 Cylinder Monitoring." in Institute of Nuclear Materials Management 51st Annual Meeting, Vol PNNL-SA-73233, Accessed at https://erica.pnl.gov/ir/upload/prod_rvw.asp?product_id=291692. INMM, Baltimore, MD.

Dias, F, and et al. 2008. "Verification of U-235 Isotopic Abundance in Low Enriched UF6 Cylinders by a Low Resolution Spectroscopy Technique." in Proceedings of the 49th Annual Meeting of the Institute of Nuclear Materials Management (INMM), Accessed at INMM.

Fischer, D, et al. 2005. "Environmental Sampling for Detecting Undeclared Nuclear Materials/Activities." in Changing the Safeguards Culture: Broader Perspectives and Challenges: Proceedings of the 5th Joint INMM/ESARDA Workshop, pp. 2.7. Accessed at Institute of Nuclear Materials Management; European Safeguards Research and Development Association, Santa Fe, NM.

Gunnink, R, and R Arlt. 2001. "Methods for Evaluating and Analyzing Cdte and Cdznte Spectra." Nuclear Instruments \& Methods in Physics Research Section A-Accelerators Spectrometers Detectors and Associated Equipment 458(1-2):196-205. 
Janssens, F, and JP Francois. 1991. "Evaluation of 3 Zero-Area Digital-Filters for Peak Recognition and Interference Detection in Automated Spectral Data-Analysis." Analytical Chemistry 63(4):32031.

Knoll, GF. 2000. Radiation Detection and Measurement. 3rd ed., Wiley, New York.

Menlove, H. In Press. "Materials Control and Accountability: Neutron Detector Materials and Technology: Alternatives to Helium-3." in Institute of Nuclear Materials Management 51st Annual Meeting, Accessed at INMM, Baltimore, Maryland.

Moszynski, M, et al. 2002. "Intrinsic Energy Resolution of NaI(Tl)." Nuclear Instruments and Methods in Physics Research Section A: Accelerators, Spectrometers, Detectors and Associated Equipment 484(1-3):259-69.

Nicolini, R, et al. 2007. "Investigation of the Properties of a 1"×1" LaBr3:Ce Scintillator." Nuclear Instruments and Methods in Physics Research Section A: Accelerators, Spectrometers, Detectors and Associated Equipment 582(2):554-61.

Op de Beeck, J. 1975. "Gamma-Ray Spectrometry Data-Collection and Reduction by Simple Computing Systems." Atomic Energy Review 13(4):743-805.

Peurrung, A. 1999. "Predicting ${ }^{232}$ U Content in Uranium." pp. 22. Accessed at http://www.osti.gov/bridge/servlets/purl/2657-gnDcoJ/webviewable/. Pacific Northwest National Laboratory, Richland, Washington.

Quarati, F, et al. 2007. "X-Ray and Gamma-Ray Response of a 2"×2" LaBr3:Ce Scintillation Detector." Nuclear Instruments and Methods in Physics Research Section A: Accelerators, Spectrometers, Detectors and Associated Equipment 574(1):115-20.

Reilly, D, et al. 1991. Passive Nondestructive Assay of Nuclear Materials. Report, US Nuclear Regulatory Comission, Washington DC.

Reilly, TD, et al. 1974. "Continuous in-Line Monitor for UF 6 Enrichment." Nuclear Technology 23(3):318-27.

Richter, S, et al. 1999. "Isotopic "Fingerprints" For Natural Uranium Ore Samples." International Journal of Mass Spectrometry 193(1):9-14.

Smith, LE, et al. 2010. Hybrid Enrichment Assay Methods for a $\mathrm{UF}_{6}$ Cylinder Verification Station. Report, Pacific Northwest National Laboratory, Richland, WA.

Smith, LE, EK Mace, and AC Misner. In press. "Signatures and Methods for the Automated Nondestructive Assay of UF6 Cylinders at Uranium Enrichment Plants." IEEE Transactions on Nuclear Science. 
Smith, LE, et al. 2009. "Development of a Portal Monitor for $\mathrm{UF}_{6}$ Cylinder Verification." in INMM 50th Annual Meeting, Accessed at INMM, Tuscon, AZ.

Stromswold, DC, et al. 2005. Highly Enriched Uranium Detection Via ${ }^{232}$ U -- Summary Report, PNNL15352. Report, Pacific Northwest National Laboratory, Richland, WA.

Walton, RB, et al. 1974. "Measurements of $\mathrm{UF}_{6}$ Cylinders with Portable Instruments." Nuclear Technology 21(2):133-48.

Wilson, WB, et al. 2002. SOURCES 4C: A Code for Calculating $(\alpha, N)$, Spontaneous Fission, and Delayed Neutron Sources and Spectra. Report, Los Alamos National Laboratory, Los Alamos. 



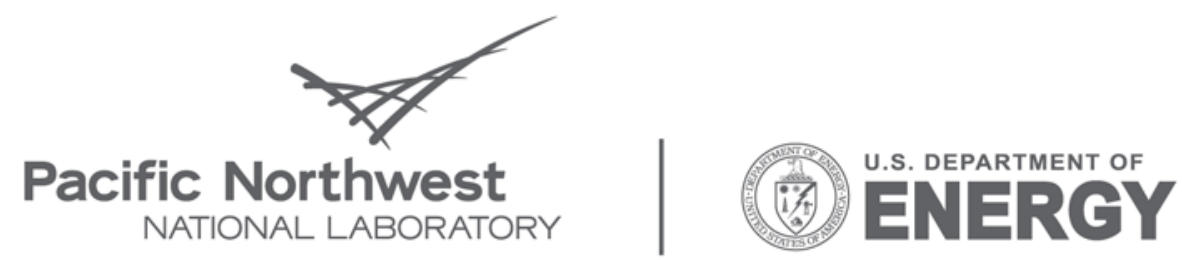

Proudly Operated by Battelle Since 1965

902 Battelle Boulevard

P.O. Box 999

Richland, WA 99352

1-888-375-PNNL (7665)

www.pnl.gov 